\title{
ESTUDO DAS DESCRIÇÕES LAGRANGIANA E EULERIANA NA ANÁLISE NÃO-LINEAR GEOMÉTRICA COM O EMPREGO DO MÉTODO DOS ELEMENTOS FINITOS
}

Cristina Ferreira de Paula

Dissertação apresentada à Escola de Engenharia de São Carlos da Universidade de São Paulo, como parte dos requisitos para obtenção do título de Mestre em Engenharia de Estruturas.

Orientador: Prof. Dr. Sergio Persival Baroncini Proença

São Carlos 
Ficha catalográfica preparada pela Seçăo de Tratamento da Informação do Serviço de Biblioteca - EESC-USP

Paula, Cristina Ferreira de

P324e Estudo das descrições Lagrangiana e Euleriana na análise não-linear geométrica com emprego do método dos elementos finitos / Cristina Ferreira de Paula. -- São Carlos, 1997.

Dissertação (Mestrado). -- Escola de Engenharia de São Carlos-Universidade de São Paulo, 1997. Área: Engenharia de Estruturas Orientador: Prof. Dr. Sergio Persival Baroncini Proença

1. Método dos elementos finitos. 2. Nãolineraridade geométrica. 3. Princípio dos trabalhos virtuais. 4. Estabilidade do equilíbrio. I. Título 
Aos meus pais Francisco e Guilhermina que sempre souberam qual o caminho apontar-me. 
'O sucesso de um homem está nas mãos do Senhor, é ele que dá ao escriba a sua glória'.

Eclo 10,5 


\section{AGRADECIMENTOS}

Ao Prof. Dr. Sérgio P.B. Proença pela excelente orientação, amizade e incentivo durante a realização deste trabalho.

Ao Prof. Barreiro pela amizade e colaboração.

Aos meus pais, irmãos e familiares pelo carinho e apoio em todos os momentos.

À minha comunidade, um tesouro concedido por Deus.

Aos verdadeiros amigos pela sincera amizade.

Aos colegas do Departamento de Estruturas da Escola de Engenharia de São Carlos.

Aos amigos Leila, Rivelli, Patrícia e Daniel pelo carinho e apoio durante estes anos.

À Universidade Federal de Ouro Preto, pela formação acadêmica.

À Maria Nadir Minatel, pelo auxilio no trabalho de referência bibliográfica.

A todas as pessoas que direta ou indiretamente contribuíram para a realização deste trabalho.

À CAPES e à FAPESP, pelo apoio financeiro.

E acima de tudo, a Deus por mais esta oportunidade. 


\section{ÍNDICE}

LISTA DE FIGURAS …....................................................................... i

LISTA DE TABELAS ......................................................................... iv

LISTA DE SÍMBOLOS ..................................................................

ABREVIATURAS …................................................................................. xii

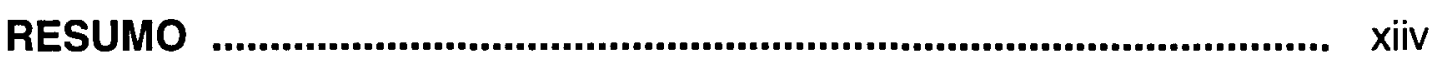

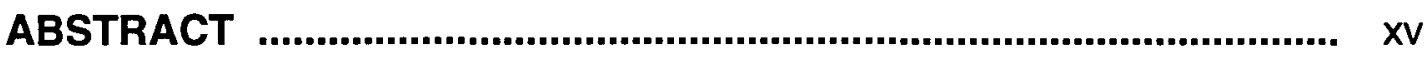

CAPÍTULO 1- INTRODUÇÃO …....................................................... 1

CAPÍTULO 2- ASPECTOS DA MECÂNICA DO CONTÍNUO ................. 4

2.1 Descrição material e espacial do movimento ............................ 4

2.2 Tensores de deformação ........................................................ 5

2.3 Tensores de tensão ............................................................. 7

2.4 Princípio dos Trabalhos Virtuais ................................................ 11

CAPÍTULO 3- APLICAÇÃO DO MÉTODO DOS ELEMENTOS FINITOS 15

3.1 Equações de equilíbrio discretizadas ..................................... 15

3.2 Equações incrementais de equilíbrio ......................................... 17

3.3 Matriz de rigidez tangente ..................................................... 18

3.4 Formulações Lagrangiana Total e Atualizada em elementos finitos 
3.4.1 Formulação Lagrangiana Total ..................................... 19

3.4.1.1 Elemento finito de treliça plana .................................. 19

3.4.1.2 Elemento finito de pórtico plano .................................. 25

3.4.1.2.1 Aproximação na matriz de rigidez tangente .. 29

3.4.1.3 Elemento finito para análise bidimensional ................. 36

3.4.2 Formulação Lagrangiana Atualizada .......................... 41

3.4.2.1 Elemento finito de treliça plana ................................... 43

3.4.2.2 Elemento finito de pórtico plano ................................. 46

3.4.2.3 Elemento finito para análise bidimensional ................ 49

CAPÍTULO 4- ANÁLISE DA ESTABILIDADE DO EQUILÍBRIO ........... 52

4.1 Introdução à instabilidade .................................................... 52

4.2 Estudo dos algoritmos para caracterização de pontos singulares e do tipo de instabilidade ....................................... 60

4.3 Método incremental iterativo de Newton-Raphson .................. 61

4.4 Métodos de continuação ......................................................... 64

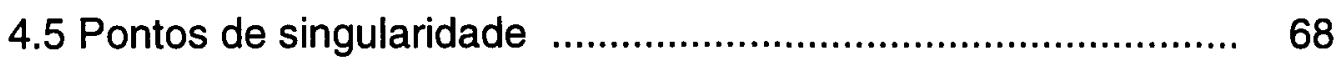

4.5.1 Definição do critério matemático para caracterização de pontos limite e de bifurcação ..................................... 68

4.5.2 Critério para caracterização dos pontos singulares na análise estrutural ..................................................... 77

4.6 Formulação de sistema estendido ........................................... 78

4.7 Método de Newton para solução de sistemas estendidos ....... 82

4.8 Algoritmo geral para consideração da estabilidade em problemas não-lineares ...................................................... 86

5.1 Exemplo de NLG em treliça espacial ...................................... 91

5.2 Exemplo de carga limite - treliça simples ................................. 93

5.3 Exemplo de carga crítica - barra comprimida axialmente ........ 95 
CAPÍTULO 6- CONSIDERAÇÕES FINAIS E CONCLUSÕES

REFERÊNCIAS BIBLIOGRÁFICAS

ANEXO A 


\section{LISTA DE FIGURAS}

FIGURA 2.1- Partícula em movimento ................................................... 4

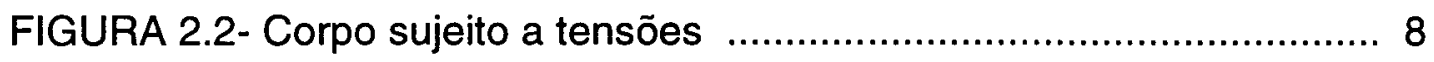

FIGURA 2.3- Barra axialmente tracionada ............................................ 10

FIGURA 2.4- Princípio dos trabalhos virtuais para um sólido .................. 12

FIGURA 3.1- Elemento finito de treliça plana .......................................... 20

FIGURA 3.2- Elemento finito de pórtico plano ........................................ 25

FIGURA 3.3- Campo de deslocamentos na flexão .................................. 26

FIGURA 3.4- Elemento finito bidimensional em coordenadas adimensionais ...................................................... 37

FIGURA 3.5- Atualização da configuração de referência .......................... 42

FIGURA 4.1- Trajetórias de bifurcação de equilíbrio e comportamento pósflambagem .................................................................... 53

FIGURA 4.2- Treliça simples ……...................................................... 55

FIGURA 4.3- Comportamento não-linear de uma treliça simples ............. 57

FIGURA 4.4- Curva carga/deslocamento de uma treliça simples .............. 59 
FIGURA 4.5- Curva carga/deslocamento de um cabo 59

FIGURA 4.6- Método de Newton-Raphson 63

FIGURA 4.7a- Método de comprimento de arco com iteração em um 'plano normal' 66

FIGURA 4.7b- Método de comprimento de arco com iteração em uma 'esfera' de raio ds 67

FIGURA 4.8a- Ponto limite 69

FIGURA 4.8b- Bifurcação em ponto de dobra 70

FIGURA 4.8c- Bifurcação em ponto limite 70

FIGURA 4.9- Tipos de bifurcação 75

FIGURA 4.10- Inspeção do det $K_{T}$ 87

FIGURA 5.1- Treliça espacial de base quadrada 92

FIGURA 5.2- Deslocamento horizontal do ponto 9 93

FIGURA 5.3- Treliça simples 94

FIGURA 5.4- Deslocamento vertical do ponto B 95

FIGURA 5.5- Barra sujeita à compressão centrada 96

FIGURA 5.6- Comportamento não-linear geométrico de um barra sujeita à compressão centrada 98

FIGURA A.1- Espaços de Banach 112 
FIGURA A.2- Posição inicial e deslocada de três pontos próximos 114

FIGURA A.3- Diferenciais de tensão em equilíbrio 116 


\section{LISTA DE TABELAS}

Tabela 4.1- Exemplos de equações de restrição ..................................66 66 


\section{LISTA DE SÍMBOLOS}

$a_{i j k}, b_{i j}, c_{i}=$ constantes da equação de bifurcação

$A_{0}=$ área na configuração indeformada

$A=$ área na configuração deformada

$B, B_{0}, B_{L}, C, G, H=$ matrizes auxiliares

$b_{0}=$ forças por unidade de volume na configuração inicial

$\dot{b}_{0}=$ taxa da força por unidade de volume na configuração inicial

$b=$ forças por unidade de volume na configuração atual

$\dot{b}=$ taxa da força por unidade de volume na configuração atual

$\mathrm{D}=$ matriz constitutiva do material

$d A_{\circ}=$ diferencial de área na configuração indeformada

$\mathrm{d} A=$ diferencial de área na configuração deformada

$\operatorname{det} J_{c}=$ determinante da transformação Jacobiana

$d \hat{P}=$ força na configuração indeformada (inicial) 
$d P=$ força na configuração deformada (atual)
$d x=$ comprimento na configuração deformada
$d X=$ comprimento na configuração indeformada

$\mathrm{dVo}=$ diferencial de volume na configuração indeformada

$\mathrm{dV}=$ diferencial de volume na configuração deformada

$E=$ módulo elástico de rigidez

$\mathrm{El}=$ produto do módulo elástico de rigidez pelo momento de inércia

$f=$ função de restrição

$F=$ tensor gradiente de deformação

$G(q)=$ função que representa o sistema de equações não-lineares

grad $u=$ gradiente dos deslocamentos na configuração atual

$\mathrm{h}=$ altura da treliça com relação à horizontal

$H^{*}, B^{*}, C^{*}, G^{*}=$ matrizes auxiliares na formulação Lagrangiana Atualizada

$\mathrm{J}=$ determinante do tensor gradiente de deformação

$J_{c}=$ matriz da transformação Jacobiana

$\mathrm{K}=$ rigidez da mola

$K_{i}=$ parcela $\mathrm{i}$ da matriz de rigidez tangente para o elemento finito de pórtico plano

$\mathrm{K}_{\mathrm{L}}=$ matriz de rigidez de correção das coordenadas 
$\mathrm{K}_{\mathrm{o}}=$ matriz de rigidez elástica linear

$\mathrm{K}_{\sigma}=$ matriz de rigidez geométrica

$\mathrm{K}_{\mathrm{T}}=$ matriz de rigidez tangente

$\mathrm{K}_{\mathrm{T} \theta}=$ matriz de rigidez tangente para o elemento finito bidimensional

$\mathrm{L}=$ comprimento atual

$\mathrm{L}_{\mathrm{o}}=$ comprimento inicial

$M=$ matriz das funções interpoladoras para o elemento finito de treliça plana

$\mathrm{N}=$ força normal

$\mathrm{n}=$ versor normal à superfície indeformada

$N(T)=$ núcleo do operador $T$

$N\left(T^{*}\right)=$ núcleo do operador dual $T^{*}$

$N_{i}=$ funções interpoladoras

$N_{o}=$ força normal inicial

$\mathrm{P}=$ carga genérica aplicada

$\Pi=$ tensor de tensão de Piola-Kirchhoff de $1^{\underline{a}}$ espécie

$q=$ vetor dos deslocamentos nodais

$q_{i}=$ deslocamento nodal correspondente ao grau de liberdade $i$

$r=$ resíduo 
$\mathrm{R}=$ tensor de rotação

$R(T)=$ imagem da transformação linear $T$

$R\left(T^{*}\right)=$ imagem da transformação linear dual $T^{*}$

$\mathrm{S}=$ tensor de tensão de Piola-Kirchhoff de $2^{\mathrm{a}}$ espécie na configuração inicial

$T=$ operador linear

$\mathrm{t}=$ tempo

$T^{\star}=$ operador linear dual

$\overline{\mathrm{t}}=$ força por unidade de área na configuração atual

$\overline{\mathrm{t}}_{\mathrm{o}}=$ força por unidade de área na configuração inicial

$\dot{\overline{\mathrm{t}}}=$ taxa da força por unidade de área na configuração atual

$\dot{\bar{t}}_{\mathrm{o}}=$ taxa da força por unidade de área na configuração inicial

$u^{\prime}=$ derivada do deslocamento axial com relação $\mathrm{a} X$ ou $\mathrm{X}$

$u=$ função deslocamento axial da barra

$\mathrm{U}=$ tensor relacionado à deíormação

$\mathrm{u}^{*}=$ acréscimo no comprimento após deformação

$u_{e}=$ função de deslocamentos para o elemento finito bidimensional

$u_{o}=$ deslocamento axial dos pontos do eixo da barra

$u(X, Y)=$ função do deslocamento na direção do eixo da barra 


$$
\begin{gathered}
u_{\alpha, \beta}=\text { derivadas parciais das componentes do deslocamento para } 0 \\
\text { elemento finito bidimensional }
\end{gathered}
$$

$\dot{\mathrm{u}}=$ derivada do deslocamento em relação ao tempo (velocidade)

$v(X, Y)=$ função do deslocamento transversal ao eixo da barra

$v_{0}=$ deslocamento transversal dos pontos do eixo da barra

$V^{\prime}=$ derivada do deslocamento transversal com relação a $X$ ou $X$

$V_{0}=$ volume na configuração inicial

$V=$ volume na configuração atual

$x=$ coordenada na configuração deformada

$X=$ coordenada na configuração inicial

$X_{e}=$ coordenadas iniciais para o elemento finito bidimensional

$\theta=$ ângulo da barra com a vertical

$\phi_{i}^{*}=$ conjunto de vetores linearmente independentes do espaço $X^{*}$

$\phi_{i}=$ conjunto dos versores linearmente independentes que forma o $N\left(G_{u}^{\circ}\right)$

$\psi_{i}^{*}=$ conjunto dos versores linearmente independentes que forma $\circ N\left(G_{u}^{\circ *}\right)$

$\xi, \eta=$ coordenadas adimensionais para o elemento finito bidimensional

$\varepsilon_{\ell}=$ medida de deformação linear

$\varepsilon_{\mathrm{g}}=$ medida de deformação de Green 
$\dot{\varepsilon}=$ tensor taxa de deformação de Green

$\dot{\varepsilon}^{*}=$ tensor taxa de deformação de Green na configuração atual

$\varepsilon_{\mathrm{a}}^{*}=$ tensor de deformação de Almansi

$\varepsilon=$ tensor de deformação de Green

$\varepsilon^{*}=$ tensor de deformação de Green na configuração atual

$\varepsilon_{\mathrm{x}}=$ deformação longitudinal em $\mathrm{x}$

$\varepsilon_{y}=$ deformação longitudinal na direção y

$\delta \mathrm{u}_{\mathrm{e}}=$ deslocamento virtual para o elemento finito bidimensional

$\delta U=$ energia de deformação virtual

$\nabla \mathrm{u}_{\mathrm{e}}=$ gradiente do deslocamento para $\mathrm{o}$ elemento finito bidimensional

$\Delta \mathrm{t}=$ incremento de tempo

$\Delta \varphi=$ incremento do autovetor

$\Delta q=$ incremento do vetor deslocamento nodal

$\Delta \lambda=$ incremento do parâmetro de carga

$\delta \dot{\varepsilon}^{*}=$ tensor taxa de deformação virtual de Green na configuração atual

$\delta \dot{\varepsilon}=$ tensor taxa de deformação virtual de Green

$\delta \varepsilon_{\mathrm{a}}^{*}=$ tensor de deformação virtual de Almansi 
$\delta \varepsilon^{*}=$ tensor de deformação virtual de Green na configuração atual

$\delta \varepsilon=$ tensor de deformação virtual de Green na configuração inicial

$\delta \mathrm{W}_{\mathrm{e}}=$ trabalho virtual externo

$\delta \mathrm{W}=$ trabalho virtual total

$\delta q=$ vetor dos deslocamentos virtuais nodais

$\nabla u=$ gradiente dos deslocamentos na configuração inicial

$\gamma_{x y}=$ deformação transversal

$\mathrm{G}_{\alpha}=$ derivada de Frechet na direção de $\alpha$

$\ell(\varphi)=$ função normalizadora do autovetor $\varphi$

$|\hat{\sigma}|=$ matriz representativa do tensor de tensão de Cauchy

$|\hat{S}|=$ matriz representativa do tensor de tensão de Piola-Kirchhoff de $2^{\mathrm{a}}$ espécie

$\lambda=$ parâmetro de carga

$\langle x, y\rangle=$ produto de dualidade entre os vetores $x$ e $y$

$\dot{\mathrm{u}}_{e}=$ velocidade para o elemento finito bidimensional

$\sigma=$ tensor de tensão de Cauchy

$\tau=$ tensor de tensão de Kirchhoff-Treftz 
$S^{\star}=$ tensor de tensão de Piola-Kirchhoff de $2^{\underline{a}}$ espécie na configuração atual

$\dot{S}=$ tensor taxa de tensão de Piola-Kirchhoff de $2^{\mathrm{a}}$ espécie na configuração inicial

$\dot{S}^{*}=$ tensor taxa de tensão de Piola-Kirchhoff de $2^{\underline{a}}$ espécie na configuração atual

$\dot{q}=$ vetor das velocidades nodais

$\tau_{\mathrm{xy}}=$ tensão tangencial no plano $\mathrm{xy}$

$\sigma_{x}=$ tensão normal na direção $x$

$\sigma_{y}=$ tensão normal na direção y

$\hat{n}=$ versor normal à superfície deformada

$\Omega_{\mathrm{e}}=$ volume do elemento finito bidimensional

$\mathrm{X}$ e $\mathrm{Y}$ espaços de Banach

$\mathrm{X}^{*}$ e $\mathrm{Y}^{*}=$ espaços de Banach duais a $\mathrm{X}$ e $\mathrm{Y}$ 


\title{
ABREVIATURAS
}

\author{
PTV $=$ Princípio dos Trabalhos Virtuais \\ P-K = Piola-Kirchhoff \\ $N L G=$ não-linear geométrico, não-linearidade geométrica \\ TOL = tolerância
}




\section{RESUMO}

PAULA, C.F. (1997). Estudo das descrições Lagrangiana e Euleriana na análise não-linear geométrica com o emprego do Método dos Elementos Finitos. São Carlos. 116p. Dissertação (Mestrado). Escola de Engenharia de São Carlos, Universidade de São Paulo.

Neste trabalho estudam-se diversos aspectos conceituais relativos à modelagem mecânico-matemática para a descrição do comportamento estrutural não-linear geométrico. Apresenta-se, inicialmente, a aplicação do Princípio dos Trabalhos Virtuais na caracterização do equilíbrio na posição deslocada. Em seguida, a partir do emprego do Método dos Elementos Finitos, analisam-se as formas discretizadas Lagrangiana e Euleriana da expressão do equilíbrio, que resultam em função da descrição adotada para o movimento. A questão da estabilidade estrutural é tratada, abordando-se os conceitos de ponto limite e de bifurcação. Estudam-se, finalmente, os procedimentos incrementais, em combinação com o Método de Newton, para a solução do sistema não-linear de equações e para a caracterização de pontos singulares de equilíbrio. Exemplos consistindo na análise do comportamento de estruturas reticulares compõem as aplicações numéricas.

Palavras-chave: Método dos Elemento Finitos, não-linearidade geométrica, Princípio dos Trabalhos Virtuais, estabilidade do equilíbrio. 


\section{ABSTRACT}

PAULA, C.F. (1997). Study of the lagrangian and eulerian descriptions in geometrical nonlinear analysis with use of the finite element method. São Carlos. 116p. Dissertação (Mestrado). Escola de Engenharia de São Carlos, Universidade de São Paulo.

In this work, several conceptual aspects related to the mechanicmathematical modeling for description of the geometrical nonlinear stuctural behavior are studied. First of all the Principle of Virtual Work is presented in order to characterize the equilibrium in the displaced position. Then, from the use of finite element method one analyses the lagrangian and eulerian forms which result from the adopted description of the motion. Stability of the structural response is treated by discussing the concepts of limit and bifurcation points. Finally, the incremental procedures in combination with the Newton schemes for solution of nonlinear equations and characterization of the singular points of equilibrium are studied. The numerical applications are related to the analysis of simple linear structures.

Keywords: finite element method, geometrical nonlinearity, Principle of Virtual Work, stability of equilibrium. 


\section{CAPÍTULO 1- INTRODUÇÃO}

$\mathrm{Na}$ mecânica das Estruturas a não-linearidade é usualmente classificada em física, geométrica e decorrente de condições de vinculação.

A característica fundamental da não-linearidade geométrica, tema deste trabalho, é que a condição de equilíbrio é imposta na posição deslocada utilizando-se diretamente o Princípio dos Trabalhos Virtuais ou então formulando-se o problema de análise estrutural através do Método da Energia.

Neste texto o estudo da não-linearidade geométrica é apresentado através da aplicação do PTV, considerando-se exclusivamente a linearidade física. Deste modo, pretende-se oferecer pequenas contribuições para melhor entendimento da modelagem, discutindo-se alguns conceitos básicos relativos tanto a aspectos teóricos quanto de resolução numérica.

Os aspectos teóricos relacionam-se, particularmente, à aplicação de conceitos relativos à mecânica dos meios contínuos, cujo entendimento é essencial para o correto equacionamento do modelo mecânico-matemático do comportamento estrutural. Destacam-se neste caso as diferentes formas de descrição do movimento, a Lagrangiana e a Euleriana, bem como o conceito de medidas conjugadas e objetivas de tensão e deformação, relacionado a estas descrições.

Os aspectos de natureza numérica derivam do fato de que as equações de equilíbrio discretizadas não são lineares. Entre os processos 
de resolução possiveis são discutidos no texto aqueles do tipo incremental ou iterativo, com destaque para o método de Newton-Raphson. Um conceito importante que surge quando se utiliza tal método é o de matriz de rigidez tangente, ilustrado neste trabalho para elementos finitos de barras e de estados planos.

$\mathrm{Na}$ simulação da resposta não-linear geométrica em problemas estruturais é importante, também, realizar uma análise da estabilidade do equilíbrio, procurando-se caracterizar pontos singulares, isto é pontos de bifurcação e limite. Com o objetivo de distingui-los numericamente é necessário inserir as condições de caracterização no algoritmo de resolução numérica. Uma maneira eficiente de fazê-lo é através dos chamados sistemas estendidos, tratados neste trabalho.

Em resumo o presente trabalho tem como objetivo reunir em texto, elementos da formulação via PTV do problema da análise estrutural nãolinear geométrica, expresso em forma geral segundo as descrições Lagrangiana e Euleriana do movimento, e de sua discretização pela aplicação do Método dos Elementos Finitos. Além disso, pretende-se inserir comentários sobre algoritmos numéricos para a caracterização de pontos limites e de bifurcação do equilíbrio. Os principais aspectos conceituais envolvidos são ilustrados em exemplos de aplicação gerados pela utilização de "software" específico já existente.

Para alcançar o objetivo proposto, o texto se encontra organizado como segue.

No capítulo 2, são apresentados os conceitos da mecânica do contínuo necessários ao estudo da não-linearidade geométrica. Tais conceitos estão ligados ao estudo das descrições material e espacial do movimento, definição de deformação e do tensor gradiente de deformação, as medidas de deformação, os tensores de tensão, os tensores de tensão e 
deformação conjugados e a aplicação do Princípio dos Trabalhos Virtuais na formulação do equilíbrio.

No capítulo 3, trata-se da aplicação do Método dos Elementos Finitos à análise não-linear geométrica. Apresentam-se: a forma discretizada das equações de equilíbrio em sua forma incremental considerando-se pequenas deformações, o conceito de matriz de rigidez tangente, as formulações Lagrangiana e Euleriana e as correspondentes matrizes de rigidez para elementos finitos de barras e em estado plano de tensão.

O capítulo 4 é relacionado com a estabilidade do equilíbrio. Apresenta-se a caracterização matemática de pontos limite e de bifurcação e sua introdução nos algoritmos para resolução numérica.

No capítulo 5, são apresentados exemplos numéricos que objetivam ilustrar a aplicação dos conceitos abordados anteriormente na análise de estruturas em regime não-linear geométrico. É oportuno mencionar que não foi desenvolvido um código de cálculo específico. Deste modo, os resultados numéricos dos exemplos de aplicação são gerados com o "software" LUSAS, visto que este permite a análise não-linear de estruturas sob condições gerais de carregamento, dispondo de diferentes algoritmos, entre os quais os do tipo comprimento de arco.

O capítulo 6 reúne as conclusões gerais e sugestões para posteriores pesquisas.

No anexo é apresentado um breve estudo sobre os elementos de análise funcional necessários para a definição de critérios para a caracterização de pontos singulares. 


\section{CAPÍTULO 2- ASPECTOS DA MECÂNICA DO CONTÍNUO}

\section{1- DESCRIÇÃO MATERIAL E ESPACIAL DO MOVIMENTO}

A descrição da deformação de um sólido requer o conhecimento para cada uma de suas partículas, com relação a um certo referencial, das posições onde ela ESTÁ e onde ESTAVA em sucessivos instantes de tempo. Neste trabalho, a coordenada cartesiana $x$ indicará a posição onde a partícula ESTÁ e X onde ela ESTAVA antes da deformação, ver figura 2.1.

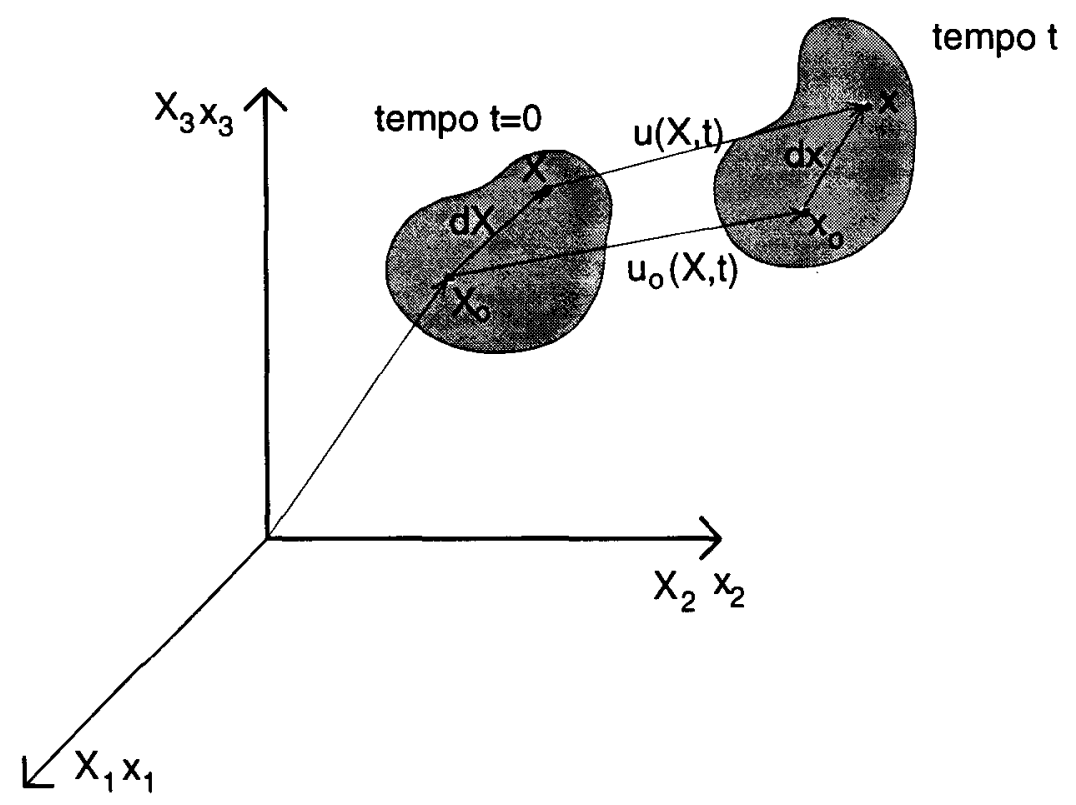

FIGURA 2.1- Partícula em movimento 
Existem duas formas usuais utilizadas para descrever um movimento: são a descrição material e espacial. A descrição espacial ocupa-se do que ocorre em regiões fixas do espaço no decorrer do tempo, enquanto que a descrição material acompanha o movimento do corpo que, com o passar do tempo, ocupa diversas regiões do espaço.

Em uma descrição Material ou Lagrangiana, todo o comportamento é descrito em termos das coordenadas iniciais da partícula $X$, no tempo $t=0$. Assim, a posição de onde a partícula ESTÁ é dada em termos de onde ela ESTAVA por:

$$
x=x(X, t)
$$

Numa descrição Espacial ou Euleriana todo o comportamento é expresso em termos da posição no espaço, correntemente ocupado pela partícula no instante t. Neste caso, a posição onde ela ESTAVA é determinada em termos da posição onde ela ESTÁ por:

$$
X=X(x, t)
$$

Fazendo-se uso da função deslocamento $u$, a qual pode ser expressa em termos de $X$ ou $X$, as relações (2.1) e (2.2) passam a ser dadas por:

$$
\begin{aligned}
& x=X+u(X, t) \\
& X=x-u(x, t)
\end{aligned}
$$

\section{2- TENSORES DE DEFORMAÇÃO}

Para duas partículas vizinhas $X_{0}$ e $X$, mostradas na figura 2.1, no tempo $t=0$ a posição relativa inicial é $d X$. Em função da deformação, no tempo $t$ a posição relativa passa $a \mathrm{dx}$. Usando-se a equação (2.1), a relação entre $d x$ e $d X$ é dada por: 


$$
d x=F d X \quad \text { e } \quad F=\frac{\partial x}{\partial X}
$$

Onde $F$ é denominado tensor gradiente de deformação e é a quantidade fundamental que descreve a deformação de um sólido, pois permite estabelecer a relação de uma 'fibra' material $d X$ antes da deformação com a 'fibra' dx depois da deformação.

Quando as posições inicial e atual da partícula são relacionadas pela função deslocamento dada por $x=X+u(X, t)$, 0 gradiente de deformação passa ser:

$$
F=\left[I+\frac{\partial u}{\partial X}\right]
$$

onde l é o tensor identidade e $\frac{\partial u}{\partial X}(o u \nabla u)$ é chamado de tensor gradiente do deslocamento. É possivel mostrar que a parte simétrica desse tensor se relaciona com a medida linear de deformação. No entanto, outros tensores de deformação são de interesse e se relacionam com as medidas quadráticas de deformação.

Assim, utilizando-se a equação (2.4), pode-se definir uma medida quadrática da variação de comprimento por:

$$
\|d X\|^{2}-\|d X\|^{2}=F^{\top} F d X \cdot d X-d X \cdot d X=\left(F^{\top} F-1\right) d X \cdot d X=2 \varepsilon d X . d X
$$

Onde:

$$
\begin{aligned}
& \varepsilon=\frac{1}{2}\left[F^{\top} F-l\right]=\frac{1}{2}\left[\left(\frac{\partial u}{\partial X}\right)+\left(\frac{\partial u}{\partial X}\right)^{\top}+\left(\frac{\partial u}{\partial X}\right)^{\top}\left(\frac{\partial u}{\partial X}\right)\right] \text { ou } \\
& \varepsilon=\frac{1}{2}\left[\nabla u+\nabla u^{\top}+\nabla u^{\top} \nabla u\right]
\end{aligned}
$$

$\varepsilon$ é denominado tensor das deformações de Green. 
No entanto, a equação (2.4) pode ser escrita em termos do comprimento inicial $\mathrm{dX}$, tal que:

$$
d X=F^{-1} d x
$$

Assim, utilizando-se a equação (2.7), pode-se definir uma medida quadrática da variação de comprimento, agora em função do comprimento final $d x$ :

$$
\|d x\|^{2}-\|d X\|^{2}=d x \cdot d x-F^{-T} F^{-1} d x \cdot d x=\left(I-F^{-T} F^{-1}\right) d x \cdot d x=2 \varepsilon_{a}^{*} d x \cdot d x
$$

Onde:

$$
\varepsilon_{\mathrm{a}}^{*}=\frac{1}{2}\left[1-\left(\mathrm{F}^{-1}\right)^{\top}\left(\mathrm{F}^{-1}\right)\right]=\frac{1}{2}\left[\left(\frac{\partial \mathrm{u}}{\partial \mathrm{x}}\right)+\left(\frac{\partial \mathrm{u}}{\partial \mathrm{x}}\right)^{\top}-\left(\frac{\partial \mathrm{u}}{\partial \mathrm{x}}\right)^{\top}\left(\frac{\partial \mathrm{u}}{\partial \mathrm{x}}\right)\right]
$$

$\varepsilon_{\mathrm{a}}^{*}$ é o tensor de deformação de Almansi.

Observa-se que no tensor de Green os gradientes são tomados com relação à posição inicial e no de Almansi com relação à posição atual da partícula.

\section{3- TENSORES DE TENSÃO}

Sabe-se que na análise não-linear geométrica as equações de equilíbrio devem se referir à configuração deformada da estrutura no tempo $t$, mas como a geometria na configuração deformada é desconhecida, as equações podem ser convenientemente escritas em termos de medidas tomadas na configuração indeformada. No processo de transformação dessas equações surgem diferentes definições de tensores de tensão.

Seja o corpo, ou uma parte dele, nas configurações inicial e atual representados pela figura 2.2 . 

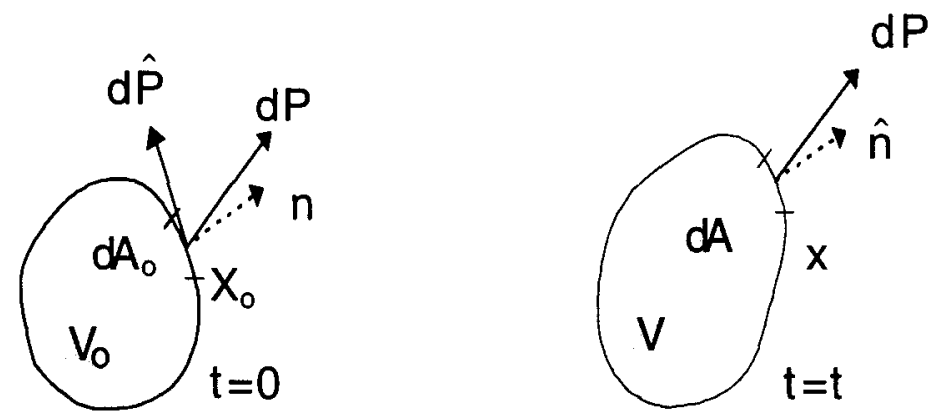

FIGURA 2.2- Corpo sujeito a tensões

O tensor de tensões associado à partícula na configuração deformada, é chamado de tensor de Cauchy (simétrico) e representado por $\sigma . O$ tensor de Cauchy $(\sigma)$ determina o vetor de tensão atual (tempo t) por unidade de área deformada. Consequentemente na figura 2.2 tem-se que:

$$
d P=\sigma \hat{n} d A
$$

onde n̂ é o versor normal à superfície atual.

A simples multiplicação de $\sigma$ pelo determinante de $\mathrm{F}$ define-se 0 tensor de tensão de Kirchhoff-Treftz:

$$
\tau=\mathrm{J} \sigma
$$

onde $\mathrm{J}=\operatorname{det} \mathrm{F}$.

Para exprimir $\mathrm{dP}$ em termos da área indeformada $\left(\mathrm{dA} \mathrm{A}_{\circ}\right)$, define-se um novo tensor de tensão $\Pi$, chamado tensor de tensão de Piola-Kirchhoff de $1^{\text {a }}$ espécie, dado por:

$$
d P=\Pi n d A_{。}
$$

onde $\mathrm{n}$ é o versor normal à supertície indeformada. 
A relação entre o tensor de Cauchy e o de Piola-Kirchhoff de $1^{\text {a }}$ espécie pode ser explicitada fazendo-se uso da relação de Nanson $\hat{n} d A=(\operatorname{det} F) F^{-T} n d A_{0}$. Deste modo, resulta:

$$
\Pi=(\operatorname{det} F) \sigma F^{-T}
$$

Da relação (2.10) observa-se que para uma dada normal unitária $n$, na configuração indeformada, o tensor de P-K $1^{\underline{a}}$ espécie dá a força resultante atual com referência à área indeformada. Esse tensor é nãosimétrico.

Existe outro tensor de tensão associado aos nomes de Piola e Kirchhoff chamado tensor de tensão de Piola-Kirchhoff de $2^{a}$ espécie e é representado por $S$. A esse tensor (simétrico) pode ser associada uma interpretação como se mostra a seguir. Do mesmo modo que $d X=F^{-1} d x$, admite-se que dP possa se transformar da configuração atual para a inicial tal como $d \hat{P}=F^{-1} d P$. Então tem-se:

$$
d \hat{P}=\operatorname{Snd} A_{\circ}
$$

Empregando-se novamente a relação de Nanson conclui-se que:

$$
S=F^{-1} \Pi
$$

Consequentemente, para uma dada normal unitária $n$, na configuração indeformada, a tensão de $\mathrm{P}-\mathrm{K} 2^{\underline{a}}$ espécie dá com referência à configuração inicial a força atual transformada pelo inverso do mesmo tensor gradiente de deformação, que faz passar da configuração inicial para a atual.

As diferentes definições para os tensores de tensão relacionam-se através do tensor $F$ : 


$$
\begin{aligned}
& \Pi^{\top}=\mathrm{JF}^{-1} \sigma=\mathrm{SF}^{\top} \\
& \mathrm{S}=\mathrm{J}\left(\mathrm{F}^{-1}\right) \sigma\left(\mathrm{F}^{-1}\right)^{\top}=\Pi^{\top}\left(\mathrm{F}^{-1}\right)^{\top}
\end{aligned}
$$

Em particular, lembrando-se que $F$ pode ser expresso pela composição de um tensor de rotação $R$ e outro de deformação $U$ como $F=R U$, para pequenas deformações pode-se admitir que $U \cong I \rightarrow F \cong R$, $\operatorname{assim} S=R^{\top} \sigma R$.

Observa-se que $\sigma, \tau$ e $S$ são simétricos enquanto $\Pi$ é não simétrico.

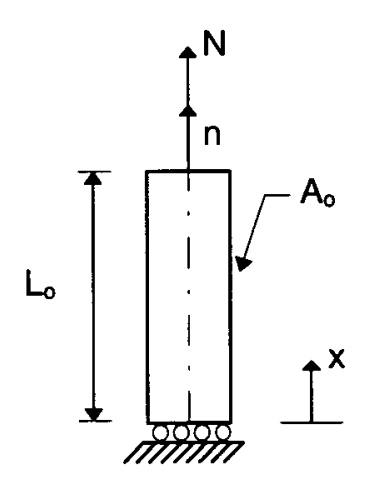

(a)

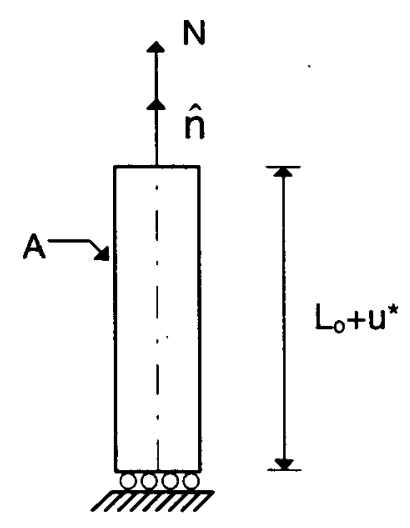

(b)

FIGURA 2.3- Barra axialmente tracionada

Como exemplo de ilustração, de acordo com a figura 2.3 tem-se que a tensão de Cauchy pode ser escrita por:

$$
\sigma=\frac{N}{A}
$$

No caso da barra axialmente tracionada, a função deslocamento u pode ser descrita por: 


$$
u=\frac{u^{*}}{L_{o}} X
$$

Deste modo o gradiente de deformação é dado por:

$$
F=1+\frac{u^{*}}{L_{0}}=F^{\top} \quad F^{-1}=\frac{L_{0}}{L_{o}+u^{*}}
$$

Utilizando-se a relação entre volumes inicial e deformado, MALVERN (1969), $\operatorname{det} F=\frac{V}{V_{0}}=\frac{\left(L_{0}+u^{*}\right)}{L_{o}} \frac{A}{A_{0}}$ na equação (2.11), tem-se que a tensão de Piola-Kirchhoff de $1^{\text {a }}$ espécie é dada por:

$$
\begin{aligned}
& \Pi=\frac{A}{A_{o}} \frac{\left(L_{o}+U^{*}\right)}{L_{o}} \sigma \frac{L_{o}}{\left(L_{o}+U^{*}\right)}=\frac{A}{A_{o}} \sigma \\
& \text { ou } \Pi=\frac{N}{A_{o}} \text { que coincide com a chamada tensão nominal. }
\end{aligned}
$$

\section{4- PRINCIPIO DOS TRABALHOS VIRTUAIS}

Em forma local, isto é, em cada ponto do corpo deformado, o equilíbrio é expresso por seis equações independentes, representando o equilíbrio à translação e o equilíbrio à rotação segundo três direções independentes. Pelo PTV, através de uma única equação escalar simples, é possivel exprimir o equilíbrio para todo o corpo. 


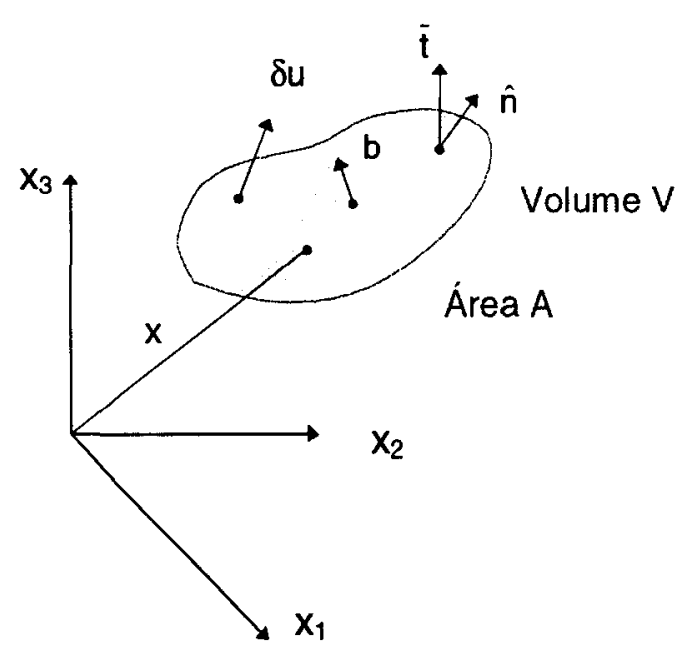

FIGURA 2.4- Princípio dos trabalhos virtuais para um sólido - HINTON (1993)

b e $\bar{t}$ são as forças por unidade de volume e por unidade de área respectivamente.

Para o 'corpo' mostrado na figura 2.4, a forma local da equação diferencial Euleriana de equilíbrio é:

$$
r=(\operatorname{div} \sigma)+b=0
$$

Por outro lado, definindo-se um campo de deslocamentos virtuais infinitesimais como:

$$
\delta u=\delta u(X)=\delta u(x, t)
$$

se $r=0$, então uma equação de trabalho virtual pode ser construída como $\delta \mathrm{W}=\int_{V} r . \delta \mathrm{d} \mathrm{V}=0$. Usando-se $\quad 0 \quad$ teorema da divergência $\left(\int_{A} \sigma \hat{n} d A=\int_{V} \operatorname{div} \sigma d V\right)$ junto com $\bar{t}=\sigma \hat{n}$, esta equação de trabalho virtual pode ser escrita na forma:

$$
\delta W=\delta U-\delta W_{e x}=0
$$

onde a energia de deformação virtual é dada por: 


$$
\delta \mathrm{U}=\int_{\mathrm{V}} \sigma \cdot \delta \varepsilon_{\mathrm{a}}^{*} \mathrm{dV}
$$

e o trabalho virtual externo é:

$$
\delta W_{e x}=\int_{V} b \cdot \delta u d V+\int_{A} \bar{t} . \delta u d A
$$

Em poucas palavras o Princípio dos Trabalhos Virtuais afirma que:

"Se $\delta U-\delta W_{e x}=0$ para todo $\circ$ campo de deslocamentos virtuais cinematicamente admissíveis (isto é, satisfazendo às condições de contorno e compatibilidade) então, o estado de tensão satisfaz as condições de equilíbrio no interior e no contorno."

Assim, a expressão $\int_{V} \sigma . \delta \varepsilon_{a}^{*} d V-\int_{V}^{b}$. $\delta u d V-\int_{A}^{\bar{t}} \cdot \delta u d A=0$ fornece a forma espacial ou Euleriana do equilíbrio.

Infelizmente, o PTV expresso em termos de integrais sobre um volume $\mathrm{V}$ e área superficial $\mathrm{A}$ atuais, desconhecidos, não é prático e uma transformação é necessária de modo que essas integrais sejam referidas ao volume $V_{o}$ e à área $A_{o}$ iniciais conhecidos. Com o objetivo de realizar essa transformação é importante lembrar que um elemento de volume $d V_{o}$ antes da deformação, está relacionado ao volume $d V$ depois da deformação por $d V=J d V_{0}$, onde $J=\operatorname{det} F$ e que, por sua vez, a relação entre $d A_{0}$ e $d A$ elementos de área antes e depois da deformação, é dada, através da relação de Nanson, por $n d A_{o}=J^{-1} F^{\top} \hat{n} d A$. Resultam portanto, as formas Material ou Lagrangiana do PTV, onde as duas integrais de interesse são:

$$
\begin{aligned}
& \int_{V_{0}} r_{0} \cdot \delta u d V_{o}=\int_{V_{0}} T \cdot \nabla \delta u d V_{o}-\int_{V_{0}} b_{0} \cdot \delta u d V_{o}-\int_{A_{0}} \bar{t}_{0} \cdot \delta u d A_{o}=0 \\
& \int_{V_{0}} r_{0} \cdot \delta u d V_{0}=\int_{V_{0}} S \cdot \delta \varepsilon d V_{0}-\int_{V_{0}} b_{0} \cdot \delta u d V_{o}-\int_{A_{0}} \bar{t}_{0} \cdot \delta u d A_{o}=0
\end{aligned}
$$


tal que $\nabla \delta u=\frac{\partial \delta u}{\partial X} ; r_{0}=J r ; b_{o}=J b$ e $\bar{t}_{o}=\left(\frac{d A}{d A_{0}}\right) \bar{t}$.

Nas equações (2.17), (2.18) e (2.19) deve-se considerar que a energia virtual de deformação $\delta U$ é a mesma independente do volume sobre - qual a integral está sendo tomada. Consequentemente, a diferentes medidas de deformação virtual devem corresponder diferentes tensões de modo a manter $\delta U$ invariante, aparecendo portanto o conceito de tensão e deformação conjugadas, MALVERN (1969). Por exemplo, medidas de tensão e deformação conjugadas são: o tensor de $\mathrm{P}-\mathrm{K}$ de $2^{\mathrm{a}}$ espécie conjugado com o tensor de deformação virtual de Green $(S, \delta \varepsilon)$, o tensor de $\mathrm{P}-\mathrm{K}$ de $1^{\underline{a}}$ espécie com 0 tensor gradiente dos deslocamentos verticais $\left(\Pi, \frac{\partial \delta \mathrm{u}}{\partial \mathrm{X}}\right)$ e o tensor de Cauchy com o de deformação virtual de Almansi $\left(\sigma, \delta \varepsilon_{a}^{*}\right)$.

Observa-se, ainda, que na equação (2.19) o tensor de deformação virtual de Green é dado por:

$$
\delta \varepsilon=\frac{1}{2}\left[\nabla \delta \mathrm{u}+(\nabla \delta \mathrm{u})^{\top}+(\nabla \delta \mathrm{u})^{\top} \nabla \mathrm{u}+(\nabla \mathrm{u})^{\top} \nabla \delta \mathrm{u}\right]
$$




\section{CAPÍTULO 3- APLICAÇÃO DO MÉTODO DOS ELEMENTOS FINITOS}

\section{1- EQUAÇÕES DE EQUILÍBRIO DISCRETIZADAS}

Como visto anteriormente, as expressões do PTV essencialmente não são lineares nos deslocamentos, sugerindo-se portanto sua linearização combinada com um procedimento iterativo, para encontrar a solução.

Inicialmente é importante observar que as análises em questão neste trabalho desconsideram efeitos dinâmicos, de modo que a variável tempo é interpretada como um parâmetro implícito controlador da deformação da estrutura. Seja então a equação de equilíbrio (2.19), relativa à formulação Lagrangiana Total e admita-se que um campo de deslocamentos tenha sido encontrado satisfazendo esta equação no instante t. O problema de análise estrutural consiste em encontrar o campo de deslocamento no tempo $t+\Delta t$, sabendo-se que a equação do PTV não é linear. O método de Newton:-Raphson surge da linearização da equação de equilíbrio, realizada a partir do truncamento, em $1^{\underline{a}}$ ordem no tempo, da equação (2.19) desenvolvida em série em torno do tempo $t$, tal como:

$$
\left(\int_{V_{0}} r_{0} \cdot \delta u d V_{0}\right)_{t+\Delta t}=\left(\int_{V_{0}} r_{0} \cdot \delta u d V_{0}\right)_{t}+\frac{d}{d t}\left(\int_{V_{0}} r_{0} \cdot \delta u d V_{0}\right)_{t} \Delta t=0
$$


Restringindo-se ao caso de forças que não são dependentes da deformação (forças conservativas) torna-se possivel escrever a derivada que aparece na relação (3.1) como:

$$
\begin{aligned}
\frac{d}{d t} \int_{V_{0}} r_{0} \cdot \delta u d V_{0} & =\int_{V_{0}} \dot{S} \cdot \delta \varepsilon d V_{0}+\int_{V_{0}} S \cdot\left[\left(\frac{\partial \delta u}{\partial X}\right)^{\top}\left(\frac{\partial \dot{u}}{\partial X}\right)\right] d V_{0}+ \\
& -\int_{V_{0}} \dot{b}_{0} \cdot \delta u d V_{0}-\int_{A_{0}} \dot{\bar{t}}_{0} \cdot \delta u d A_{0}
\end{aligned}
$$

Porém, se a descrição for a Lagrangiana Atualizada, em que a configuração é a atualizada no tempo t, a equação (3.2) passa a ser escrita como:

$$
\begin{aligned}
\frac{d}{d t} \int_{V} r . \delta u d V= & \int_{V} \dot{S}^{*} . \delta \varepsilon^{*} d V+\int_{V} \sigma \cdot\left[\left(\frac{\partial \delta u}{\partial x}\right)^{\top}\left(\frac{\partial \dot{u}}{\partial x}\right)\right] d V+ \\
& -\int_{V} \dot{b} . \delta u d V-\int_{A} \dot{\dot{t}} . \delta u d A
\end{aligned}
$$

onde ù é a velocidade.

Para a equação (3.2), tem-se que o tensor taxa de deformação de Green, ou tensor velocidade de deformação, é dado por:

$$
\dot{\varepsilon}=\frac{1}{2}\left[\left(\frac{\partial \dot{u}}{\partial X}\right)+\left(\frac{\partial \dot{u}}{\partial X}\right)^{\top}+\left(\frac{\partial \dot{u}}{\partial X}\right)^{\top}\left(\frac{\partial u}{\partial X}\right)+\left(\frac{\partial u}{\partial X}\right)^{\top}\left(\frac{\partial \dot{u}}{\partial X}\right)\right]
$$

e o tensor taxa de deformação virtual:

$$
\delta \dot{\varepsilon}=\left(\frac{\partial \delta u}{\partial X}\right)^{\top}\left(\frac{\partial \dot{u}}{\partial X}\right)
$$


Para a descrição Lagrangiana Atualizada, na equação (3.3), as equações (3.4a) e (3.4b) devem ser escritas com relação à última configuração equilibrada.

\section{2- EQUAÇÕES INCREMENTAIS DE EQUILÍBRIO}

A equação incremental de equilíbrio com referência a um certo instante de tempo $t+\Delta t$ é:

$$
\left(\int_{V_{0}} r_{0} \cdot \delta u d V_{o}\right)_{t}+\frac{d}{d t}\left(\int_{V_{0}} r_{0} \cdot \delta u d V_{o}\right)_{t} \Delta t=0
$$

que após algumas operações, que serão detalhadas mais adiante, pode ser reescrita na forma:

$$
(r)_{t}+\left(K_{T} \dot{u}-\dot{f}_{b}-\dot{f}_{\bar{t}}\right)_{t} \Delta t=0
$$

onde $o$ vetor de forças residuais $(r)_{t}$ é determinado pela diferença entre a resultante das tensões internas e as cargas aplicadas, $\mathrm{K}_{\mathrm{T}}$ é a matriz de rigidez tangente, ú é o vetor de velocidades nodais, $\dot{f}_{b}$ e $\dot{f}_{\dot{f}}$ são as taxas das forças por unidade de volume e de superfície respectivamente e $\Delta t$ é um intervalo de tempo onde ocorre o incremento dos deslocamentos $\Delta \mathrm{u}$.

Pode-se ainda escrever a equação incremental de equilíbrio como:

$$
(r)_{t}+K_{T} \Delta u-\Delta f_{b}-\Delta f_{\bar{t}}=0
$$

onde: $\Delta \mathrm{u}=\dot{\mathrm{u}} \Delta \mathrm{t}, \Delta \mathrm{f}_{\mathrm{b}}=\dot{\mathrm{f}}_{\mathrm{b}} \Delta \mathrm{t}$ e $\Delta \mathrm{f}_{\mathrm{t}}=\dot{\mathrm{f}}_{\mathrm{t}} \Delta \mathrm{t}$

A discretização da estrutura pela técnica dos elementos finitos leva a uma forma aproximada para as equações incrementais de equilíbrio. 
Com esse objetivo, é importante reescrever em forma matricial as equações de equilíbrio e da taxa de equilíbrio. Assim resultam:

(a) para a relação de equilibrio

$$
\int_{V_{0}} r_{0}^{\top} \delta u d V_{o}=\int_{V_{0}} \delta \varepsilon^{\top}\{S\} d V_{0}-\int_{V_{0}} b_{0}^{\top} \delta u d V_{0}-\int_{A_{0}} \bar{t}_{0}^{\top} \delta u d A_{0}=0
$$

(b) para a taxa de equilíbrio

$$
\frac{d}{d t} \int_{V_{0}} r_{0}^{\top} \delta u d V_{0}=\frac{d}{d t} \int_{V_{0}} \delta \varepsilon^{\top}\{S\} d V_{o}-\int_{V_{0}} \dot{b}_{0}^{\top} \delta u d V_{o}-\int_{A_{0}} \dot{\vec{t}}_{0}^{\top} \delta u d A_{0}
$$

\section{3- MATRIZ DE RIGIDEZ TANGENTE}

Como visto na equação (3.6), a matriz de rigidez tangente resultou de uma análise incremental, ou seja da linearização da equação de equilíbrio. Deste modo, pode-se dizer que a matriz de rigidez tangente nas análises incrementais surge quando se usa o processo de Newton-Raphson.

Logo, da equação (3.6) tem-se que a expressão para a matriz de rigidez tangente considerando-se forças conservativas, surge de:

$$
\frac{d}{d t} \int_{V_{0}} S \cdot \delta \varepsilon d V_{0} \Rightarrow K_{T}
$$

$\mathrm{Na}$ equação (3.7), observa-se que a matriz de rigidez tangente relaciona os acréscimos de deslocamentos com as variações dos esforços aplicados.

Pode-se adiantar que a matriz de rigidez tangente é composta por três parcelas: a matriz de rigidez elástica linear, a matriz das correções devidas à mudança das coordenadas nodais e a matriz de rigidez geométrica, que leva em consideração o esforço axial, como será mostrado mais adiante. $\mathrm{Na}$ maioria dos casos, a matriz de rigidez tangente é 
indispensável para a verificação da existência de pontos singulares na análise da estabilidade do equilibrio, como será discutido no capítulo 4 .

\section{4- FORMULAÇÕES LAGRANGIANA TOTAL E ATUALIZADA EM ELEMENTOS FINITOS}

A maioria dos programas em elementos finitos que permitem considerar a não-linearidade geométrica são baseados em alguma forma de descrição Lagrangiana do equilíbrio. Numa formulação Lagrangiana Total todas as integrais são calculadas com relação à configuração inicial indeformada da estrutura no tempo $t=0$. Alternativamente, uma configuração deformada conhecida pode ser tomada como um estado inicial e continuamente atualizada ao longo da análise, esta é chamada formulação Lagrangiana Atualizada.

Nesta seção, utilizando-se o Princípio dos Trabalhos Virtuais em combinação com o Método dos Elementos Finitos, obtêm-se as formas discretizadas da matriz de rigidez tangente correspondentes às formulações Lagrangiana Total e Atualizada, para aplicação em análises de estruturas de barras e estados planos.

\subsection{1- FORMULAÇÃO LAGRANGIANA TOTAL}

\subsubsection{1- ELEMENTO FINITO DE TRELIÇA PLANA}

Considere-se o elemento de barra de treliça num sistema local, mostrado na figura 3.1, com quatro graus de liberdade, sendo dois por nó, correspondentes aos deslocamentos na direção do eixo da barra e transversal a ele. $O$ elemento possui, na sua configuração inicial, área de seção transversal $A_{o}$ e comprimento $L_{0}$. $O$ material é considerado elástico linear. 


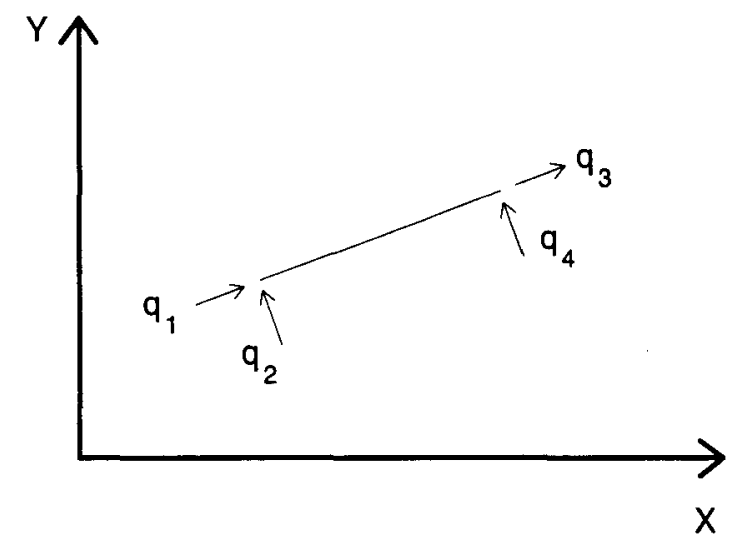

FIGURA 3.1- Elemento finito de treliça plana

Para o elemento finito mostrado na figura 3.1, os deslocamentos são interpolados de maneira usual por:

$$
u=\left\{\begin{array}{l}
u_{1} \\
u_{2}
\end{array}\right\}=M q
$$

Sendo:

$u_{1}$ - componente do deslocamento na direção do eixo da barra

$\mathrm{u}_{2}$ - componente do deslocamento na direção transversal ao eixo da barra

onde:

$$
M=\left[\begin{array}{cccc}
N_{1} & 0 & N_{3} & 0 \\
0 & N_{2} & 0 & N_{4}
\end{array}\right]
$$

e as funções interpoladoras e o vetor dos deslocamentos nodais são:

$$
N_{1}(X)=N_{2}(X)=\left(1-\frac{X}{L_{0}}\right)
$$




$$
\begin{aligned}
& N_{3}(X)=N_{4}(X)=\frac{X}{L_{0}} \\
& q^{\top}=\left\{\begin{array}{llll}
q_{1} & q_{2} & q_{3} & q_{4}
\end{array}\right\}
\end{aligned}
$$

O gradiente dos deslocamentos pode então ser expresso por:

$$
\nabla u=\left[\begin{array}{l}
\frac{\partial u_{1}}{\partial X} \\
\frac{\partial u_{2}}{\partial X}
\end{array}\right] \Rightarrow \begin{aligned}
& \frac{\partial u_{1}}{\partial X}=-\frac{1}{L_{0}} q_{1}+\frac{1}{L_{0}} q_{3} \\
& \frac{\partial u_{2}}{\partial X}=-\frac{1}{L_{0}} q_{2}+\frac{1}{L_{0}} q_{4}
\end{aligned}
$$

ou na forma matricial por:

$$
\nabla u=G q \text { onde } G=\frac{1}{L_{0}}\left[\begin{array}{cccc}
-1 & 0 & 1 & 0 \\
0 & -1 & 0 & 1
\end{array}\right]
$$

Por sua vez, a deformação de Green tem uma única componente ativa, $\varepsilon_{11}$, dada por:

$$
\varepsilon_{11}=\left(\frac{\partial u_{1}}{\partial X}\right)+\frac{1}{2}\left[\left(\frac{\partial u_{1}}{\partial X}\right)^{2}+\left(\frac{\partial u_{2}}{\partial X}\right)^{2}\right]
$$

Os deslocamentos virtuais são interpolados de modo análogo como $\delta u=M \delta q$. Usando-se a equação (2.20), a deformação virtual de Green resulta:

$$
\delta \varepsilon_{11}=\left(\frac{\partial \delta \mathrm{u}_{1}}{\partial \mathrm{X}}\right)+\left(\frac{\partial \delta \mathrm{u}_{1}}{\partial \mathrm{X}}\right)\left(\frac{\partial \mathrm{u}_{1}}{\partial \mathrm{X}}\right)+\left(\frac{\partial \delta \mathrm{u}_{2}}{\partial \mathrm{X}}\right)\left(\frac{\partial \mathrm{u}_{2}}{\partial \mathrm{X}}\right)
$$

Efetuando-se as derivadas da equação acima e escrevendo-se na forma matricial tem-se: 


$$
\begin{aligned}
& \delta \varepsilon_{11}=\left\{\left(\begin{array}{llll}
-\frac{1}{L_{0}} & 0 & \frac{1}{L_{0}} & 0
\end{array}\right)+\right. \\
& \left.+\frac{1}{L_{0}^{2}}\left[\begin{array}{llll}
-\left(q_{3}-q_{1}\right) & -\left(q_{4}-q_{2}\right) & \left(q_{3}-q_{1}\right) & \left(q_{4}-q_{2}\right)
\end{array}\right]\right\}\left\{\begin{array}{l}
\delta q_{1} \\
\delta q_{2} \\
\delta q_{3} \\
\delta q_{4}
\end{array}\right\}
\end{aligned}
$$

Essa última relação pode ser representada por:

$$
\delta \varepsilon_{11}=\mathrm{B} \delta q \text { tal que } \mathrm{B}=\mathrm{B}_{\mathrm{o}}+\mathrm{B}_{\mathrm{L}}
$$

onde:

$$
\begin{aligned}
& B_{o}=\left[\begin{array}{llll}
-\frac{1}{L_{0}} & 0 & \frac{1}{L_{0}} & 0
\end{array}\right] \text { e } \\
& B_{L}=\frac{1}{L_{o}^{2}}\left[\begin{array}{llll}
-\left(q_{3}-q_{1}\right) & -\left(q_{4}-q_{2}\right) & \left(q_{3}-q_{1}\right) & \left(q_{4}-q_{2}\right)
\end{array}\right]
\end{aligned}
$$

A equação constitutiva para problemas envolvendo pequenas deformações e grandes deslocamentos, pode ser escrita por:

$$
\{\dot{\mathbf{s}}\}=\mathrm{D}\{\dot{\varepsilon}\}
$$

onde $\mathrm{D}$ é a matriz constitutiva do material.

O gradiente dos deslocamentos virtuais é dado por:

$$
\nabla \delta \mathrm{u}=\mathrm{G} \delta q
$$

Empregando-se as equações (3.4a) e (3.4b), tem-se que a taxa de deformação de Green para o elemento de barra de treliça plana é, por sua vez, dada por: 


$$
\dot{\varepsilon}_{11}=\left(\frac{\partial \dot{u}_{1}}{\partial X}\right)+\left(\frac{\partial u_{1}}{\partial X}\right)\left(\frac{\partial \dot{u}_{1}}{\partial X}\right)+\left(\frac{\partial u_{2}}{\partial X}\right)\left(\frac{\partial \dot{u}_{2}}{\partial X}\right)=B \dot{q}
$$

e a taxa de deformação virtual de Green por:

$$
\delta \dot{\varepsilon}_{11}^{\top}=\delta q^{\top} G^{\top} G \dot{q}
$$

Utilizando-se a equação (3.9) e desconsiderando-se forças atuantes no elemento, obtém-se:

$$
\delta q^{\top} \dot{r}=\delta q^{\top} K_{T} \dot{q}
$$

onde:

$$
\frac{\mathrm{d}}{\mathrm{dt}} \int_{V_{0}}\{S\}^{\top} \delta \varepsilon d V_{o}=\delta q^{\top} K_{T} \dot{q}
$$

Sabendo-se que:

$$
\frac{\mathrm{d}}{\mathrm{dt}} \int_{V_{0}}\{S\}^{\top} \delta \varepsilon d V_{o}=\int_{V_{0}} \delta \varepsilon^{\top}\{\dot{S}\} d V_{o}+\int_{V_{0}} \delta \dot{\varepsilon}^{\top}|\hat{S}| d V_{o}
$$

e efetuando-se as operações indicadas, a matriz de rigidez tangente para um elemento finito de treliça plana resulta de:

$$
\begin{gathered}
K_{T}=\int_{V_{0}} B^{T} D_{11} B d V_{0}+\int_{V_{0}} G^{\top} S_{11} G d V_{0}= \\
=\int_{V_{0}}\left(B_{0}^{\top} E B_{0}+B_{0}^{T} E B_{L}+B_{L}^{\top} E B_{0}+B_{L}^{\top} E B_{L}\right) d V_{0}+\int_{V_{0}} G^{\top} S_{11} G d V_{0}
\end{gathered}
$$

a qual pode ser também escrita na forma:

$$
\mathrm{K}_{\mathrm{T}}=\mathrm{K}_{\mathrm{o}}+\mathrm{K}_{\mathrm{L}}+\mathrm{K}_{\sigma}
$$

sendo, cada parcela dada por: 


$$
\begin{aligned}
& K_{0}=\int_{V_{0}} B_{0}^{\top} E B_{0} d V_{0} \\
& K_{L}=\int_{V_{0}}\left(B_{0}^{\top} E B_{L}+B_{L}^{\top} E B_{0}+B_{L}^{\top} E B_{L}\right) d V_{0} \\
& K_{\sigma}=\int_{V_{0}} G^{\top} S_{11} G d V_{0}
\end{aligned}
$$

Onde $D_{11}=E, K_{0}$ é a matriz de rigidez elástica linear, $K_{L}$ é matriz de correção das coordenadas e $K_{\sigma}$ é a matriz de rigidez geométrica que é função do nível de solicitação axial nas barras.

Realizando-se as operações indicadas em cada parcela da matriz de rigidez tangente, chega-se às seguintes matrizes:

$$
\begin{aligned}
& K_{\circ}=\frac{E A_{\circ}}{L_{\circ}}\left[\begin{array}{cccc}
1 & 0 & -1 & 0 \\
0 & 0 & 0 & 0 \\
-1 & 0 & 1 & 0 \\
0 & 0 & 0 & 0
\end{array}\right] \quad K_{\sigma}=\frac{N}{L_{0}}\left[\begin{array}{cccc}
1 & 0 & -1 & 0 \\
0 & 1 & 0 & -1 \\
-1 & 0 & 1 & 0 \\
0 & -1 & 0 & 1
\end{array}\right] \\
& K_{L}=\frac{E A_{\circ}}{L_{0}^{2}}\left[\begin{array}{cccc}
2 \hat{U}_{1} & \hat{U}_{2} & -2 \hat{U}_{1} & -\hat{U}_{2} \\
\hat{U}_{2} & 0 & -\hat{U}_{2} & 0 \\
-2 \hat{U}_{1} & -\hat{U}_{2} & 2 \hat{U}_{1} & \hat{U}_{2} \\
-\hat{U}_{2} & 0 & \hat{U}_{2} & 0
\end{array}\right]+ \\
& +\frac{E A_{0}}{L_{0}^{3}}\left[\begin{array}{cccc}
\left(\hat{U}_{1}\right)^{2} & \hat{U}_{1} \hat{U}_{2} & -\left(\hat{U}_{1}\right)^{2} & -\hat{U}_{1} \hat{U}_{2} \\
\hat{U}_{2} & \left(\hat{U}_{2}\right)^{2} & -\hat{U}_{1} \hat{U}_{2} & -\left(\hat{U}_{2}\right)^{2} \\
-\left(\hat{U}_{1}\right)^{2} & -\hat{U}_{1} \hat{U}_{2} & \left(\hat{U}_{1}\right)^{2} & \hat{U}_{1} \hat{U}_{2} \\
-\hat{U}_{1} \hat{U}_{2} & -\left(\hat{U}_{2}\right)^{2} & \hat{U}_{1} \hat{U}_{2} & \left(\hat{U}_{2}\right)^{2}
\end{array}\right]
\end{aligned}
$$

onde $\hat{U}_{1}=\left(q_{3}-q_{1}\right)$ e $\hat{U}_{2}=\left(q_{4}-q_{2}\right)$. 


\subsubsection{2- ELEMENTO FINITO DE PÓRTICO PLANO}

Considere-se o elemento de barra de pórtico plano mostrado na figura 3.2, com seis graus de liberdade, sendo 3 por nó: o deslocamento na direção do eixo, o deslocamento transversal e a rotação em torno do eixo perpendicular ao plano $\mathrm{XY}$ da barra. $\mathrm{O}$ material é considerado elástico linear. $O$ elemento possui na sua configuração inicial área de seção transversal $A_{0}$ e comprimento $L_{0}$. Admite-se a seguinte hipótese sobre 0 campo de deslocamentos: as seções transversais permanecem planas, ortogonais ao eixo deslocado e não alteram sua geometria após deformação (hipóteses de Navier). Assim, conhecendo-se os deslocamentos axiais e transversais dos pontos do eixo da barra, o deslocamento de um ponto genérico pertencente à seção transversal, como mostra a figura 3.3 , é descrito por:

$$
\begin{aligned}
& u(X, Y)=u_{0}(X)-Y v_{0}^{\prime} \\
& v(X, Y)=v_{o}(X)
\end{aligned}
$$

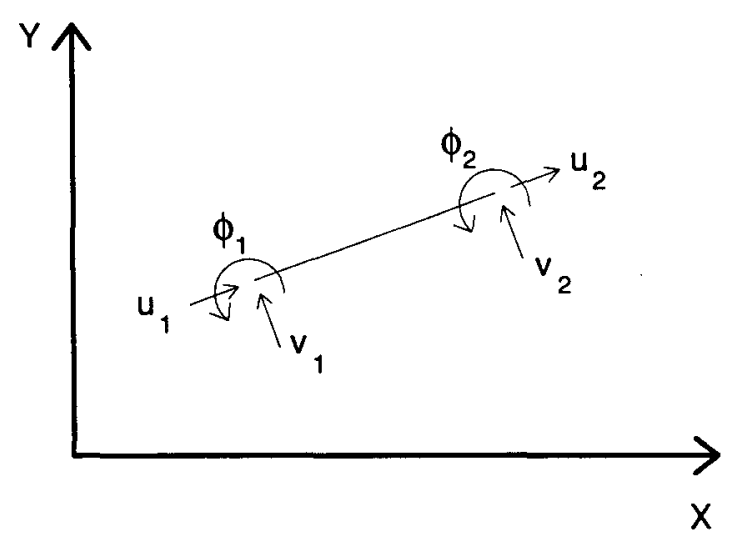

FIGURA 3.2- Elemento finito de pórtico plano 


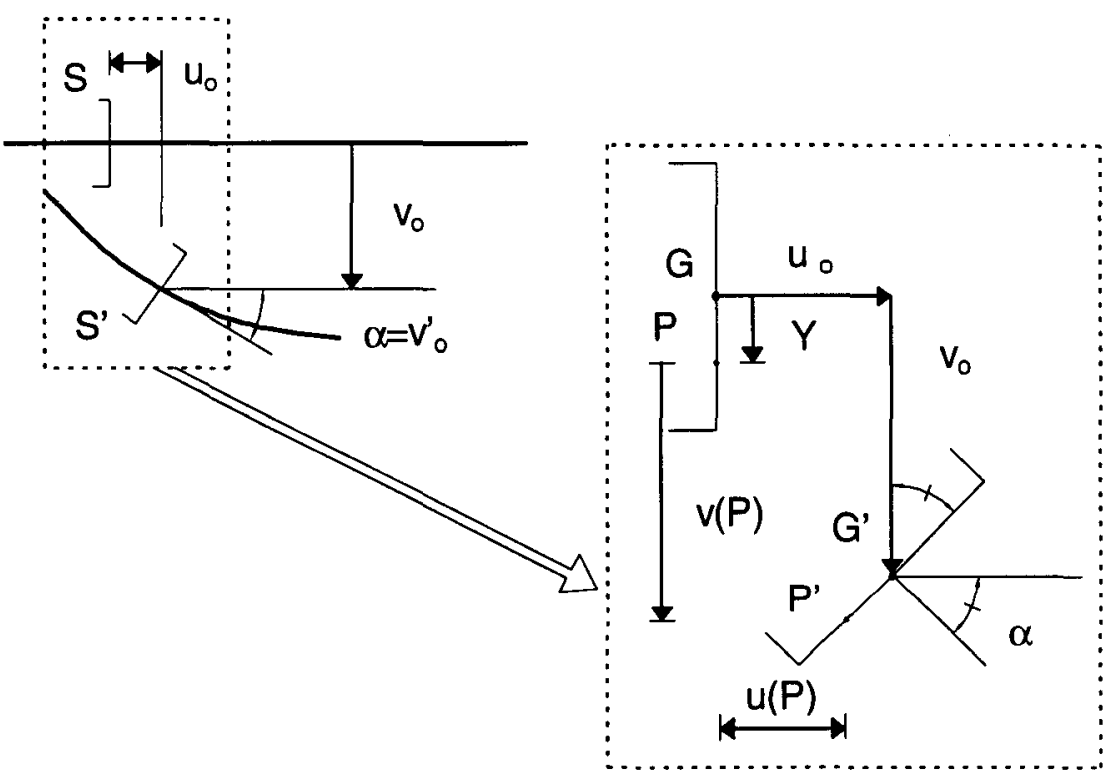

FIGURA 3.3- Campo de deslocamentos na flexão - adaptada de MAZZILLI (1988)

A aproximação adotada para o campo dos deslocamentos consiste em admitir que o deslocamento axial é linear, o transversal tem variação cúbica e a rotação da seção é obtida da derivada primeira do deslocamento transversal. As funções de interpolação usadas são, portanto, dadas por:

$$
\begin{aligned}
& N_{1}(X)=\left(1-\frac{X}{L_{0}}\right) \\
& N_{2}(X)=1-3 \cdot\left(\frac{X}{L_{0}}\right)^{2}+2 \cdot\left(\frac{X}{L_{0}}\right)^{3} \\
& N_{3}(X)=L_{0} \cdot\left[\left(\frac{X}{L_{0}}\right)-2 \cdot\left(\frac{X}{L_{0}}\right)^{2}+\left(\frac{X}{L_{0}}\right)^{3}\right] \\
& N_{4}(X)=\frac{X}{L_{0}}
\end{aligned}
$$




$$
\begin{aligned}
& N_{5}(X)=3 \cdot\left(\frac{X}{L_{0}}\right)^{2}-2 \cdot\left(\frac{X}{L_{0}}\right)^{3} \\
& N_{6}(X)=L \cdot\left[-\left(\frac{X}{L_{0}}\right)^{2}+\left(\frac{X}{L_{0}}\right)^{3}\right]
\end{aligned}
$$

O vetor de deslocamentos nodais é representado por:

$$
q^{\top}=\left[\begin{array}{llllll}
q_{1} & q_{2} & q_{3} & q_{4} & q_{5} & q_{6}
\end{array}\right]=\left[\begin{array}{llllll}
u_{1} & v_{1} & \phi_{1} & u_{2} & v_{2} & \phi_{2}
\end{array}\right]
$$

Utilizando-se as funções de interpolação descritas e o vetor de deslocamentos nodais, pode-se escrever os deslocamentos $u_{0}(X)$ e $v_{0}(X)$ por:

$$
\begin{aligned}
& u_{0}(X)=\left[\begin{array}{llllll}
N_{1} & 0 & 0 & N_{4} & 0 & 0
\end{array}\right] q \\
& v_{0}(X)=\left[\begin{array}{llllll}
0 & N_{2} & N_{3} & 0 & N_{5} & N_{6}
\end{array}\right] q=G q
\end{aligned}
$$

onde: $\mathrm{G}=\left[\begin{array}{llllll}0 & \mathrm{~N}_{2} & \mathrm{~N}_{3} & 0 & \mathrm{~N}_{5} & \mathrm{~N}_{6}\end{array}\right]$.

Tendo-se em vista a equação (2.6) e as componentes do vetor deslocamento dadas pelas equações (3.27) e (3.28), o tensor de deformação de Green resulta:

$$
\varepsilon=\frac{1}{2}\left[\begin{array}{cc}
2\left(u_{o}^{\prime}-Y v_{o}^{\prime \prime}\right)+\left(u_{o}^{\prime}-Y v_{o}^{\prime \prime}\right)^{2}+\left(v_{o}^{\prime}\right)^{2} & -v_{o}^{\prime}\left(u_{o}^{\prime}-Y v_{o}^{\prime \prime}\right) \\
-v_{o}^{\prime}\left(u_{o}^{\prime}-Y v_{o}^{\prime \prime}\right) & \left(v_{o}^{\prime}\right)^{2}
\end{array}\right]
$$

Coerentemente com a hipótese feita sobre o campo de deslocamentos, podem ser desconsiderados os termos $\varepsilon_{12}$, $\varepsilon_{21}$ e $\varepsilon_{22}$, restando ativa apenas a componente $\varepsilon_{11}$ do tensor de deformação, isto é:

$$
\varepsilon_{11}=\left(u_{0}^{\prime}-Y v_{o}^{\prime \prime}\right)+\frac{1}{2}\left(u_{0}^{\prime}-Y v_{o}^{\prime \prime}\right)^{2}+\frac{1}{2}\left(v_{0}^{\prime}\right)^{2}
$$


Empregando-se as equações (3.29) a (3.34) tem-se que:

$$
\begin{aligned}
& u_{0}^{\prime}=\left[\begin{array}{llllll}
N_{1}^{\prime} & 0 & 0 & N_{4}^{\prime} & 0 & 0
\end{array}\right] q \\
& v_{0}^{\prime}=\left[\begin{array}{llllll}
0 & N_{2}^{\prime} & N_{3}^{\prime} & 0 & N_{5}^{\prime} & N_{6}^{\prime}
\end{array}\right] q \\
& v_{0}^{\prime \prime}=\left[\begin{array}{llllll}
0 & N_{2}^{\prime \prime} & N_{3}^{\prime \prime} & 0 & N_{5}^{\prime \prime} & N_{6}^{\prime \prime}
\end{array}\right] q
\end{aligned}
$$

Por uma questão de simplificação algébrica é conveniente definir os seguintes elementos:

$$
\begin{aligned}
& B=\left[\begin{array}{cccccc}
N_{1}^{\prime} & 0 & 0 & N_{4}^{\prime} & 0 & 0 \\
0 & N_{2}^{\prime \prime} & N_{3}^{\prime \prime} & 0 & N_{5}^{\prime} & N_{6}^{\prime}
\end{array}\right] \\
& H=\left[\begin{array}{cc}
1 & -Y
\end{array}\right] \\
& C=\left[\begin{array}{llllll}
0 & N_{2}^{\prime} & N_{3}^{\prime} & 0 & N_{5}^{\prime} & N_{6}^{\prime}
\end{array}\right]
\end{aligned}
$$

Portanto, utilizando-se as equações (3.43), (3.44) e (3.45) pode-se escrever a deformação $\varepsilon_{11}$ por:

$$
\varepsilon_{11}=H B q+\frac{1}{2}(H B q) \cdot(H B q)+\frac{1}{2}(C q) \cdot(C q)
$$

logo, em notação matricial,

$$
\varepsilon_{11}=H B q+\frac{1}{2} q^{\top} B^{\top} H^{\top} H B q+\frac{1}{2} q^{\top} C^{\top} C q
$$

Empregando-se a equação (3.4a), tem-se que a taxa da deformação de Green é expressa por:

$$
\dot{\varepsilon}_{11}=H B \dot{q}+H B q H B \dot{q}+C q C \dot{q}
$$


Utilizando-se a equação (2.20), a deformação virtual de Green resulta:

$$
\delta \varepsilon_{11}=H B \delta q+q^{\top} B^{\top} H^{\top} H B \delta q+q^{\top} C^{\top} C \delta q
$$

Consequentemente, empregando-se a equação (3.4b) tem-se:

$$
\delta \dot{\varepsilon}_{11}^{\top}=\delta q^{\top}\left(B^{\top} H^{\top} H B+C^{\top} C\right) \dot{q}
$$

Levando-se as equações (3.20), (3.47), (3.48) e (3.49) na equação (3.25) obtém-se a expressão completa da matriz de rigidez tangente para $\circ$ elemento de pórtico plano:

$$
\begin{aligned}
K_{T}= & \int_{V_{0}} B^{\top} H^{\top} D_{11} H B d V_{0}+2 \int_{V_{0}} B^{\top} H^{\top} H B D_{11} q H B d V_{0}+ \\
& +\int_{V_{0}} B^{\top} H^{\top} D_{11} C q C d V_{0}+\int_{V_{0}} B^{\top} H^{\top} H B q D_{11} H B q H B d V_{0}+ \\
& +\int_{V_{0}} B^{\top} H^{\top} H B q D_{11} C q C d V_{0}+\int_{V_{0}} C^{\top} C_{q D} D_{11} H B d V_{0}+ \\
& +\int_{V_{0}} C^{\top} C_{q D_{11}} H B q H B d V_{0}+\int_{V_{0}} C^{\top} C_{q D_{11}} C q C d V_{0}+ \\
& +\int_{V_{0}} B^{\top} H^{\top} S_{11} H B d V_{0}+\int_{V_{0}} C^{\top} S_{11} C d V_{0}
\end{aligned}
$$

\subsubsection{1- APROXIMAÇÃO NA MATRIZ DE RIGIDEZ TANGENTE}

Seguindo-se as observações feitas em MAZZILLI (1988), pode-se fazer uma aproximação na expressão da matriz de rigidez tangente, equação (3.50). Desprezando-se os termos relativos a $\left(u^{\prime}\right)^{3},\left(u^{\prime}\right)^{2}\left(v^{\prime}\right)^{2}$ e $\left(u^{\prime}\right)^{4}$ (onde $u^{\prime}=\mathrm{HBq}$ e $v^{\prime}=C q$ ), a expressão da matriz de rigidez tangente passa a ser dada por: 


$$
\begin{aligned}
K_{T}= & \int_{V_{0}} B^{T} H^{\top} D_{11} H B d V_{0}+\int_{V_{0}} B^{\top} H^{\top} D_{11} C q C d V_{0}+ \\
& +\int_{V_{0}} C^{T} C q D_{11} H B d V_{0}+\int_{V_{0}} C^{T} C_{q} D_{11} C q C d V_{0}+ \\
& +\int_{V_{0}} B^{\top} H^{\top} S_{11} H B d V_{0}+\int_{V_{0}} C^{\top} S_{11} C d V_{0}
\end{aligned}
$$

Efetuando-se as integrais descritas na expressão (3.51) com relação à configuração inicial obtêm-se as matrizes que compõem a matriz de rigidez tangente para o elemento finito de pórtico plano, que pode ser escrita numa forma aditiva por:

$$
\mathrm{K}_{\mathrm{T}}=\mathrm{K}_{1}+\mathrm{K}_{2}+\mathrm{K}_{3}+\mathrm{K}_{4}+\mathrm{K}_{5}+\mathrm{K}_{6}
$$

Onde: $\mathrm{K}_{0}=\mathrm{K}_{1}, \mathrm{~K}_{\mathrm{L}}=\mathrm{K}_{2}+\mathrm{K}_{3}+\mathrm{K}_{4}$ e $\mathrm{K}_{\sigma}=\mathrm{K}_{5}+\mathrm{K}_{6}$.

Utilizando-se o "software Mathcad 5.0" para o cálculo das integrais da expressão (3.51), tem-se que as parcelas de $K_{T}$ são dadas por:

$$
\int_{V_{0}} B^{\top} H^{\top} D_{11} H B d V_{0}=K_{1}
$$

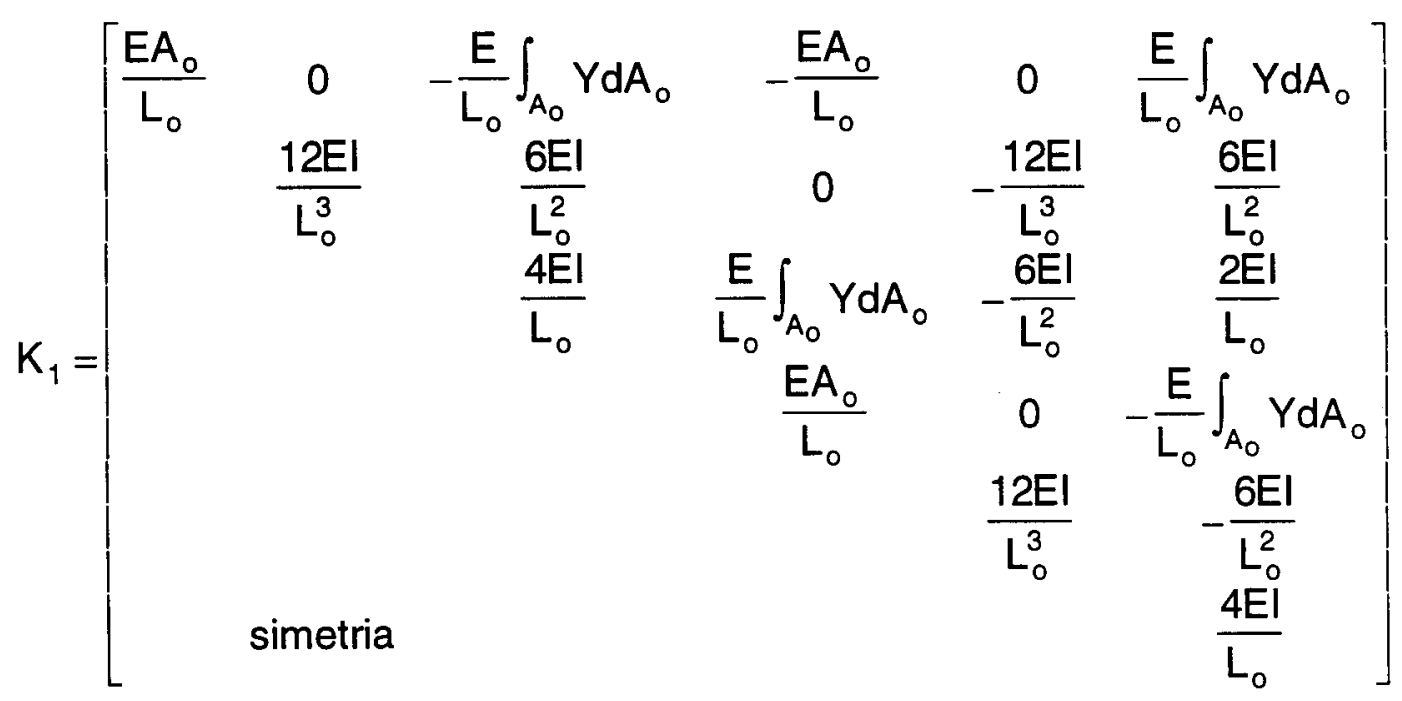




$$
\int_{V_{0}} B^{\top} H^{\top} D_{11} C q C d V_{0}=K_{2}
$$

$$
K_{2}=\left[\begin{array}{llllll}
R_{11} & R_{12} & R_{13} & R_{14} & R_{15} & R_{16} \\
R_{21} & R_{22} & R_{23} & R_{24} & R_{25} & R_{26} \\
R_{31} & R_{32} & R_{33} & R_{34} & R_{35} & R_{36} \\
R_{41} & R_{42} & R_{43} & R_{44} & R_{45} & R_{46} \\
R_{51} & R_{52} & R_{53} & R_{54} & R_{55} & R_{56} \\
R_{61} & R_{62} & R_{63} & R_{64} & R_{65} & R_{66}
\end{array}\right]
$$

onde:

$$
\begin{aligned}
& R_{i 1}=R_{i 4}=0 ; i=1, \ldots, 6 \\
& R_{12}=-\frac{E A_{\circ}}{10 L_{\circ}^{2}}\left(12 q_{2}+q_{3} L_{\circ}-12 q_{5}+q_{6} L_{\circ}\right) \\
& R_{13}=-\frac{E A_{\circ}}{30 L_{\circ}}\left(3 q_{2}+4 q_{3} L_{\circ}-3 q_{5}-q_{6} L_{\circ}\right) \\
& R_{15}=\frac{E A_{\circ}}{10 L_{o}^{2}}\left(12 q_{2}+q_{3} L_{\circ}-12 q_{5}+q_{6} L_{\circ}\right) \\
& R_{16}=\frac{E A_{\circ}}{30 L_{\circ}}\left(-3 q_{2}+q_{3} L_{\circ}+3 q_{5}-4 q_{6} L_{\circ}\right) \\
& R_{22}=-\frac{3 E}{5 L_{\circ}^{2}}\left(q_{3}-q_{6}\right) \int_{A 0} Y d A_{\circ} \\
& R_{23}=\frac{E}{5 L_{o}^{2}}\left(-3 q_{2}+2 q_{3} L_{\circ}+3 q_{5}\right) \int_{A o} Y d A_{\circ} \\
& R_{25}=\frac{3 E}{5 L_{o}^{2}}\left(q_{3}-q_{6}\right) \int_{A o} Y d A_{\circ}
\end{aligned}
$$


32

$$
\begin{aligned}
& R_{26}=-\frac{E}{5 L_{o}^{2}}\left(-3 q_{2}+3 q_{5}+2 q_{6} L_{\circ}\right) \int_{A 0} Y d A_{\circ} \\
& R_{32}=-\frac{E}{5 L_{0}^{2}}\left(-6 q_{2}+q_{3} L_{\circ}+6 q_{5}-2 q_{6} L_{0}\right) \int_{A 0} Y d A_{\circ} \\
& R_{33}=\frac{E}{30 L_{0}}\left(-6 q_{2}+10 q_{3} L_{\circ}+6 q_{5}-q_{6} L_{0}\right) \int_{A 0} Y d A_{0} \\
& R_{35}=\frac{E}{5 L_{o}^{2}}\left(-6 q_{2}+q_{3} L_{\circ}+6 q_{5}-2 q_{6} L_{\circ}\right) \int_{A 0} Y d A_{\circ} \\
& R_{36}=-\frac{E}{30 L_{0}}\left(-12 q_{2}+q_{3} L_{\circ}+12 q_{5}+2 q_{6} L_{0}\right) \int_{A 0} Y d A_{0} \\
& R_{42}=\frac{E A_{\circ}}{10 L_{\circ}^{2}}\left(12 q_{2}+q_{3} L_{\circ}-12 q_{5}+q_{6} L_{0}\right) \\
& R_{43}=\frac{E A_{0}}{30 L_{\circ}}\left(3 q_{2}+4 q_{3} L_{\circ}-3 q_{5}-q_{6} L_{\circ}\right) \\
& R_{45}=-\frac{E A_{o}}{10 L_{o}^{2}}\left(12 q_{2}+q_{3} L_{\circ}-12 q_{5}+q_{6} L_{0}\right) \\
& R_{46}=-\frac{E A_{\circ}}{30 L_{0}}\left(-3 q_{2}+q_{3} L_{\circ}+3 q_{5}-4 q_{6} L_{\circ}\right) \\
& R_{52}=\frac{3 E}{5 L_{0}^{2}}\left(q_{3}-q_{6}\right) \int_{A 0} Y d A_{\circ} \\
& R_{53}=-\frac{E}{5 L_{o}^{2}}\left(-3 q_{2}+2 q_{3} L_{\circ}+3 q_{5}\right) \int_{A 0} Y d A_{\circ} \\
& R_{55}=-\frac{3 E}{5 L_{0}^{2}}\left(q_{3}-q_{6}\right) \int_{A 0} Y d A_{0}
\end{aligned}
$$




$$
\begin{aligned}
& R_{56}=\frac{E}{5 L_{\circ}^{2}}\left(-3 q_{2}+3 q_{5}+2 q_{6} L_{0}\right) \int_{A o} Y d A_{\circ} \\
& R_{62}=-\frac{E}{5 L_{o}^{2}}\left(6 q_{2}+2 q_{3} L_{\circ}-6 q_{5}-q_{6} L_{\circ}\right) \int_{A o} Y d A_{o} \\
& R_{63}=\frac{E}{30 L_{o}}\left(-12 q_{2}+2 q_{3} L_{\circ}+12 q_{5}+q_{6} L_{o}\right) \int_{A o} Y d A_{\circ} \\
& R_{65}=\frac{E}{5 L_{\circ}^{2}}\left(6 q_{2}+2 q_{3} L_{\circ}-6 q_{5}-q_{6} L_{o}\right) \int_{A o} Y d A_{\circ} \\
& R_{66}=\frac{E}{30 L_{o}}\left(6 q_{2}+q_{3} L_{o}-6 q_{5}-10 q_{6} L_{\circ}\right) \int_{A o} Y d A_{\circ}
\end{aligned}
$$

$\int_{V_{0}} C^{\top} C q D_{11} H B d V_{o}=K_{3}$

$$
\begin{gathered}
\mathrm{K}_{3}=\mathrm{K}_{2}^{\top} \\
\int_{\mathrm{V}_{0}} \mathrm{C}^{\top} \mathrm{CqD} \mathrm{I}_{11} \mathrm{CqC} d \mathrm{~V}_{0}=\mathrm{K}_{4}
\end{gathered}
$$

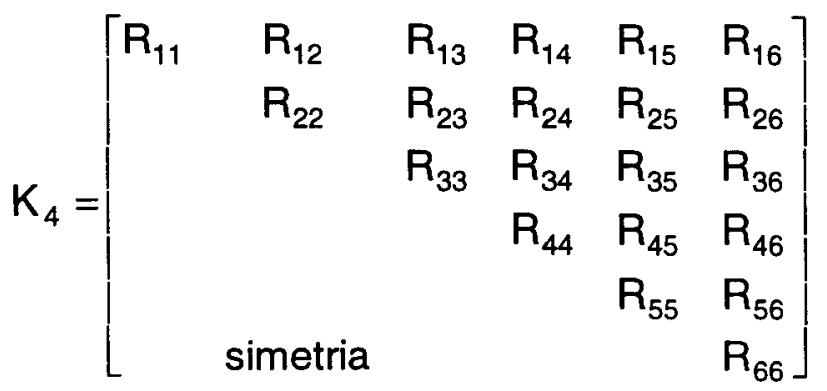

$$
R_{1 i}=0 ; i=1, \ldots, 6 \quad R_{4 i}=0 ; i=4, \ldots, 6
$$




$$
\begin{aligned}
& R_{22}=\frac{3 E A_{\circ}}{35 L_{0}^{3}}\left(\begin{array}{l}
-6 q_{3} q_{5} L_{\circ}-6 q_{5} q_{6} L_{\circ}+q_{6}^{2} L_{\circ}^{2}+24 q_{2}^{2}+6 q_{2} q_{6} L_{\circ}+ \\
+24 q_{5}^{2}+q_{3}^{2} L_{0}^{2}+6 q_{3} q_{2} L_{\circ}-48 q_{5} q_{2}
\end{array}\right) \\
& R_{23}=-\frac{E A_{o}}{140 L_{0}^{2}}\left(\begin{array}{l}
-24 q_{2} q_{3} L_{o}+72 q_{2} q_{5}-2 q_{3} q_{6} L_{o}^{2}+q_{3}^{2} L_{o}^{2}+ \\
+24 q_{3} q_{5} L_{o}-36 q_{5}^{2}-36 q_{2}^{2}-q_{6}^{2} L_{o}^{2}
\end{array}\right) \\
& R_{25}=-\frac{3 E A_{\circ}}{35 L_{0}^{3}}\left(\begin{array}{l}
-6 q_{3} q_{5} L_{\circ}-6 q_{5} q_{6} L_{0}+q_{6}^{2} L_{o}^{2}+24 q_{2}^{2}+6 q_{2} q_{6} L_{o}+ \\
+24 q_{5}^{2}+q_{3}^{2} L_{o}^{2}+6 q_{3} q_{2} L_{\circ}-48 q_{5} q_{2}
\end{array}\right) \\
& R_{26}=\frac{E A_{\circ}}{140 L_{o}^{2}}\left(\begin{array}{l}
24 q_{2} q_{6} L_{o}+36 q_{2}^{2}+q_{3}^{2} L_{o}^{2}-72 q_{2} q_{5}-q_{6}^{2} L_{o}^{2}+ \\
+2 q_{3} q_{6} L_{o}^{2}-24 q_{5} q_{6} L_{\circ}+36 q_{5}^{2}
\end{array}\right) \\
& R_{33}=\frac{E A_{0}}{210 L_{0}}\left(\begin{array}{l}
18 q_{2}^{2}-3 q_{5} q_{6} L_{\circ}+3 q_{2} q_{6} L_{\circ}+3 q_{3} q_{5} L_{\circ}-3 q_{3} q_{6} L_{\circ}^{2}+ \\
-36 q_{2} q_{5}+12 q_{3}^{2} L_{0}^{2}+q_{6}^{2} L_{0}^{2}-3 q_{2} q_{3} L_{0}+18 q_{5}^{2}
\end{array}\right) \\
& R_{35}=\frac{E A_{0}}{140 L_{0}^{2}}\left(\begin{array}{l}
-24 q_{2} q_{3} L_{0}+72 q_{2} q_{5}-2 q_{3} q_{6} L_{o}^{2}+q_{3}^{2} L_{o}^{2}+ \\
+24 q_{3} q_{5} L_{o}-36 q_{5}^{2}-36 q_{2}^{2}-q_{6}^{2} L_{o}^{2}
\end{array}\right) \\
& R_{36}=E A_{\circ}\left(\begin{array}{l}
-\frac{1}{140} q_{3}^{2} L_{\circ}-\frac{1}{140} q_{6}^{2} L_{\circ}+\frac{1}{70} q_{2} q_{3}-\frac{1}{70} q_{3} q_{5}+ \\
+\frac{1}{105} q_{3} q_{6} L_{\circ}+\frac{1}{70} q_{2} q_{6}-\frac{1}{70} q_{5} q_{6}
\end{array}\right) \\
& R_{55}=\frac{3 E A_{\circ}}{35 L_{0}^{3}}\left(\begin{array}{l}
-6 q_{3} q_{5} L_{\circ}-6 q_{5} q_{6} L_{\circ}+q_{6}^{2} L_{o}^{2}+24 q_{2}^{2}+6 q_{2} q_{6} L_{\circ}+ \\
+24 q_{5}^{2}+q_{3}^{2} L_{0}^{2}+6 q_{3} q_{2} L_{\circ}-48 q_{5} q_{2}
\end{array}\right) \\
& R_{56}=-\frac{E A_{0}}{140 L_{o}^{2}}\left(\begin{array}{l}
24 q_{2} q_{6} L_{0}+36 q_{2}^{2}+q_{3}^{2} L_{o}^{2}-72 q_{2} q_{5}-q_{6}^{2} L_{o}^{2}+ \\
+2 q_{3} q_{6} L_{o}^{2}-24 q_{5} q_{6} L_{o}+36 q_{5}^{2}
\end{array}\right) \\
& R_{66}=\frac{E A_{0}}{210 L_{0}}\left(\begin{array}{l}
-3 q_{2} q_{6} L_{\circ}+12 q_{6}^{2} L_{0}^{2}+3 q_{2} q_{3} L_{\circ}-36 q_{2} q_{5}+18 q_{2}^{2}+ \\
+q_{3}^{2} L_{0}^{2}-3 q_{3} q_{5} L_{0}+3 q_{5} q_{6} L_{0}+18 q_{5}^{2}-3 q_{3} q_{6} L_{0}^{2}
\end{array}\right)
\end{aligned}
$$


$\int_{V_{0}} B^{\top} H^{\top} S_{11} H B d V_{0}=K_{5}$

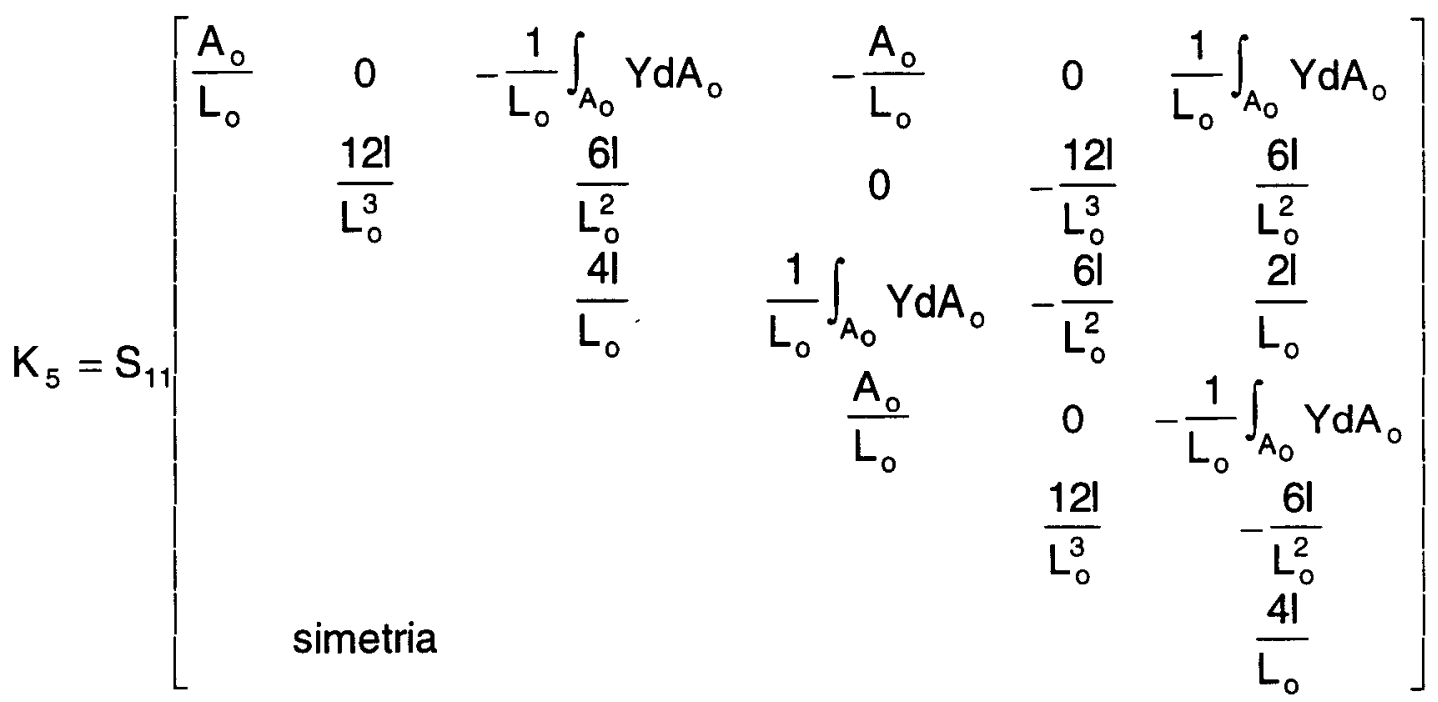

$\int_{V_{0}} C^{\top} S_{11} C d V_{o}=K_{6}$

$\mathrm{K}_{6}=N\left[\begin{array}{cccccc}0 & 0 & 0 & 0 & 0 & 0 \\ & \frac{6}{5 \mathrm{~L}_{0}} & \frac{1}{10} & 0 & -\frac{6}{5 \mathrm{~L}_{o}} & \frac{1}{10} \\ & & \frac{2 \mathrm{~L}_{\circ}}{15} & 0 & -\frac{1}{10} & -\frac{\mathrm{L}_{\circ}}{30} \\ & & & 0 & 0 & 0 \\ & & & & \frac{6}{5 \mathrm{~L}_{\circ}} & -\frac{1}{10} \\ \text { simetria } & & & & \frac{2 \mathrm{~L}_{\circ}}{15}\end{array}\right]$

Como a origem do sistema de referência coincide com o centróide da seção o momento estático é nulo, isto é $\int_{A_{o}} Y d A_{\circ}=0$. 
Para o caso do elemento finito de pórtico plano as considerações sobre o campo dos deslocamentos e aproximações na expressão da matriz de rigidez tangente foram feitas baseando-se no trabalho de MAZZILLI (1988).

Neste caso a deformação de Green pode ser escrita:

$$
\varepsilon=\frac{\partial u}{\partial X}+\frac{1}{2}\left(\frac{\partial v}{\partial X}\right)^{2}+\frac{1}{2}\left(\frac{\partial u}{\partial X}\right)^{2}
$$

onde: $u(X, Y)=u_{0}(X)-Y v_{o}^{\prime}$ e $v(X, Y)=v_{0}(X)$.

Segundo MAZZILLI (1988), em diversos trabalhos relacionados com este assunto a última parcela é desprezada supondo-a muito menor que as outras; porém, esta aproximação às vezes pode conduzir a erros não desprezíveis na matriz de rigidez.

\subsubsection{ELEMENTO FINITO PARA ANÁLISE BIDIMENSIONAL}

Para o elemento finito de estado plano será apresentada a expressão da matriz de rigidez tangente, empregando-se um procedimento análogo ao apresentado por WRIGGERS (1990).

Admite-se que a estrutura esteja discretizada por elementos finitos isoparamétricos, na configuração inicial. A geometria $X$, o campo dos deslocamentos $u$, o campo dos deslocamentos virtuais $\delta u$ e o campo das velocidades nodais ù são interpolados dentro de um elemento finito $\Omega_{e}$ pelas mesmas funções de forma.

Para o elemento finito empregado na análise bidimensional, representado na figura 3.4 , valem: 


$$
\begin{array}{ll}
X_{e}=\sum_{i=1}^{n} N_{i} X_{i} & \delta u_{e}=\sum_{i=1}^{n} N_{i} \delta q_{i} \\
u_{e}=\sum_{i=1}^{n} N_{i} q_{i} & \dot{u}_{e}=\sum_{i=1}^{n} N_{i} \dot{q}_{i}
\end{array}
$$

Onde $N_{i}$ são as funções de forma, $n$ é o número de nós associados a cada elemento.

Não será mostrado aqui o caminho para geração das funções de forma, que pode ser facilmente encontrado em livros sobre método dos elementos finitos, ZIENKIEWICZ (1989) e BATHE (1982).
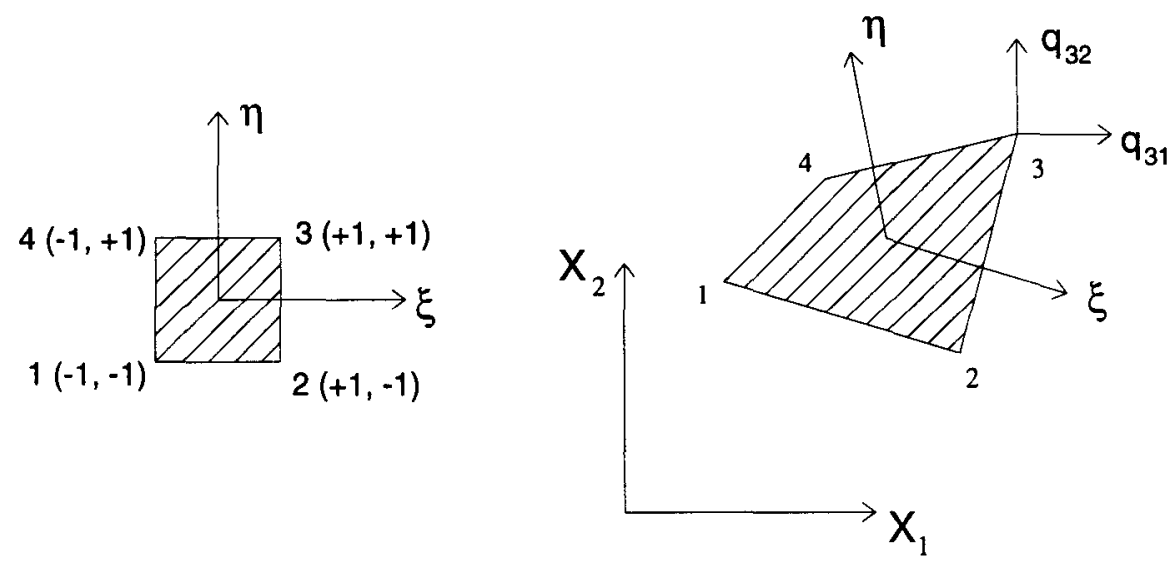

FIGURA 3.4- Elemento finito para análise bidimensional em coordenadas adimensionais - WRIGGERS (1990)

Sabendo-se que $u_{e}=\sum_{i=1}^{n} N_{i} q_{i}$, para o elemento finito da figura 3.4 tem-se $u_{e}=N_{1} q_{1}+N_{2} q_{2}+N_{3} q_{3}+N_{4} q_{4}$. Porém o vetor deslocamento possui 
componentes na direção $X_{1}$ e $X_{2}$, isto é $\left\{u_{e}\right\}=\left\{\begin{array}{l}u_{1} \\ u_{2}\end{array}\right\}$ e do mesmo modo os deslocamentos nodais $q_{i},\left\{q_{i}\right\}=\left\{\begin{array}{l}q_{1 i} \\ q_{2 i}\end{array}\right\}$.

Assim, por exemplo $\left\{q_{1}\right\}=\left\{\begin{array}{l}q_{11} \\ q_{21}\end{array}\right\}$.

Portanto, reescrevendo-se o vetor $u$ em componentes, tem-se que:

$$
\left\{\begin{array}{l}
u_{1} \\
u_{2}
\end{array}\right\}=\left\{\begin{array}{l}
N_{1} q_{11}+N_{2} q_{12}+N_{3} q_{13}+N_{4} q_{14} \\
N_{1} q_{21}+N_{2} q_{22}+N_{3} q_{23}+N_{4} q_{24}
\end{array}\right\}
$$

logo, as componentes do vetor deslocamento podem ser escritas por:

$$
u_{\alpha}=\sum_{i=1}^{n} N_{i} q_{\alpha i} \operatorname{com} \alpha=1,2
$$

Deste modo, as derivadas parciais das componentes do deslocamento com relação a $\beta$, podem ser escritas de maneira genérica por:

$$
u_{\alpha, \beta}=\sum_{i=1}^{n} N_{i, \beta} q_{\alpha i}
$$

Dentro do conceito isoparamétrico, para calcular as variações utilizadas na equação de equilíbrio, a regra da cadeia deve ser usada para o cálculo da derivada parcial de $\mathrm{N}_{\mathrm{i}}$ em relação à $\mathrm{X}_{\mathrm{A}}$, tal como:

$$
\left\{\begin{array}{l}
N_{i, 1} \\
N_{i, 2}
\end{array}\right\}=\frac{1}{\operatorname{det} J_{c}}\left[\begin{array}{cc}
X_{2, \eta} & -X_{2, \xi} \\
-X_{1, \eta} & X_{1, \xi}
\end{array}\right]\left[\begin{array}{l}
N_{i, \xi} \\
N_{i, \eta}
\end{array}\right]
$$

Como as funções de forma são definidas num sistema de coordenadas locais $\xi, \eta$, uma transformação para as coordenadas globais 
$X_{1}, X_{2}$ é necessária. Na equação (3.56) det $J_{c}$ é o determinante da transformação Jacobiana entre $d \xi$, $d \eta$ e $d X_{1}, d X_{2}$.

Onde:

$$
J_{c}=\left[\begin{array}{ll}
X_{1, \xi} & X_{2, \xi} \\
X_{1, \eta} & X_{2, \eta}
\end{array}\right]
$$

Por outro lado, sabe-se que a configuração atual pode ser escrita em termos da configuração de referência por $x=X+u_{e}$. Então, o tensor de deformação de Green para problemas de pequenas deformações pode ser escrito empregando-se o gradiente dos deslocamentos, que é dado por:

$$
\nabla u_{e}=\left[\begin{array}{ll}
u_{1,1} & u_{1,2} \\
u_{2,1} & u_{2,2}
\end{array}\right]
$$

Assim, utilizando-se a equação (3.55) tem-se que a equação (2.6) discretizada para um elemento finito $\Omega_{e}$ pode ser escrita por:

$$
\varepsilon_{e}=\left\{\begin{array}{l}
\varepsilon_{11} \\
\varepsilon_{22} \\
\varepsilon_{12}
\end{array}\right\}=\sum_{i=1}^{n}\left[\begin{array}{cc}
N_{i, 1}+\frac{1}{2} u_{1,1} N_{i, 1} & \frac{1}{2} u_{2,1} N_{i, 1} \\
\frac{1}{2} u_{1,2} N_{i, 2} & N_{i, 2}+\frac{1}{2} u_{2,2} N_{i, 2} \\
N_{i, 2}+\frac{1}{2}\left(u_{1,1} N_{i, 2}+u_{1,2} N_{i, 1}\right) & N_{i, 1}+\frac{1}{2}\left(u_{2,2} N_{i, 1}+u_{2,1} N_{i, 2}\right)
\end{array}\right\}\left\{\begin{array}{l}
\left.q_{1}\right\}_{q_{2}}
\end{array}\right\}_{i}
$$

Definindo-se:

$$
B_{0}=\left[\begin{array}{cc}
N_{i, 1} & 0 \\
0 & N_{i, 2} \\
N_{i, 2} & N_{i, 1}
\end{array}\right] \text { e } B_{L}=\left[\begin{array}{cc}
u_{1,1} N_{i, 1} & u_{2,1} N_{i, 1} \\
u_{1,2} N_{i, 2} & u_{2,2} N_{i, 2} \\
\left(u_{1,1} N_{i, 2}+u_{1,2} N_{i, 1}\right) & \left(u_{2,2} N_{i, 1}+u_{2,1} N_{i, 2}\right)
\end{array}\right]
$$

a equação (3.58) pode ser reescrita por: 


$$
\varepsilon_{e}=\sum_{i=1}^{n}\left[B_{o i}+\frac{1}{2} B_{L i}\left(q_{i}\right)\right] q_{i}
$$

Utilizando-se a equação (2.20), a deformação virtual de Green em forma matricial resulta:

$$
\delta \varepsilon_{e}=\sum_{i=1}^{n}\left[B_{o i}+B_{L i}\left(q_{i}\right)\right] \delta q_{i}
$$

A taxa de deformação de Green neste caso, é dada por:

$$
\dot{\varepsilon}_{e}=\sum_{i=1}^{n}\left[B_{\circ i}+B_{L i}\left(q_{i}\right)\right] \dot{q}_{i}
$$

e a taxa de deformação virtual por:

$$
\delta \dot{\varepsilon}_{e}=\sum_{i=1}^{n} \sum_{k=1}^{n}\left[\begin{array}{l}
N_{i, 1} \\
N_{i, 2}
\end{array}\right] \dot{q}_{i}^{\top}\left[\begin{array}{ll}
N k_{i, 1} & N_{k, 2}
\end{array}\right] \delta q_{k}
$$

Lembrando que pelo PTV o equilibrio é expresso por:

$$
R_{e}(q, \delta u)=\int_{V_{o}} S \cdot \delta \varepsilon_{e} d V_{o}-\int_{V_{o}} \delta u_{e} \cdot \rho b d V_{o}-\int_{A_{o}} \delta u_{e} \cdot \hat{t} d A_{o}=0
$$

substituem-se as equações (3.53) e (3.62) na equação (3.65), de modo a resultar a forma discretizada para análise bidimensional:

$$
R_{e}(q, \delta q)=\sum_{i=1}^{n} \delta q_{i}^{\top}\left\{\int_{\Omega_{e}}\left(B_{o i}+B_{L i}\right)^{\top} S_{i} d \Omega_{e}-\int_{\Omega_{e}} N_{i} \rho b d \Omega_{e}-\int_{\partial \Omega_{e}} N_{i} \hat{t} d \partial \Omega_{e}\right\}=0
$$

$$
\operatorname{com} S^{\top}=\left\{S_{11}, S_{22}, S_{12}\right\} \text { e } D=\left[\begin{array}{lll}
D_{11} & D_{12} & D_{13} \\
D_{21} & D_{22} & D_{23} \\
D_{31} & D_{32} & D_{33}
\end{array}\right]
$$


Como visto em seções anteriores, a equação do PTV não é linear nos deslocamentos e a sua linearização leva à expressão da matriz de rigidez tangente. Portanto, com objetivo de explicitar uma expressão para a rigidez tangente, da equação do PTV desenvolvida em $1^{\underline{a}}$ ordem no tempo $e$ desconsiderando-se forças externas, tem-se:

$$
\frac{\mathrm{d}}{\mathrm{dt}} \mathrm{R}_{e}(q, \delta q)=\int_{\Omega_{e}} \delta \varepsilon_{e}^{\top} \dot{S} \mathrm{~d} \Omega_{e}+\int_{\Omega_{e}} \delta \dot{\varepsilon}_{e}^{\top}|\hat{S}| d \Omega_{e}
$$

Substituindo-se as equações (3.62), (3.20) e (3.63) na equação (3.67) resulta:

$$
\frac{d}{d t} R_{e}\left(q, \delta q_{i}\right) \dot{q}_{i}=\sum_{i=1 k=1}^{n} \sum_{k=1}^{n} \delta q_{i}^{\top} \int_{\Omega_{e}}\left[\left(B_{o i}+B_{L i}\right)^{\top} D\left(B_{o k}+B_{L k}\right)+G_{i} G_{k}^{\top} \mid \hat{S} \|\right] d \Omega_{e} \dot{q}_{i}=0
$$

onde $G_{i}$ e $|\hat{S}|$ são definidas por:

$$
G_{i}=\left[\begin{array}{l}
N_{k, 1} \\
N_{k, 2}
\end{array}\right] \quad|\hat{S}|=\left[\begin{array}{ll}
S_{11} & S_{12} \\
S_{21} & S_{22}
\end{array}\right]
$$

Definindo-se, finalmente, a matriz $B_{i}=B_{o i}+B_{L i}$ em coordenadas locais, a expressão da matriz de rigidez tangente é:

$$
K_{T_{e}}=\sum_{i=1}^{n} \sum_{k=1}^{n} \int_{\Omega_{e}}\left[B_{i}^{\top} D B_{k}+G_{i} G_{k}^{T}|\hat{s}|\right] d \Omega_{e}
$$

\subsubsection{FORMULAÇÃO LAGRANGIANA ATUALIZADA}

Sabe-se que as equações de equilíbrio na posição deslocada não são lineares e uma outra maneira de se encontrar a solução consiste em empregar a forma linearizada em combinação com uma atualização contínua da configuração de referência, em intervalos de tempo muito 
pequenos. Essa atualização é feita de modo que, ao se atingir o equilíbrio, a configuração de referência atualizada praticamente coincide com a configuração deformada final, ver figura 3.5.

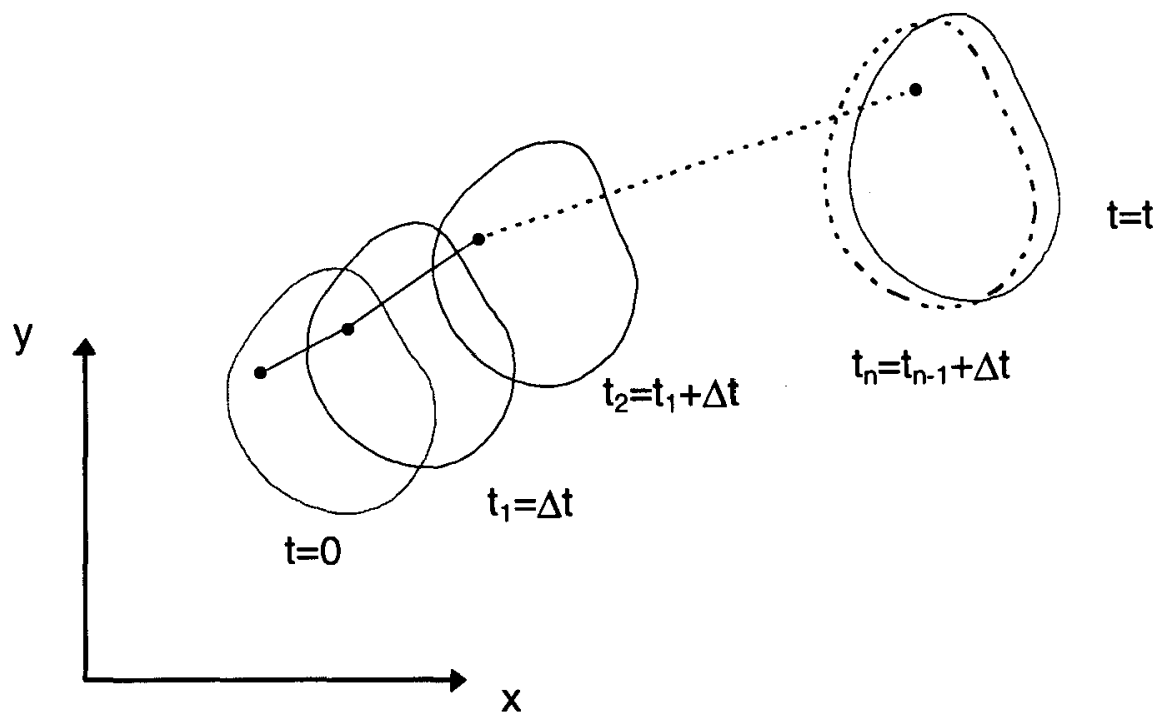

FIGURA 3.5- Atualização da configuração de referência

Assim, a formulação Lagrangiana Atualizada é introduzida de modo que as equações incrementais de equilibrio são formalmente escritas de maneira análoga às da formulação Lagrangiana Total. A diferença, portanto, entre a forma Lagrangiana Atualizada e a Lagrangiana Total é a configuração tomada como referência

Deste modo, na formulação Lagrangiana Atualizada utilizam-se a deformação de Green e a tensão de Piola-Kirchhoff de $2^{2}$ espécie, porém escritos na configuração equilibrada correspondente ao passo de tempo anterior. Logo, os tensores de deformação e taxa de deformação de Green em coordenadas atualizadas passam a ser dados por:

$$
\varepsilon^{*}=\frac{1}{2}\left[\left(\frac{\partial u}{\partial x}\right)+\left(\frac{\partial u}{\partial x}\right)^{\top}+\left(\frac{\partial u}{\partial x}\right)^{\top}\left(\frac{\partial u}{\partial x}\right)\right]
$$




$$
\dot{\varepsilon}^{*}=\frac{1}{2}\left[\left(\frac{\partial \dot{u}}{\partial x}\right)+\left(\frac{\partial \dot{u}}{\partial x}\right)^{\top}+\left(\frac{\partial \dot{u}}{\partial x}\right)^{\top}\left(\frac{\partial u}{\partial x}\right)+\left(\frac{\partial u}{\partial x}\right)^{\top}\left(\frac{\partial \dot{u}}{\partial x}\right)\right]
$$

Em consequência, o tensor de deformação virtual de Green e o tensor taxa de deformação virtual respectivamente resultam:

$$
\begin{gathered}
\delta \varepsilon^{*}=\frac{1}{2}\left[\left(\frac{\partial \delta \mathrm{u}}{\partial \mathrm{x}}\right)+\left(\frac{\partial \delta \mathrm{u}}{\partial \mathrm{x}}\right)^{\top}+\left(\frac{\partial \delta \mathrm{u}}{\partial \mathrm{x}}\right)^{\top}\left(\frac{\partial \mathrm{u}}{\partial \mathrm{x}}\right)+\left(\frac{\partial \mathrm{u}}{\partial \mathrm{x}}\right)^{\top}\left(\frac{\partial \delta \mathrm{u}}{\partial \mathrm{x}}\right)\right] \\
\delta \dot{\varepsilon}^{*}=\left(\frac{\partial \delta \mathrm{u}}{\partial \mathrm{x}}\right)^{\top}\left(\frac{\partial \dot{u}}{\partial \mathrm{x}}\right)
\end{gathered}
$$

A relação constitutiva em termos de taxas para pequenas deformações é representada por:

$$
\left\{\dot{S}^{*}\right\}=D\left\{\dot{\varepsilon}^{*}\right\}
$$

Nas situações em que o intervalo de tempo de uma configuração à outra é muito pequeno, pode-se considerar apenas a parte linear na deformação virtual e na taxa da deformação de Green.

Do ponto de vista de busca de uma solução aproximada, no caso de se empregarem os mesmos elementos finitos utilizados na formulação Lagrangiana Total, os deslocamentos são interpolados de maneira análoga, porém com relação à última configuração equilibrada.

\subsubsection{ELEMENTO FINITO DE TRELIÇA PLANA}

Considere-se o elemento finito de treliça plana utilizado na formulação Lagrangiana Total, porém em coordenadas locais atualizadas. 
Assim, utilizando-se apenas a parte linear do tensor de deformação virtual na configuração atualizada, tem-se que a deformação virtual de Green é escrita por:

$$
\delta \varepsilon^{*}=B_{o}^{*} \delta q
$$

Onde $B_{0}^{*}=\left[\begin{array}{llll}-\frac{1}{L} & 0 & \frac{1}{L} & 0\end{array}\right]$ e $L$ é o comprimento na configuração atualizada.

A equação de equilíbrio correspondente à descrição em questão para o elemento finito de treliça plana é:

$$
r=\int_{V} B^{* \top}\{\sigma\} d V-f_{b}-f_{\bar{t}}
$$

onde $B^{*}=B_{0}^{*}$.

Linearizando-se essa equação resulta:

$$
\left(\int_{V} r \cdot \delta u d V\right)_{t}+\frac{d}{d t}\left(\int_{V} r \cdot \delta u d V\right)_{t} \Delta t=0
$$

ou

$$
(\mathrm{r})_{\mathrm{t}}+\left(\mathrm{K}_{\mathrm{T}} \dot{\mathrm{u}}-\dot{\mathrm{f}}_{\mathrm{b}}-\dot{\mathrm{f}}_{\overline{\mathrm{t}}}\right)_{\mathrm{t}} \Delta \mathrm{t}=0
$$

Fazendo-se uso da equação (3.3) na equação (3.78), obtém-se:

$$
\delta q^{\top} \dot{r}=\delta q^{\top}\left[K_{T} \dot{q}-\dot{f}_{b}-\dot{f}_{t}\right]
$$

portanto, a matriz de rigidez tangente resulta de:

$$
K_{T}=\int_{V}\left(\delta \varepsilon^{*}\right)^{\top} \dot{S}^{*} d V+\int_{V}\left(\delta \dot{\varepsilon}^{*}\right)^{\top} \mid \hat{\alpha} d V
$$


Onde a taxa da componente da deformação de Green é dada por:

$$
\begin{gathered}
\dot{\varepsilon}_{11}^{*}=\frac{\partial \dot{u}_{1}}{\partial x_{1}} \text { ou em notação matricial: } \\
\dot{\varepsilon}_{11}^{*}=B_{\circ}^{*} \dot{q}
\end{gathered}
$$

e a deformação virtual (parte linear) em notação matricial por:

$$
\delta \varepsilon_{11}^{*}=B_{\circ}^{*} \delta q
$$

Por sua vez, utilizando-se a equação (3.74), a taxa da deformação virtual em forma matricial, resulta:

$$
\left(\delta \dot{\varepsilon}_{11}^{*}\right)^{\top}=\delta q^{\top} G^{* \top} G^{*} \dot{q}
$$

onde: $G^{*}=\frac{1}{L}\left[\begin{array}{cccc}-1 & 0 & 1 & 0 \\ 0 & -1 & 0 & 1\end{array}\right]$

Substituindo-se as equações (3.75), (3.81), (3.82) e (3.83) na equação (3.80), a expressão da matriz de rigidez tangente passa a ser escrita na forma:

$$
K_{T}=\int_{V} B^{* \top} D_{11} B^{*} d V+\int_{V} G^{* \top} \sigma_{11} G^{*} d V
$$

Finalmente, efetuando-se as operações indicadas na equação (3.84), a matriz de rigidez tangente para um elemento finito de treliça plana em coordenadas atualizadas, resulta:

$$
\mathrm{K}_{\mathrm{T}}=\mathrm{K}_{\mathrm{o}}+\mathrm{K}_{\sigma}
$$


onde:

$$
K_{0}=\frac{E A}{L}\left[\begin{array}{cccc}
1 & 0 & -1 & 0 \\
0 & 0 & 0 & 0 \\
-1 & 0 & 1 & 0 \\
0 & 0 & 0 & 0
\end{array}\right] \quad K_{\sigma}=\frac{N}{L}\left[\begin{array}{cccc}
1 & 0 & -1 & 0 \\
0 & 1 & 0 & -1 \\
-1 & 0 & 1 & 0 \\
0 & -1 & 0 & 1
\end{array}\right]
$$

\subsubsection{ELEMENTO FINITO DE PÓRTICO PLANO}

Considere-se 0 elemento finito de pórtico plano utilizado na formulação Lagrangiana Total. As hipóteses feitas sobre o campo dos deslocamentos e as funções interpoladoras são as mesmas, porém referemse à última configuração equilibrada.

De maneira análoga à formulação Lagrangiana Total para o elemento de pórtico plano, a componente ativa da deformação é:

$$
\varepsilon_{11}^{*}=\left(u_{o}^{\prime}-y v_{o}^{\prime \prime}\right)+\frac{1}{2}\left(u_{o}^{\prime}-y v_{o}^{\prime \prime}\right)^{2}+\frac{1}{2}\left(v_{o}^{\prime}\right)^{2}
$$

Portanto, utilizando-se as equações (3.43), (3.44) e (3.45), porém em coordenadas atualizadas, a deformação $\varepsilon_{11}^{*}$ (parte linear) pode ser escrita na forma matricial por:

$$
\varepsilon_{11}^{*}=H^{*} B^{*} q
$$

onde a matriz $\mathrm{B}^{*}$ refere-se à configuração atualizada.

Logo, a deformação virtual de Green (parte linear) passa a ser dada por:

$$
\delta \varepsilon_{11}^{*}=H^{*} B^{*} \delta q
$$


A taxa da deformação (parte linear) e da deformação virtual de Green, são dadas respectivamente por:

$$
\begin{aligned}
& \dot{\varepsilon}_{11}^{*}=H^{*} B^{*} \dot{q} \\
& \delta \dot{\varepsilon}_{11}^{* \top}=\delta q^{\top}\left(B^{* \top} H^{* \top} H^{*} B^{*}+C^{* \top} C^{*}\right) \dot{q}
\end{aligned}
$$

Utilizando-se a expressão da taxa da equação de equilíbrio, equação (3.3) e desconsiderando-se forças externas atuantes no elemento, tem-se:

$$
\frac{\mathrm{d}}{\mathrm{dt}} \int_{V} \delta \varepsilon^{*^{\top}}\{\sigma\} \mathrm{dV}=\delta \mathrm{q}^{\top} \mathrm{K}_{\mathrm{T}} \dot{\mathrm{q}}
$$

Sabendo-se que:

$$
\frac{d}{d t} \int_{V} \delta \varepsilon^{*^{\top}}\{\sigma\} d V=\int_{V} \delta \varepsilon^{* \top}\left\{\dot{S}^{*}\right\} d V+\int_{V} \delta \dot{\varepsilon}^{\top} \mid \hat{\alpha} d V
$$

e substituindo-se as equações (3.75), (3.87), (3.88) e (3.89) na equação (3.91), tem-se que a expressão da matriz de rigidez tangente para o elemento finito de pórtico plano na configuração atualizada, é dada por:

$$
K_{T}=\int_{V} B^{* \top} H^{* \top} D_{11} H^{*} B^{*} d V+\int_{V} B^{* \top} H^{* \top} \sigma_{11} H^{*} B^{*} d V+\int_{V} C^{* \top} \sigma_{11} C^{*} d V
$$

Realizando-se as integrais da equação (3.92) resultam:

$$
\mathrm{K}_{\mathrm{T}}=\mathrm{K}_{1}+\mathrm{K}_{2}+\mathrm{K}_{3}
$$

onde: 


$$
\int_{V} B^{* \top} H^{* \top} D_{11} H^{*} B^{*} d V=K_{1}
$$

$$
K_{1}=\left[\begin{array}{cccccc}
\frac{E A}{L} & 0 & -\frac{E}{L} \int_{A} y d A & -\frac{E A}{L} & 0 & \frac{E}{L} \int_{A} y d A \\
& \frac{12 E I}{L^{3}} & \frac{6 E I}{L^{2}} & 0 & -\frac{12 E I}{L^{3}} & \frac{6 E l}{L^{2}} \\
& & \frac{4 E I}{L} & \frac{E}{L} \int_{A} y d A & -\frac{6 E I}{L^{2}} & \frac{2 E I}{L} \\
& & \frac{E A}{L} & 0 & -\frac{E}{L} \int_{A} y d A \\
& & & \frac{12 E I}{L^{3}} & -\frac{6 E I}{L^{2}} \\
& & & & \frac{4 E I}{L}
\end{array}\right]
$$

$$
\int_{V} B^{* \top} H^{* \top} \sigma_{11} H^{*} B^{*} d V=K_{2}
$$

$$
K_{2}=\sigma_{11}\left[\begin{array}{cccccc}
\frac{A}{L} & 0 & -\frac{1}{L_{0}} \int_{A} y d A & -\frac{A}{L} & 0 & \frac{1}{L} \int_{A} y d A \\
& \frac{12 l}{L^{3}} & \frac{6 l}{L^{2}} & 0 & -\frac{12 l}{L^{3}} & \frac{6 l}{L^{2}} \\
& \frac{4 I}{L} & \frac{1}{L} \int_{A} y d A & -\frac{6 l}{L^{2}} & \frac{2 l}{L} \\
& \frac{A}{L} & 0 & -\frac{1}{L} \int_{A} y d A \\
\text { simetria } & & & \frac{12 l}{L^{3}} & -\frac{6 l}{L^{2}} \\
& & & & \frac{4 l}{L}
\end{array}\right]
$$




$$
\int_{V} C^{* \top} \sigma_{11} C^{*} d V=K_{3}
$$

$$
\mathrm{K}_{3}=N\left[\begin{array}{cccccc}
0 & 0 & 0 & 0 & 0 & 0 \\
& \frac{6}{5 \mathrm{~L}} & \frac{1}{10} & 0 & -\frac{6}{5 \mathrm{~L}} & \frac{1}{10} \\
& & \frac{2 \mathrm{~L}}{15} & 0 & -\frac{1}{10} & -\frac{\mathrm{L}}{30} \\
& & & 0 & 0 & 0 \\
& & & & \frac{6}{5 \mathrm{~L}} & -\frac{1}{10} \\
\text { simetria } & & & & \frac{2 \mathrm{~L}}{15}
\end{array}\right]
$$

Cabe aqui o mesmo comentário feito na formulação Lagrangiana Total, isto é: $\int_{A} y d A=0$.

Como pode se observar, tanto para elemento de treliça plana quanto para o de pórtico plano, a expressão obtida para a matriz de rigidez tangente da formulação Lagrangiana Atualizada é muito mais simples que a da formulação Lagrangiana Total, no sentido de que uma parcela análoga à matriz dos deslocamentos iniciais não existe (empregou-se a parte linear da deformação virtual).

\subsubsection{ELEMENTO FINITO PARA ANÁLISE BIDIMENSIONAL}

De maneira análoga à formulação Lagrangiana Total, pode-se descrever a formulação Lagrangiana Atualizada. Para isto basta mudar-se da configuração inicial (material) para a configuração atualizada, porém utilizando-se as mesmas interpolações isoparamétricas nas coordenadas atualizadas. Por exemplo, a geometria atualizada é agora aproximada por:

$$
x_{e}=\sum_{i=1}^{n} N_{i} x_{i}
$$


Como as coordenadas globais são dadas com relação à configuração atualizada, as diferenciações devem ser feitas com relação a $x_{a}$, tais como:

$$
\frac{\partial u_{e}}{\partial x_{a}}=\sum_{i=1}^{n} \frac{\partial N_{i}(\xi, \eta)}{\partial x_{a}} q_{i}
$$

Aplicando-se a regra da cadeia, a derivada parcial de $N_{i}$ com relação a $x_{i}$ é dada por:

$$
\left\{\begin{array}{l}
N_{i, 1} \\
N_{i, 2}
\end{array}\right\}=\frac{1}{\operatorname{det} J_{c}}\left[\begin{array}{cc}
x_{2, \eta} & -x_{2, \xi} \\
-x_{1, \eta} & x_{1, \xi}
\end{array}\right]\left[\begin{array}{l}
N_{i, \xi} \\
N_{i, \eta}
\end{array}\right]
$$

$\operatorname{com} J_{c}=\left[\begin{array}{ll}x_{1, \xi} & x_{2, \xi} \\ x_{1, \eta} & x_{2, \eta}\end{array}\right]$

onde det $J_{c}$ é o determinante da transformação Jacobiana entre $d \xi$, $d \eta$ e $d x_{1}$, $\mathrm{dx}_{2}$. Observa-se que as expressões acima são as mesmas da formulação Lagrangiana Total, porém na configuração atualizada.

Neste caso, o tensor de deformação de Green é dado por:

$$
\varepsilon_{\mathrm{e}}^{*}=\frac{1}{2}\left(\operatorname{gradu}+\operatorname{gradu}^{\top}+\operatorname{gradu}^{\top} \operatorname{gradu}\right)
$$

onde gradu é o gradiente dos deslocamentos na última configuração equilibrada.

Portanto, a parte linear da deformação e da deformação virtual de Green podem ser escritas respectivamente por:

$$
\varepsilon_{e}^{*}=\sum_{i=1}^{n} B_{i}^{*} q_{i}
$$


51

$$
\delta \varepsilon_{\mathrm{e}}^{*}=\sum_{i=1}^{\mathrm{n}} \mathrm{B}_{\mathrm{i}}^{*} \delta q_{\mathrm{i}}
$$

onde $\mathrm{B}_{\mathrm{i}}^{*}$ é agora dada por:

$$
B_{i}^{*}=\left[\begin{array}{cc}
N_{i, 1} & 0 \\
0 & N_{i, 2} \\
N_{i, 2} & N_{i, 1}
\end{array}\right]
$$

Levando-se o tensor de deformação de Green na equação de equilibrio, resulta:

$$
r_{e}(q, \delta q)=\sum_{i=1}^{n} \delta q_{i}^{\top}\left\{\int_{\phi \Omega_{e}}\left(B_{i}^{* \top} \sigma_{i}-N_{i} \rho \bar{b}\right) d \phi \Omega_{e}-\int_{\phi \partial \Omega_{e}} N_{i} \bar{t} d \phi \partial \Omega_{e}\right\}=0
$$

Como a equação de equilíbrio não é linear, a sua linearização é dada por:

$$
\frac{d}{d t} r_{e}(q, \delta q) \dot{q}_{i}=\sum_{i=1}^{n} \sum_{k=1}^{n} \delta q_{i}^{\top} \int_{\phi \Omega_{e}}\left\{B_{i}^{* \top} D B_{k}^{*}+G_{i}^{*} G_{k}^{* \top}|\hat{\sigma}|\right\} d \phi \Omega_{e} \dot{q}_{k}=0
$$

onde:

$$
|\hat{a}|=\left[\begin{array}{ll}
\sigma_{11} & \sigma_{12} \\
\sigma_{21} & \sigma_{22}
\end{array}\right] \quad G_{i}^{*}=\left\{\begin{array}{l}
N_{i, 1} \\
N_{i, 2}
\end{array}\right\}
$$

Logo, a matriz de rigidez tangente em coordenadas atualizadas é:

$$
\mathrm{K}_{T_{e}}=\sum_{i=1}^{n} \sum_{k=1}^{n} \int_{\phi \Omega_{e}}\left\{B_{i}^{* \top} D B_{k}^{*}+G_{i}^{*} G_{k}^{* \top}|\hat{\sigma}|\right\} d \phi \Omega_{e}
$$




\section{CAPÍTULO 4- ANÁLISE DA ESTABILIDADE DO EQUILÍBRIO}

\section{1- INTRODUÇÃO À INSTABILIDADE}

$\mathrm{Na}$ análise não-linear geométrica 0 equilíbrio é estabelecido na configuração 'deformada' da estrutura, isto é, levando-se em conta os deslocamentos e as deformações ocorridos. As alterações na geometria da estrutura podem levar à perda de estabilidade do equilíbrio por aparecimento de ponto de bifurcação ou ponto limite.

Ponto de bifurcacão é definido como o ponto a partir do qual a 'trajetória de equilíbrio' definida pelos pares carga-deslocamento que verificam a condição de equilíbrio, deixa de ser única; a este ponto corresponde a chamada carga crítica.

A cada par carga-deslocamento que verifica a condição de equilíbrio está associada uma configuração da estrutura.

Ponto limite é definido como o ponto que encerra uma sucessão de configurações estáveis de equilíbrio, sem aparecimento de bifurcação. Quando a estrutura atinge o ponto limite há uma alteração brusca da sua configuração para uma nova situação estável na trajetória de equilíbrio. 
É importante lembrar que estes conceitos estão ligados à análise da estabilidade estrutural e não à estabilidade de soluções de equações nãolineares, como será mostrado adiante no item 4.5.1.

Os exemplos que seguem ilustram essas definições.

Seja o sistema composto por uma barra rígida com mola, carregada axialmente com uma força $P$, mostrado na figura 4.1(a), na posição deslocada.

Por equilíbrio de momento na extremidade inferior tem-se:

$$
\mathrm{PL} \operatorname{sen} \theta=\mathrm{M} \quad \text { ou } \quad \theta=\frac{\mathrm{PL}}{\mathrm{K}} \operatorname{sen} \theta
$$

uma vez que $M=K \theta$.

Observa-se que a equação de equilíbrio tem 2 soluções, isto é:

$$
\begin{aligned}
& \theta=0 \text { para qualquer valor de } \mathrm{P} \\
& \frac{\mathrm{PL}}{\mathrm{K}}=\frac{\theta}{\operatorname{sen} \theta}
\end{aligned}
$$

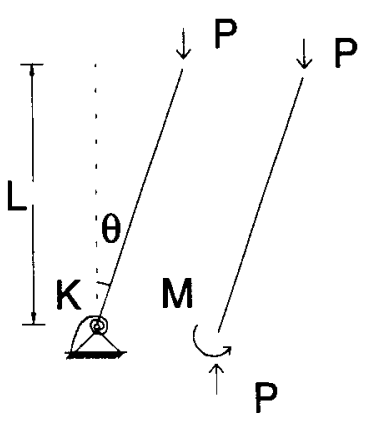

(a)

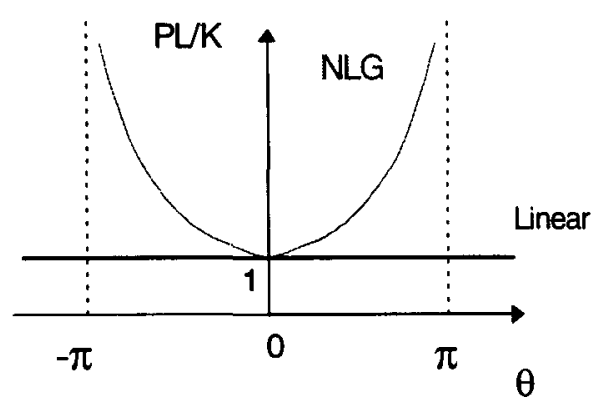

(b)

FIGURA 4.1- Trajetórias de bifurcação de equilíbrio e comportamento pós-flambagemHINTON (1993). 
Assim, apresenta-se outra característica do comportamento nãolinear geométrico: as soluções não são únicas. Na figura 4.1 (b) mostra-se que quando $\mathrm{PL} / \mathrm{K}>1$ a equação de equilíbrio pode ter três soluções para a rotação $\theta$.

A relação (4.2) pode ser escrita na forma $K \theta-P L \operatorname{sen} \theta=0$ portanto, se $\theta \rightarrow 0, \operatorname{sen} \theta \rightarrow \theta$ a equação de equilíbrio fica:

$$
(\mathrm{K}-\mathrm{PL}) \cdot \theta=0
$$

A partir dessa relação 'linearizada' em $\theta$, observa-se que o equilíbrio subsiste se $\theta=0$ para qualquer valor de $P$, e, por outro lado, se $P L / K=1$ a coluna estará em equilíbrio para qualquer valor de $\theta$, como mostra a figura 4.1(b). A interseção das trajetórias de equilíbrio, em $P L / K=1$, é chamada de ponto de bifurcação e o valor, $P=K / L$ é chamado de carga crítica elástica. Diz-se que, a trajetória de equilíbrio resultante da solução $\theta=0$ é estável para $P L / K \leq 1$ e instável para $P L / K>1$, pois no primeiro caso o momento resistente da mola é maior que o solicitante. A trajetória de equilíbrio (NLG), mostrada na figura 4.1(b) é sempre estável e é conhecida como pós-crítica.

Embora simples, este exemplo ilustra as mesmas características apresentadas pelas barras comprimidas. Concluindo, deve-se lembrar que o comportamento não-linear geométrico e a análise de estabilidade linear resultam da consideração do 'deslocamento' $\theta$ causado pela carga $P$ quando se formulam as equações de equilíbrio. As características desse exemplo são próprias de estruturas em que o comportamento não-linear geométrico apresenta grandes deslocamentos / grandes rotações / pequenas deformações.

Seja, por outro lado a estrutura de uma treliça simples representada, em função da geometria, por uma única barra como mostra a figura $4.2(a)$. 


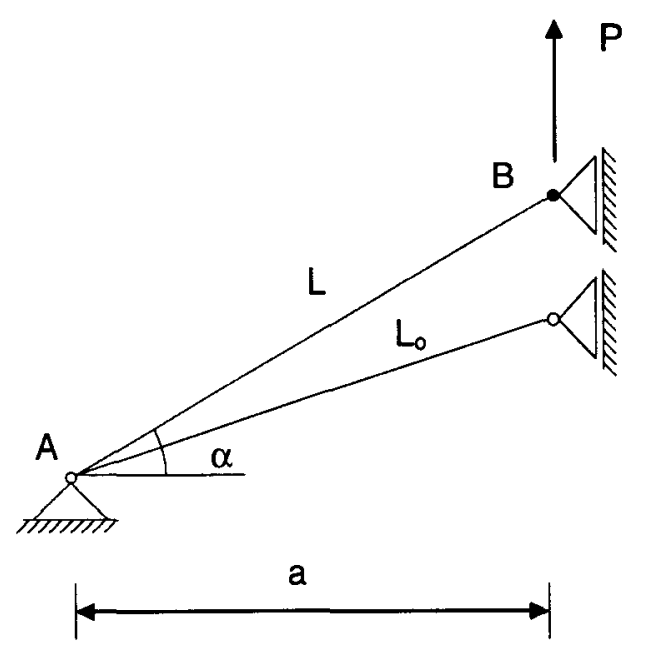

(a)

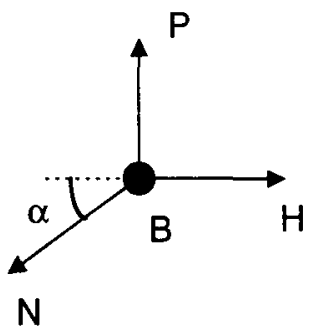

(b)

FIGURA 4.2- Treliça simples

Objetivando-se a aplicação do que segue também para o estudos de cabos considere-se a possibilidade de existir uma força de tração inicial No na barra.

Supondo-se pequenas deformações pode-se admitir que a área $\mathrm{A}$ da seção transversal da barra permanece aproximadamente constante.

De acordo com a figura 4.2(b) o equilíbrio (no ponto B) em termos de onde a estrutura ESTÁ depois do carregamento (descrição Euleriana) é expresso por:

$$
\begin{aligned}
& N \cos \alpha-H=0 \\
& N \frac{(h+u)}{L}-P=0
\end{aligned}
$$

sendo $\mathrm{N}$ a força de tração na barra. 
Introduzindo-se a equação constitutiva e assumindo que o material é elástico linear, tem-se que:

$$
\left(\mathrm{N}-\mathrm{N}_{0}\right)=\mathrm{EA} \varepsilon_{\ell}
$$

$\varepsilon_{\ell}$ é a medida de deformação linear dada por:

$$
\varepsilon_{\ell}=\frac{\left(L-L_{0}\right)}{L_{0}}
$$

onde:

$$
L_{\circ}=a\left[1+\left(\frac{h}{a}\right)^{2}\right]^{\frac{1}{2}} \text { e } L=a\left[1+\left(\frac{h+u}{a}\right)^{2}\right]^{\frac{1}{2}}
$$

Entretanto, neste caso em análise é mais interessante trabalhar com a deformação de Green, definida por:

$$
\varepsilon_{g}=\frac{1}{2}\left(\frac{L^{2}-L_{o}^{2}}{L_{o}^{2}}\right)
$$

e que aplicada ao problema particular em questão resulta:

$$
\varepsilon_{g}=\left(\frac{h}{L_{0}}\right)\left(\frac{u}{L_{0}}\right)+\frac{1}{2}\left(\frac{u}{L_{o}}\right)^{2}
$$

A relação entre a medida de deformação de Green e a medida linear é dada por:

$$
\varepsilon_{\mathrm{g}}=\varepsilon_{\ell}+\frac{\varepsilon_{\ell}^{2}}{2}
$$


Observando-se que $\frac{\varepsilon_{\mathrm{g}}}{\varepsilon_{\ell}}=1+\frac{\varepsilon_{\ell}}{2}$, a equação constitutiva expressa em função da deformação de Green resulta:

$$
N-N_{o}=E A \varepsilon_{g}\left[\frac{1}{2} \cdot\left(\frac{L}{L_{0}}+1\right)\right]^{-1}
$$

Usando as equações (4.9) e (4.11) na equação (4.4b) e assumindose que $L / L_{0} \cong 1$ (para pequenas deformações $\varepsilon_{\ell}<<1$, e portanto a deformação de Green e a deformação linear praticamente se confundem) a equação de equilíbrio fica:

$$
E A\left[\frac{u^{3}+3 h u^{2}+2 h^{2} u}{2 L_{o}^{3}}\right]+N_{o} \frac{(h+u)}{L_{o}}-P=0
$$

Esta equação não é linear com relação ao deslocamento $u$ e é também função da força axial $N_{0}$. Observe que a equação de equilibrio, correspondente à configuração deformada, resultou expressa em termos de grandezas geométricas relativas à posição onde a estrutura ESTAVA antes do carregamento, isto é, uma descrição Lagrangiana (Material) em termos de $A$, $h$ e $L_{o}$.

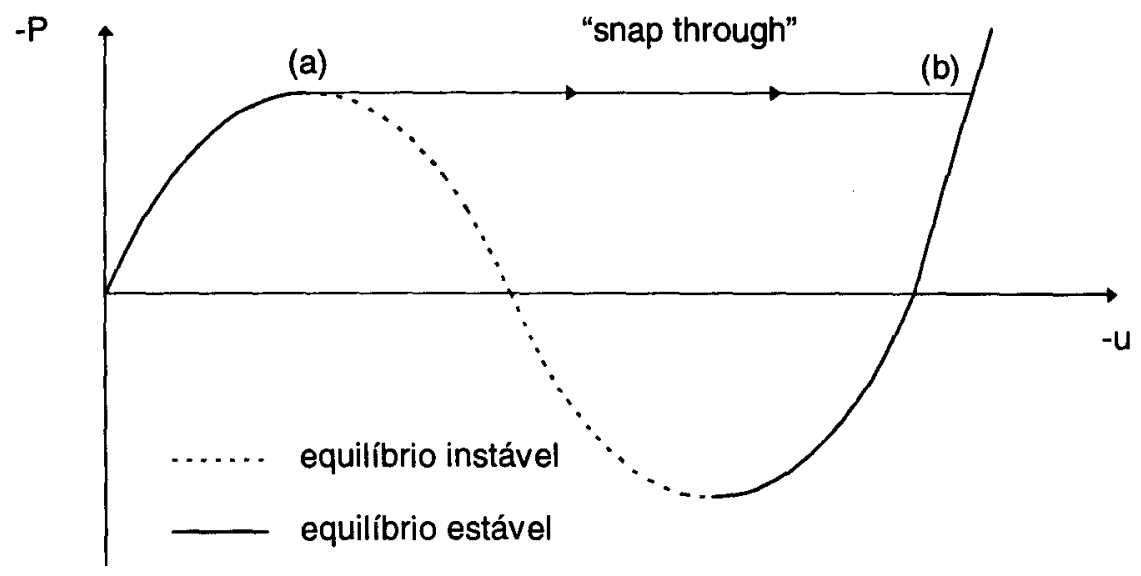

FIGURA 4.3- Comportamento não-linear de uma treliça simples- adaptada de HINTON (1993) 
A trajetória de equilíbrio na figura 4.3, demonstra algumas características importantes, como a chamada carga limite, indicada pelo ponto (a). Na prática esta seria a carga máxima que a estrutura suportaria. Um pequeno incremento na carga acima da carga limite causaria (na prática) um movimento dinâmico violento da treliça seguido pelo restabelecimento do equilíbrio estático no ponto (b).

O movimento (salto) de (a) para (b) é chamado comportamento "snap through" pela literatura inglesa e os resultados deste modelo simples colocam em evidência uma característica do comportamento não-linear geométrico de estruturas com grandes deslocamentos / pequenas rotações / pequenas deformações, como as cascas abatidas, por exemplo.

Apenas com o objetivo de quantificar os resultados obtidos com valores numéricos, considere-se uma treliça de geometria análoga à da figura 4.2(a), porém comprimida e com os seguintes dados:

$$
\begin{array}{ll}
\text { Perfil } \phi 82,5 \times 2,6 \mathrm{~mm} & E=20500 \mathrm{kN} / \mathrm{cm}^{2} \\
L_{0} \cong 150,33 \mathrm{~cm} & \alpha \cong 3,8^{\circ} \\
h=10 \mathrm{~cm} & N_{\circ}=0.0
\end{array}
$$

A figura 4.4 apresenta gráfico carga/deslocamento da equação (4.12), obtido arbitrando-se valores para o deslocamento u e calculando o valor da carga $\mathbf{P}$. 


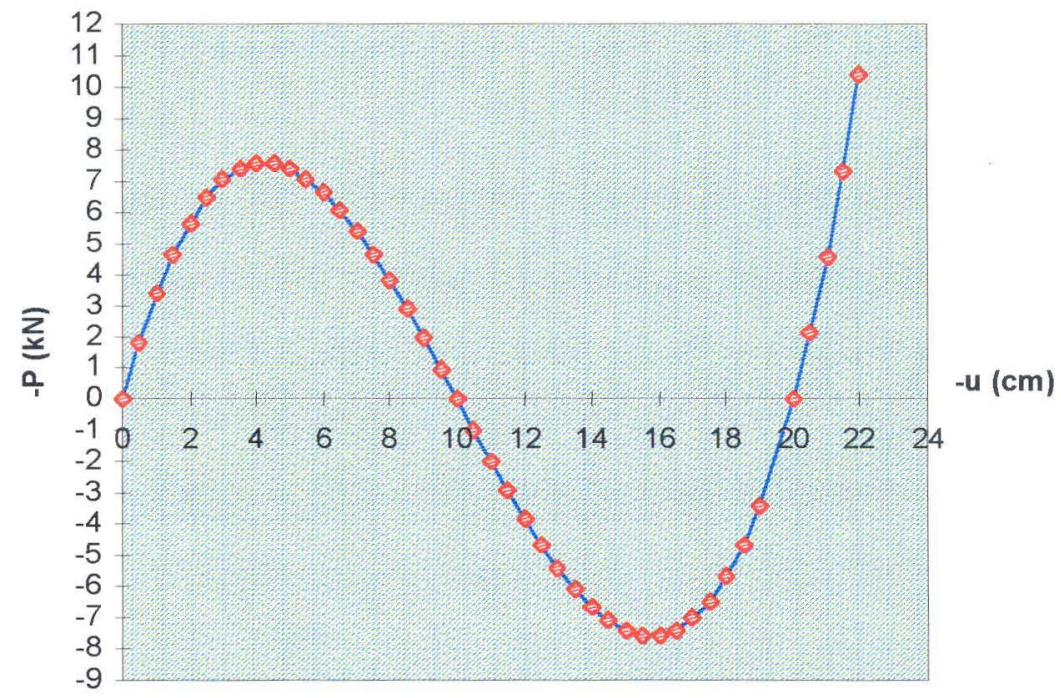

FIGURA 4.4- Curva carga/deslocamento de uma treliça simples

A relação (4.12) pode servir, em particular, para a análise de uma estrutura de cabo. Adotando-se nesse caso, área $A=6,53 \mathrm{~cm}^{2}$, $\mathrm{L}_{\mathrm{o}}=1000 \mathrm{~cm}, \quad \mathrm{~h}=0, \quad \mathrm{~N}_{\mathrm{o}}=100$ e $10000 \mathrm{~N}$, obtêm-se o gráfico carga/deslocamento, ilustrado pela figura 4.5 .

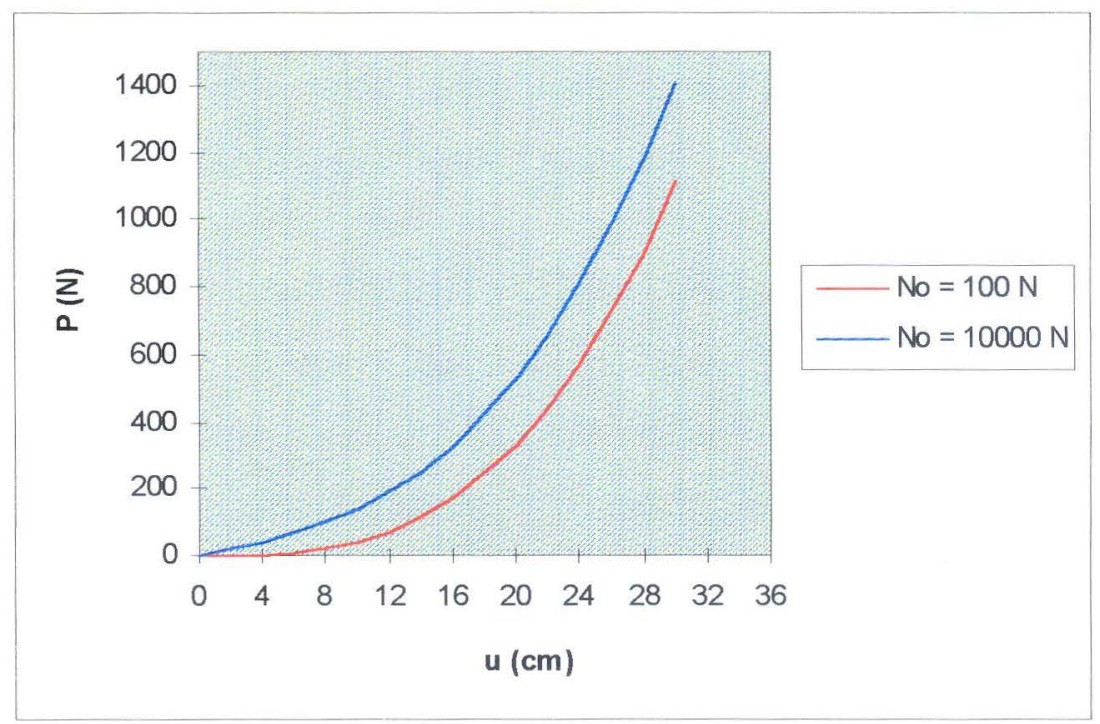

FIGURA 4.5- Curva carga/deslocamento de um cabo 


\section{2- ESTUDO DOS ALGORITMOS PARA CARACTERIZAÇÃO DE PONTOS SINGULARES E DO TIPO DE INSTABILIDADE}

Sabendo-se que na análise não-linear geométrica, a estrutura pode perder a estabilidade do equilíbrio, então, o estudo da resposta não-linear das estruturas deve ser acompanhado de uma análise sobre a estabilidade do 'caminho' de equilíbrio percorrido nas sucessivas etapas de carregamento. Essa análise envolve fundamentalmente a identificação de pontos singulares como os ponto limite e ponto de bifurcação.

Existem vários métodos para determinar singularidades na curva carga/deslocamento, os quais estão baseados na inspeção do determinante da matriz de rigidez tangente (entretanto, esses métodos não permitem identificar o tipo de singularidade). As condições para caracterização da singularidade podem ser inseridas de modo direto na análise através de uma extensão do sistema de equações não-lineares, adicionando-se uma condição de restrição que introduz informação adicional sobre os pontos de perda de estabilidade (pontos singulares). Estes sistemas consistentemente linearizados, preservam a convergência quadrática, típica do Método de Newton.

Os métodos numéricos para o cálculo de pontos singulares podem ser classificados em duas categorias: direto $e$ indireto. $O$ método direto baseia-se nos sistemas estendidos enquanto que o indireto em interpolação.

De acordo com WEINITSCHKE (1985), para calcular com exatidão pontos singulares na curva carga/deslocamento utilizando-se o método de interpolação é necessário um grande número de pontos, o que se torna difícil por causa da singularidade próxima dos mesmos. Por isto, os métodos indiretos de cálculo de pontos singulares são insatisfatórios e de pouca exatidão, principalmente em caso de pontos limite. 
Restringe-se neste trabalho ao estudo dos sistemas estendidos, visto que estes abrem possibilidade para se computar os pontos limite ou de bifurcação diretamente.

\section{3- MÉTODO INCREMENTAL ITERATIVO DE NEWTON- RAPHSON}

O método de Newton-Raphson é muito eficiente para solução de sistemas não-lineares como, por exemplo, o sistema de equações dado pela equação (3.8) que pode ser escrito na forma:

$$
G(q)=R(q)-P=0
$$

onde:

$$
\begin{aligned}
& G(q)=\int_{V_{0}} r_{0} \cdot \delta u d V_{0}, R(q)=\int_{V_{0}} S \cdot \delta \varepsilon d V_{0} \\
& P=\int_{V_{0}} b_{0} . \delta u d V_{0}+\int_{A_{0}} \bar{t}_{0} . \delta u d A_{0}
\end{aligned}
$$

Segundo a estratégia de solução, o carregamento total é aplicado em vários incrementos e nesse sentido é interessante introduzir na equação (4.13) um escalar $\lambda$, chamado parâmetro de carga. Assim, o sistema nãolinear passa a ser representado na forma:

$$
G(q, \lambda)=R(q)-\lambda P=0
$$

O método incremental iterativo de Newton-Raphson consiste, essencialmente, em tentar anular o resíduo $\mathrm{G}(\mathrm{q})$ gerado pela forma linearizada da equação (4.14) em cada passo de carga. Assim, a solução terá sido encontrada quando $G(q, \lambda) \cong 0$. 
O desenvolvimento do resíduo $\mathrm{G}$ em torno de uma vizinhança, é dado por:

$$
G^{t+\Delta t}(q, \lambda)=G^{t}(q, \lambda)+\frac{d}{d t} G^{t}(q, \lambda) \Delta q+O(\Delta q)
$$

Admitindo-se que no instante $t+\Delta t, G^{t+\Delta t}=0$, tem-se que:

$$
\frac{d}{d t} G^{t}(q, \lambda) \Delta q=-G^{t}(q, \lambda)
$$

A equação (4.15) permite determinar o vetor incremento dos deslocamentos nodais $\Delta \mathrm{q}$.

Por outro lado, considerando-se apenas forças conservativas, temse que:

$$
\frac{d}{d t} G(q, \lambda) \Delta q=K_{T} \Delta q
$$

Deste modo a equação (4.15) passa a ser dada por:

$$
K_{T}(q) \Delta q=-G(q, \lambda)
$$

Considere-se que o carregamento seja incrementado do valor $P_{i}$ para $\mathrm{P}_{\mathrm{i}+1}$ aplicando-se um incremento $\lambda_{\mathrm{i}}$. Deste modo, um primeiro valor aproximado é obtido de:

$$
\mathrm{K}_{\mathrm{Ti}} \Delta \mathrm{q}_{\mathrm{i}}^{0}=\lambda_{\mathrm{i}} \text { onde } \lambda_{\mathrm{i}}=\mathrm{P}_{\mathrm{i}+1}-\mathrm{P}_{\mathrm{i}}
$$

Portanto, a primeira estimativa para 0 vetor deslocamento correspondente ao valor de carga $\mathrm{P}_{\mathrm{i}+1}$ é obtido como:

$$
q_{i+1}^{1}=q_{i}+\Delta q_{i}^{0}
$$


Com este novo valor para o vetor deslocamento, verifica se o critério de convergência é atendido, se não resolve-se novamente o sistema com rigidez atualizada, encontrando-se um novo incremento para os deslocamentos:

$$
\mathrm{K}_{\mathrm{Ti+1}}^{1} \Delta \mathrm{q}_{\mathrm{i}}^{1}=\mathrm{P}_{\mathrm{i}+1}-\mathrm{F}_{\mathrm{i}+1}^{1}
$$

e portanto,

$$
q_{i+1}^{2}=q_{i+1}^{1}+\Delta q_{i}^{1}
$$

Com este valor para o deslocamento verifica se 0 critério de convergência é atendido, repetindo-se o processo até que este seja satisfeito.

A figura seguinte ilustra o procedimento descrito.

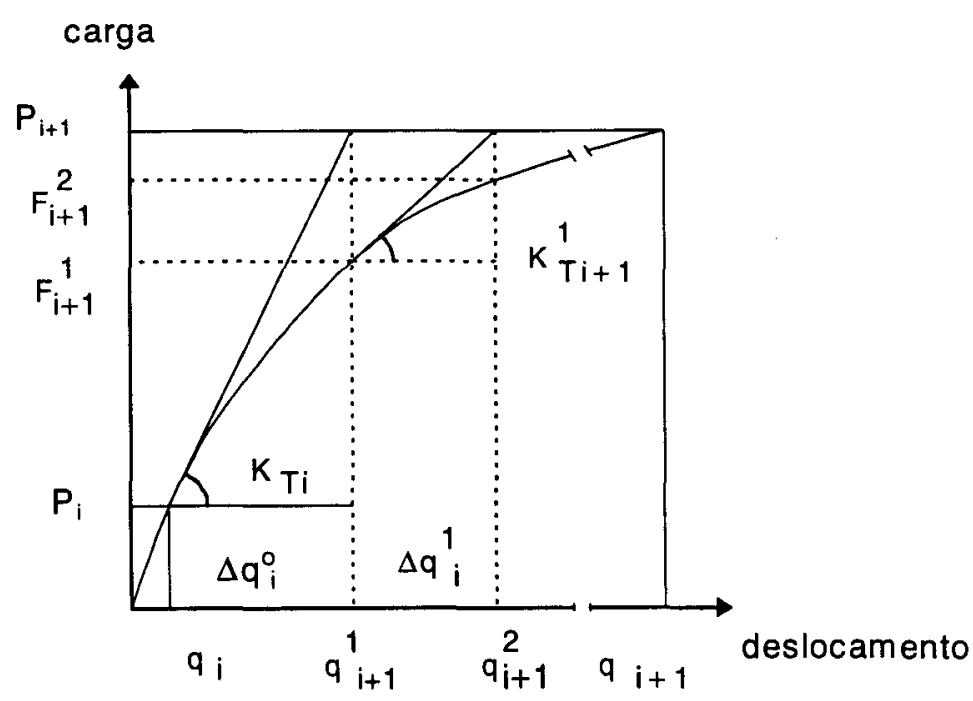

FIGURA 4.6- Método de Newton-Raphson - adaptado de KANCHI (1993) 


\section{4- MÉTODOS DE CONTINUAÇÃO}

De modo análogo ao procedimento de Newton, o sistema de equações algébricas dado pela equação (3.8), pode ser reescrito introduzindo-se um fator escalar $\lambda$, chamado parâmetro de carga, do seguinte modo:

$$
G(q, \lambda)=R(q)-\lambda P=0 \quad q \in R^{N}
$$

onde:

$$
R(q)=\int_{V_{0}} \delta \varepsilon^{\top}\{S\} d V_{0} \text { e } P=\int_{V_{0}} b_{0}^{\top} \delta u d V_{0}+\int_{A_{0}} \bar{T}_{0}^{\top} \delta u d A_{0}
$$

$\mathrm{Na}$ hipótese de ocorrerem pontos singulares, os métodos tipo Newton-Raphson falham, pois particularmente no caso de pontos limites não é possível prosseguir na análise. Nestes casos, os métodos do tipo comprimento de arco ou de continuação são indicados. A principal idéia dos métodos de continuação é introduzir $\lambda$ como incógnita, adicionando-se uma equação de restrição ao sistema de equações não-lineares (equação (4.17)). Assim a forma geral do sistema estendido passa a ser dada por:

$$
\bar{G}(q, \lambda)=\left\{\begin{array}{l}
G(q, \lambda) \\
f(q, \lambda)
\end{array}\right\}=0
$$

onde $f(q, \lambda)=0$ é a equação de restrição. Baseando-se nesta formulação, métodos tipo controle de carga, controle de deslocamento ou comprimento de arco podem ser deduzidos. Como o sistema estendido não é linear, é conveniente trabalhar com sua forma linearizada em torno do ponto i:

$$
\left(\begin{array}{cc}
\nabla_{q} G & \nabla_{\lambda} G \\
\left(\nabla_{q} f\right)^{\top} & \nabla_{\lambda} f
\end{array}\right\}_{i}\left\{\begin{array}{l}
\Delta q \\
\Delta \lambda
\end{array}\right\}_{i}=-\left\{\begin{array}{l}
G \\
f
\end{array}\right\}_{i}
$$


$\mathrm{Na}$ literatura, $\nabla_{\mathrm{q}} \mathrm{G}$ é conhecido como matriz de rigidez tangente enquanto que $P=-\nabla_{\lambda} G$.

O sistema de equações dado pela equação (4.19), não-simétrico, pode ser resolvido pelo método da partição, como mostrado em seguida. $\mathrm{Da}$ primeira equação de (4.19) tem-se que:

$$
\begin{aligned}
& \mathrm{K}_{\mathrm{Ti}} \Delta \mathrm{q}_{\mathrm{i}}-\mathrm{P}_{\mathrm{i}} \Delta \lambda_{\mathrm{i}}=-\mathrm{G}_{\mathrm{i}} \\
& \Delta \mathrm{q}_{\mathrm{i}}=\mathrm{K}_{\mathrm{T}}^{-1} \mathrm{P}_{\mathrm{i}} \Delta \lambda_{\mathrm{i}}-\mathrm{K}_{\mathrm{T}}^{-1} \mathrm{G}_{\mathrm{i}}
\end{aligned}
$$

denominando-se:

$$
\Delta q_{P i}=\left(K_{T i}\right)^{-1} P_{i} \quad \text { e } \quad \Delta q_{G i}=-\left(K_{T i}\right)^{-1} G_{i}
$$

resulta uma expressão para o incremento total do deslocamento, na forma:

$$
\Delta q_{i}=\Delta \lambda_{i} \Delta q_{P i}+\Delta q_{G i}
$$

A partir daí, o incremento do fator de carga (incógnito) é calculado usando-se a segunda equação de (4.19), resultando:

$$
\Delta \lambda_{i}=-\frac{f_{i}+\left(\nabla_{q} f_{i}\right)^{\top} \Delta q_{G i}}{\nabla_{\lambda} f_{i}+\left(\nabla_{q} f_{i}\right)^{\top} \Delta q_{p i}}
$$

$\mathrm{Na}$ literatura, os vários métodos de continuação diferem apenas na equação de restrição; algumas delas estão mostradas na tabela 4.1. 
TABELA 4.1- Exemplos de equações de restrição - WRIGGERS (1990)

\begin{tabular}{|c|c|}
\hline Método & Equação de restrição \\
\hline Controle de carga & $f=\lambda-c$ \\
\hline $\begin{array}{c}\text { Controle de deslocamento } \\
\text { Batoz, Dhatt (1979) }\end{array}$ & $f=q_{a}-c$ \\
\hline $\begin{array}{c}\text { Método comprimento de arco } \\
\text { Riks(1972) }\end{array}$ & $f=\left(q_{m}-\bar{q}\right)^{\top}\left(q-q_{m}\right)+\left(\lambda_{m}-\bar{\lambda}\right)\left(\lambda-\lambda_{m}\right)$ \\
\hline $\begin{array}{c}\text { Método comprimento de arco } \\
\text { Crisfield(1981) }\end{array}$ & $f=\sqrt{(q-\bar{q})^{\top}(q-\bar{q})+(\lambda-\bar{\lambda})^{2}}-d s$ \\
\hline
\end{tabular}

A constante c, da tabela acima, é o parâmetro de referência que limita o vetor deslocamento e o fator de carga dentro do método de continuação; os outros parâmetros são ilustrados pelas figuras 4.7a e 4.7b.

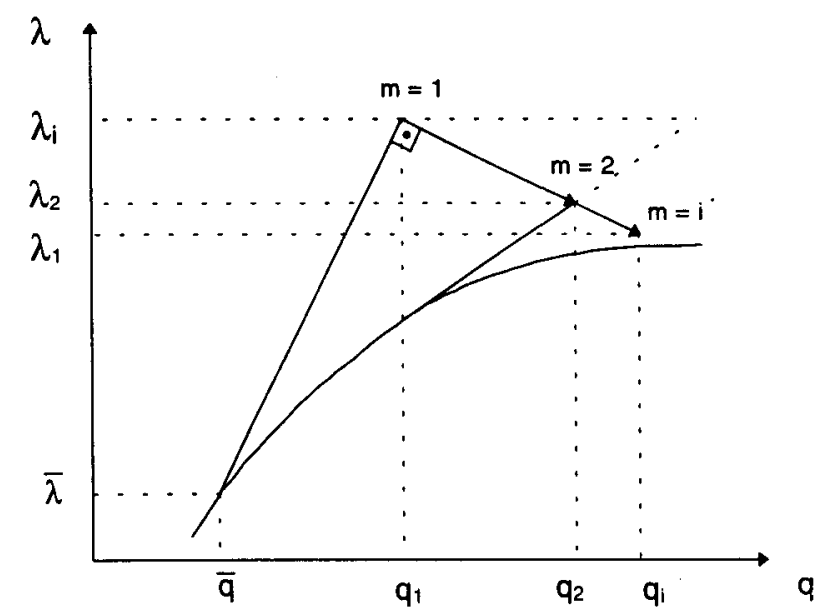

FIGURA 4.7a- Método de comprimento de arco com iteração restrita em um 'plano normal' WRIGGERS (1990) 


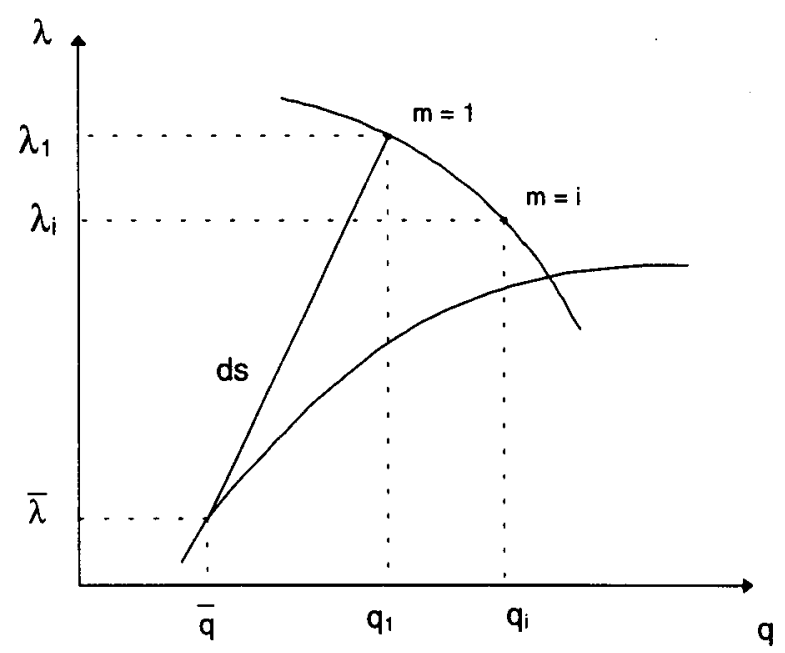

FIGURA 4.7b- Método de comprimento de arco com iteração restrita a uma circunferência de raio ds - WRIGGERS (1990)

Um algoritmo associado aos métodos de continuação é dado pelo quadro 4.1 que segue.

1- Cálculo inicial $\quad \mathrm{K}_{\mathrm{T}} \Delta \mathrm{q}_{\mathrm{Po}}=\mathrm{P}$

2- Cálculo do incremento inicial de carga $\Delta \lambda_{0}=\frac{\mathrm{ds}}{\sqrt{\left(\Delta \mathrm{q}_{\mathrm{P}_{\mathrm{o}}}\right)^{\top} \Delta \mathrm{q}_{\mathrm{Po}_{\mathrm{o}}}}}$

3- "Loop" de todas as iterações i

$\mathrm{K}_{\mathrm{T}} \Delta \mathrm{q}_{\mathrm{Pi}}=\mathrm{P}_{\mathrm{i}}$

$\mathrm{K}_{\mathrm{T}} \Delta \mathrm{q}_{\mathrm{Gi}}=-\mathrm{G}\left(\mathrm{q}_{\mathrm{i}}, \lambda_{\mathrm{i}}\right)$

4- Cálculo dos incrementos

$\Delta \lambda_{i}=-\frac{f_{i}+\left(\nabla_{q} f_{i}\right)^{\top} \Delta q_{G_{i}}}{\nabla_{\lambda} f_{i}+\left(\nabla_{q_{i}}\right)^{\top} \Delta q_{p i}}$

$\Delta q_{i}=\Delta \lambda_{i} \Delta q_{P i}+\Delta q_{G i}$

5- Atualização

$\lambda_{i+1}=\lambda_{i}+\Delta \lambda_{i}, q_{i+1}=q_{i}+\Delta q_{i}$

6- Critério de convergência se $\| G\left(q_{i+1}, \lambda_{i+1} \| \leq T O L \quad\right.$ terminar se não voltar ao passo 3 


\section{5- PONTOS DE SINGULARIDADE}

O algoritmo para solução da equação (4.19) pode ser utilizado para a determinação da trajetória de equilíbrio. Porém esse mesmo algoritmo não é capaz de caracterizar singularidades associadas a pontos limite ou de bifurcação, definidos anteriormente. Assim, outros procedimentos são necessários para a identificação de pontos singulares na trajetória não-linear carga/deslocamento. Tais procedimentos são baseados em sistemas estendidos que incorporam uma outra equação de restrição ao sistema de equações (4.17). A partir da linearização do sistema resultante, algoritmos do tipo Newton podem ser construídos e, como resultado, obtêm-se o parâmetro de carga, o campo dos deslocamentos e o autovetor associado ao ponto singular.

Um objetivo final deste estudo nesse campo de análise, consistiria em identificar a classe dos algoritmos, para implementação em combinação com o método dos elementos finitos, que seja mais eficaz na caracterização de pontos limite ou de bifurcação.

\subsection{1- DEFINIÇÃO DO CRITÉRIO MATEMÁtICO PARA CARACTERIZAÇÃo DE PONTOS LIMITE E DE BIFURCAÇÃO}

Uma vez que tenha sido identificado um ponto singular na curva solução, existe a necessidade de sua caracterização, ou seja, dizer se o ponto é ponto limite ou de bifurcação. A identificação de pontos singulares numa análise numérica é feita utilizando-se 0 critério matemático apresentado a seguir.

Segundo DECKER \& KELLER (1980), pontos de bifurcação de equações não-lineares do tipo $G(u, \lambda)=0$, são pontos $\left(u_{0}, \lambda_{0}\right)$ nos quais dois 
ramos distintos de soluções interceptam-se não-tangencialmente. Já pontos limite, são pontos $\left(u_{0}, \lambda_{0}\right)$ nos quais a derivada de Frechet $\left(G_{u}^{o} \equiv G_{u}\left(u_{0}, \lambda_{0}\right)\right)$ é singular e, além disso, $G_{\lambda}^{\circ} \equiv G_{\lambda}\left(u_{0}, \lambda_{0}\right)$ não está no conjunto imagem de $G_{u}^{0}$.

Para uma melhor interpretação dessas definições matemáticas, é importante neste ponto lembrar a forma (4.17) e observar que $G_{u}$ representa, no problema estrutural, a rigidez tangente e $G_{\lambda} \circ$ vetor de cargas aplicadas.

As figuras $4.8(a)$ e $4.8(b)$ mostram os dois tipos mais comuns de pontos singulares de acordo com as definições acima.

A figura 4.8(a) mostra uma solução cujo ponto singular, ponto $P$, é um ponto limite.

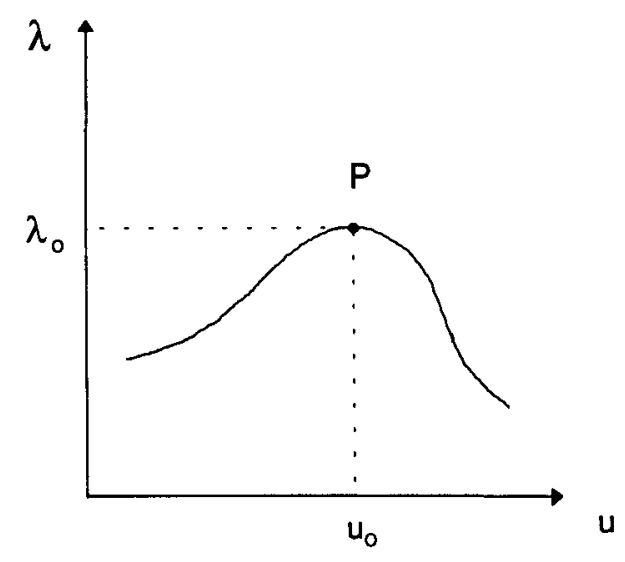

FIGURA 4.8(a)- Ponto limite

A figura 4.8(b) apresenta a bifurcação em $P$ numa situação em que para o ramo 1, o ponto é limite. Este caso é particularmente chamado de bifurcação em ponto de dobra. 


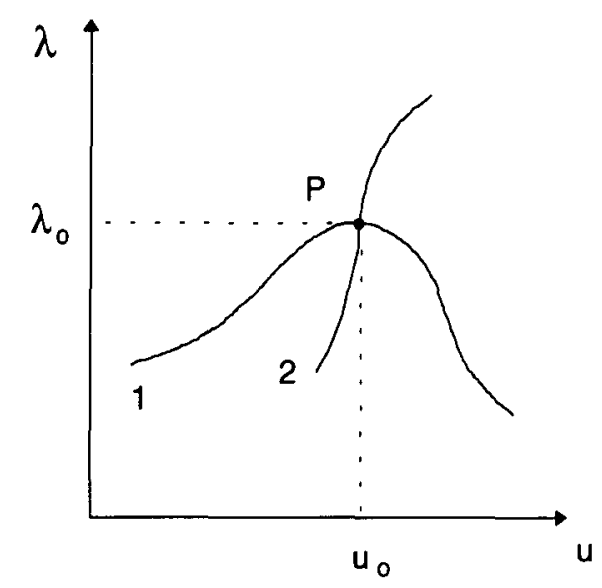

FIGURA 4.8(b)- Bifurcação em ponto de dobra

A figura 4.8(c) ilustra um outro caso particular, que não será tratado aqui, denominado bifurcação em ponto limite; note-se que no ponto limite diferentes curvas solução interceptam-se com a mesma tangente.

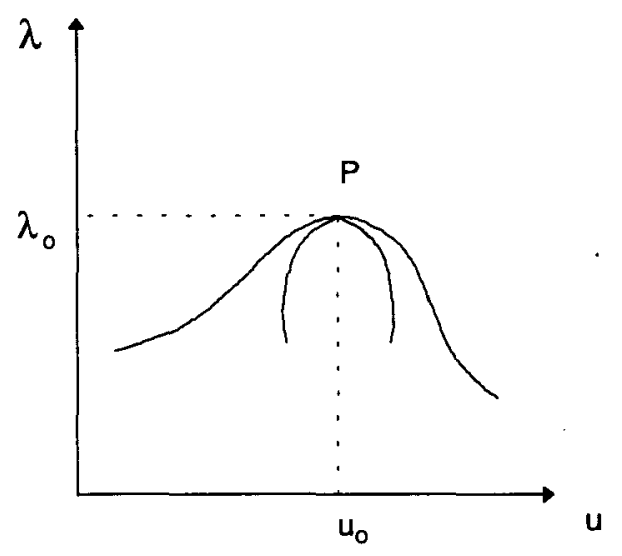

FIGURA 4.8(c)- Bifurcação em ponto limite

Imaginando-se que as curvas-solução $(u(\varepsilon), \lambda(\varepsilon))$ sejam parametrizadas por $\varepsilon$, a principal distinção nestas figuras é que $d \lambda(\varepsilon) / d \varepsilon=0$ num ponto limite, mas não em bifurcação em ponto de dobra. Além disso, admite-se que pontos limite não podem ocorrer num ramo de solução trivial 
$[u, \lambda] \equiv[0, \lambda]$; esse tipo de solução é usualmente considerado em problemas de bifurcação, como o de colunas sob compressão centrada.

Retomando-se a relação:

$$
G(u, \lambda)=0
$$

matematicamente tem-se que $u$ é um elemento de um espaço de Banach $\mathrm{X}$, $\lambda$ é um parâmetro real e $G$ é um operador que leva $X \times R$ a elementos de um espaço de Banach $Y$. Para melhor entendimento ver estudo apresentado em anexo.

Admitindo-se que um ponto $\left(u_{0}, \lambda_{0}\right)$ é conhecido, a condição necessária para sua caracterização como ponto limite ou de bifurcação é que a derivada de Frechet de $G$ com relação a $u$, indicada por $G_{u}^{o} \equiv G_{u}\left(u_{o}, \lambda_{o}\right)$, seja singular (em caso contrário o teorema da função implícita garante existência e unicidade de solução, ver DECKER \& KELLER (1980)).

No que segue, admite-se que a imagem de $G_{u}^{0}$ é fechada, de dimensão finita $m$ e que o núcleo $N\left(G_{u}^{\circ}\right)$ é também um subespaço de dimensão $m$, gerado por um conjunto de versores $\phi_{i}$ linearmente independentes, ou seja:

$$
N\left(G_{u}^{o}\right)=\operatorname{span}\left\{\phi_{1}, \ldots, \phi_{m}\right\}\left\|\phi_{i}\right\|=1 \quad i=1, \ldots, m
$$

Para o operador adjunto ou dual $G_{u}^{0^{*}:} Y^{*} \longrightarrow X^{*}$, admite-se também a existência de um núcleo gerado por um conjunto de $\mathrm{m}$ versores linearmente independentes:

$$
N\left(G_{u}^{0^{*}}\right)=\operatorname{span}\left\{\psi_{1}^{*}, \ldots, \psi_{m}^{*}\right\}
$$


Tendo-se em vista que $R\left(G_{u}^{0}\right)=N\left(G_{u}^{\circ *}\right)^{\perp}$, então a imagem de $G_{u}^{0}$ é caracterizada por:

$$
R\left(G_{u}^{0}\right)=\left\{y \in Y / \psi_{i}^{*} y=0 ; i=1, \ldots, m\right\}
$$

Observa-se também que no espaço dual $\mathrm{X}^{\star}$ existe um conjunto de vetores linearmente independentes $\phi_{i}^{*}(\operatorname{com} i=1, \ldots, \mathrm{m})$ satisfazendo:

$$
\phi_{i}^{*} \phi_{j}=\delta_{i j} \quad i, j=1, \ldots, m
$$

Isso posto, considere-se o estudo de soluções $(u(\varepsilon), \lambda(\varepsilon))$ de $(4.23)$ que passam por $\left(u_{o}, \lambda_{0}\right)$ e que dependam regularmente de um parâmetro $\varepsilon$. A partir disto, derivam-se relações que devem necessariamente ser satisfeitas em $\left(u_{0}, \lambda_{0}\right)$.

Sejam então pares $(u, \lambda)$ parametrizados por $\varepsilon$ tais que:

$$
G(u(\varepsilon), \lambda(\varepsilon))=0 \quad|\varepsilon| \leq\left\|\varepsilon_{0}\right\|
$$

com $u(0), \lambda(0)$ definindo uma trajetória inicial de equilíbrio. Então $\mathrm{G}(u(\varepsilon), \lambda(\varepsilon))=0$ e $\mathrm{G}(u(\varepsilon+\Delta \varepsilon), \lambda(\varepsilon+\Delta \varepsilon))=0$. Portanto:

$\mathrm{G}(\mathrm{u}(\varepsilon+\Delta \varepsilon), \lambda(\varepsilon+\Delta \varepsilon))=$

$$
=G(u(\varepsilon), \lambda(\varepsilon))+\left[G_{\varepsilon}(u(\varepsilon), \lambda(\varepsilon))\right] \Delta \varepsilon+\frac{1}{2}\left[G_{\varepsilon \varepsilon}(u(\varepsilon), \lambda(\varepsilon))\right] \Delta \varepsilon^{2}+\ldots=0
$$

Particularizando-se a última relação para um desenvolvimento em segunda ordem em torno de $\varepsilon=0$ no ponto $\left(u_{0}, \lambda_{0}\right)$ e tendo-se em vista a generalidade dada a $\Delta \varepsilon$, tem-se que $G_{\varepsilon}^{\circ}=0$ e $G_{\varepsilon \varepsilon}^{o}=0$. Sendo $G_{\varepsilon}^{\circ}=\left.\left(G_{u}\left(u_{o}, \lambda_{o}\right) u_{\varepsilon}+G_{\lambda}\left(u_{o}, \lambda_{o}\right) \lambda_{\varepsilon}\right)\right|_{\varepsilon=0}$ resultam: 


$$
\begin{gathered}
G_{u}^{o} u_{\varepsilon}+G_{\lambda}^{o} \lambda_{\varepsilon}=G_{u}^{o} \dot{u}(0)+G_{\lambda}^{o} \dot{\lambda}(0)=0 \\
G_{u}^{o} \ddot{u}(0)+G_{\lambda}^{\circ} \ddot{\lambda}(0)=-\left(G_{u u}^{o} \dot{u}(0)^{2}+2 G_{u \lambda}^{o} \dot{u}(0) \dot{\lambda}(0)+G_{\lambda \lambda}^{o} \dot{\lambda}(0)^{2}\right)
\end{gathered}
$$

onde $G_{u u}^{o}$ é a segunda derivada de Frechet com relação a $u$, calculada em $\left(u_{0}, \lambda_{o}\right), \dot{u}(0)=\left.(d u / d \varepsilon)\right|_{\varepsilon=0}$, etc.

A hipótese de mais de uma solução em $\left(u_{0}, \lambda_{0}\right)$ implica em admitir a existência de $\dot{u}(0)$. Nessas condições, a equação (4.29) é obedecida satisfazendo-se a uma das duas possibilidades distintas seguintes:

$$
G_{\lambda}^{o} \in R\left(G_{u}^{o}\right)
$$

lembrando-se que $\dot{\lambda}$ é um escalar, ou

$$
G_{\lambda}^{o} \notin R\left(G_{u}^{o}\right) \operatorname{mas} \dot{\lambda}(0)=0
$$

A primeira possibilidade tem correspondência com uma bifurcação regular de solução e a segunda com um ponto limite.

Para o caso dado por (4.31i), é possível encontrar um $\phi_{0}$ tal que:

$$
\mathrm{G}_{u}^{\circ} \phi_{\mathrm{o}}=-\mathrm{G}_{\lambda}^{\circ}
$$

onde o sinal negativo aparece por conveniência algébrica.

Substituindo-se (4.32) em (4.29) resulta $G_{u}^{\circ}\left[\dot{u}(0)-\phi_{0} \dot{\lambda}(0)\right]=0$. Como o termo entre colchetes pertence à nulidade de $G_{u}^{0}$ ele pode ser escrito como uma combinação linear dos versores $\phi_{i}(i=1, \ldots, m)$, ou seja, $\dot{u}(0)-\phi_{0} \dot{\lambda}(0)=\xi_{1} \phi_{1}+\xi_{2} \phi_{2}+\ldots+\xi_{m} \phi_{m}$. 
De outro modo, a solução geral pode ser dada por:

$$
\dot{u}(0)=\sum_{j=0}^{m} \xi_{j} \phi_{j} \quad \text { com } \quad \xi_{o}=\dot{\lambda}(0)
$$

Observa-se ainda que, fazendo-se uso da equação (4.26), $\mathrm{G}_{\lambda}^{0} \psi_{\mathrm{i}}^{*}=0 \quad(\mathrm{i}=1, \ldots, \mathrm{m})$ ou, com a equação (4.32), $\mathrm{G}_{u}^{0} \phi_{0} \psi_{i}^{*}=0$ a qual é verificada para um $\phi_{0}$ genérico não nulo se $G_{u}^{0} \psi_{i}^{*}=0$. Portanto, $\psi_{i}^{*}$ é um autovetor associado a $G_{u}^{0}$.

Assim, da análise anterior resulta o seguinte critério para caracterização de ponto de bifurcação na trajetória de solução do sistema de equações não-lineares:

$$
\psi_{i}^{*} G_{\lambda}^{o}=0
$$

sendo que nessas condições, $G_{\lambda}^{0} \in R\left(G_{u}^{0}\right)$ e $\dot{u}(0) \neq 0$.

O critério de bifurcação resultou de uma análise da relação (4.29). Porém, a relação (4.30) permite estabelecer um indicativo sobre o tipo de bifurcação, a qual pode ser simples, em garfo ou em 'ilha', como mostra a figura 4.9. 


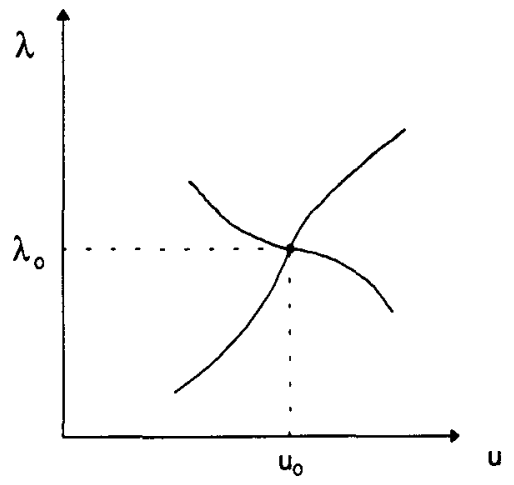

Bifurcação simples

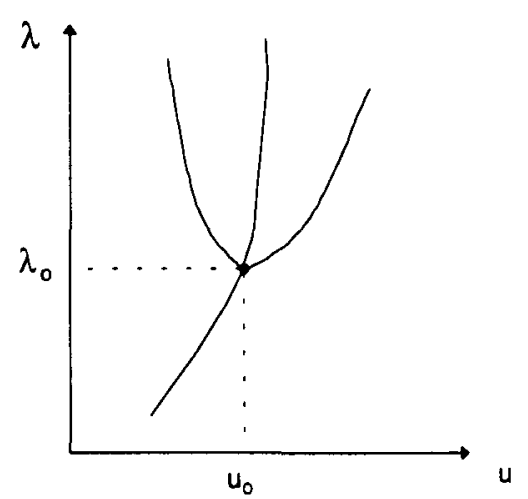

Bifurcação em garfo

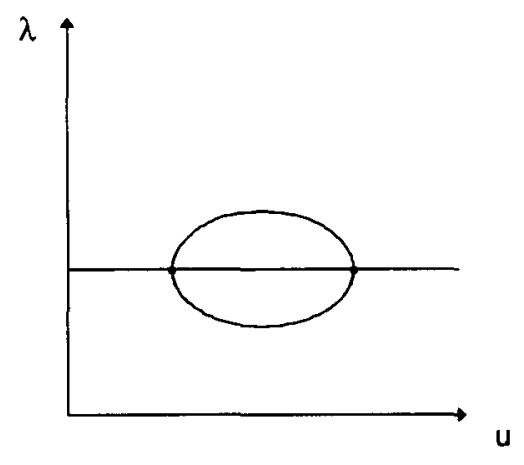

Bifurcação em 'ilha'

FIGURA 4.9- Tipos de bifurcação

Substituindo-se na equação (4.30) a solução dada por (4.33), escrita na forma $\dot{u}(0)=\sum_{j=1}^{m} \xi_{j} \phi_{j}+\xi_{o} \phi_{o}$, obtém-se:

$$
\begin{aligned}
& G_{u}^{o} \ddot{u}(0)+G_{\lambda}^{o} \ddot{\lambda}(0)+G_{u u}^{o} \sum_{j=1}^{m} \sum_{k=1}^{m} \xi_{j} \xi_{k} \phi_{j} \phi_{k}+2 G_{u u}^{o} \sum_{j=1}^{m} \xi_{j} \phi_{j} \xi_{o} \phi_{o}+G_{u u}^{o} \xi_{o}^{2} \phi_{o}^{2}+ \\
& +2 G_{u \lambda}^{o} \sum_{j=1}^{m} \xi_{j} \phi_{j} \xi_{o}+2 G_{u \lambda}^{o} \xi_{o}^{2} \phi_{o}+G_{\lambda \lambda}^{o} \xi_{o}^{2}=0
\end{aligned}
$$


Multiplicando-se a equação (4.35) por $\psi_{i}^{*}$, considerando-se (4.31i) e (4.26), encontra-se uma condição necessária para a existência de uma 'solução' $\ddot{u}(0)$ :

$$
\sum_{k=1}^{m} \sum_{j=1}^{m} a_{i j} \xi_{j} \xi_{k}+2 \sum_{j=1}^{m} b_{i j} \xi_{j} \xi_{o}+c_{i} \xi_{o}^{2}=0 \quad i=1, \ldots, m
$$

onde:

$$
\begin{aligned}
& a_{i \mathrm{ik}} \equiv \psi_{i}^{*} G_{u u}^{o} \phi_{j} \phi_{k} \\
& b_{i j} \equiv \psi_{i}^{*}\left(G_{u u}^{o} \phi_{o}+G_{u \lambda}^{o}\right) \phi_{j} \\
& c_{i} \equiv \psi_{i}^{*}\left(G_{u u}^{o} \phi_{o}^{2}+2 G_{u \lambda}^{o} \phi_{o}+G_{\lambda \lambda}^{\circ}\right) \\
& \phi_{o}=\text { solução de equação (4.32). }
\end{aligned}
$$

A equação (4.36) é chamada de equação de bifurcação na literatura.

Define-se também $\Delta=b_{i j}^{2}-a_{i j k} c_{i}$ e a partir daí transcreve-se sem maiores demonstrações, a classificação proposta por JEPSON \& SPENCE (1985):

- se $a_{\mathrm{ijk}} \neq 0$ e $\Delta>0$ o ponto é de bifurcação simples.

- se $a_{i j k}=0$ e $b_{i j} \neq 0$ o ponto é de bifurcação em garfo.

- se $\Delta<0$ o ponto é de bifurcação em 'ilha'.

Por outro lado, para a possibilidade (4.31ii) não existe um vetor $\phi_{0}$ que satisfaça (4.32) e assim, a solução geral de (4.29) deve ser substituída por: 


$$
\dot{u}(0)=\sum_{j=1}^{m} \xi_{j} \phi_{j} \quad \xi_{o}=\dot{\lambda}(0)=0
$$

Neste caso, trata-se de um ponto limite, porém persistindo ainda a possibilidade de uma bifurcação a partir dele, com a peculiaridade de que as trajetórias possuem mesma tangente em $\left(u_{0}, \lambda_{0}\right)$. Restringindo-se apenas ao caso de um ponto limite simples, um critério para a sua existência pode ser colocado na forma:

$$
\psi_{i}^{*} G_{\lambda}^{\circ} \neq 0 \quad(i=1, \ldots, m)
$$

De acordo com JEPSON \& SPENCE (1985), neste caso, se o coeficiente $a_{i j k}$ explicitado em (4.37) for diferente de zero, o ponto limite é do tipo quadrático, caso contrário é cúbico.

\subsection{2- CRITÉRIO PARA CARACTERIZAÇÃo DE PONTOS SINGULARES NA ANÁLISE ESTRUTURAL}

$\mathrm{Na}$ análise estrutural, a matriz de rigidez tangente torna-se singular nos pontos limite e de bifurcação. Logo, um ponto $q \in R^{N}$ é um ponto singular se uma das seguintes condições forem satisfeitas:

$$
\begin{aligned}
& K_{T}(q) \varphi=0 \\
& \operatorname{det} K_{T}(q)=0
\end{aligned}
$$

Em caso contrário, se det $\mathrm{K}_{\mathrm{T}}(\mathrm{q})>0$ a trajetória de equilibrio é estável em $\mathrm{q}$, enquanto que para $\operatorname{det} \mathrm{K}_{\mathrm{T}}(\mathrm{q})<0$ a trajetória de equilíbrio é instável.

Fazendo-se analogia com o estudo anterior, para definição do critério matemático para caracterização de pontos singulares em sistemas 
não-lineares, é possível definir critérios para caracterização dos mesmos em problemas estruturais. Observa-se que $G_{u}=K_{T}$ no problema estrutural, portanto pode-se associar ao vetor $\psi^{*} \circ$ autovetor $\varphi$. Sabendo-se, por outro lado, que $G_{\lambda}=-P$ os critérios para caracterização de pontos singulares na análise estrutural assumem as seguintes formas:

Pontos limite: $\varphi^{\top} P \neq 0$

Pontos de bifurcação: $\varphi^{\top} P=0$

Assim, uma vez que, durante a análise estrutural, um ponto singular é detectado através das condições (4.40), é possível diferenciar pontos limite de pontos de bifurcação utilizando-se os critérios definidos acima.

Por outro lado, a forma particular dos coeficientes expressos em (4.37) passa ser dada por:

$$
\begin{aligned}
& \mathrm{a} \equiv \varphi^{\top} \nabla_{\mathrm{q}}\left(\mathrm{K}_{\mathrm{T}} \varphi\right) \varphi \\
& \mathrm{b} \equiv \varphi^{\top} \nabla_{\lambda}\left(\mathrm{K}_{\mathrm{T}} \varphi\right)+\varphi^{\top} \nabla_{\mathrm{q}}\left(\mathrm{K}_{\mathrm{T}} \varphi\right) \overline{\mathrm{q}} \\
& \mathrm{c} \equiv \varphi^{\top} \nabla_{\lambda} \mathrm{P}+2 \varphi^{\top} \nabla_{\lambda}\left(\mathrm{K}_{\mathrm{T}} \overline{\mathrm{q}}\right)+\varphi^{\top} \nabla_{\mathrm{q}}\left(\mathrm{K}_{\mathrm{T}} \overline{\mathrm{q}}\right) \overline{\mathrm{q}} \\
& \Delta=\mathrm{b}^{2}-\mathrm{ac}
\end{aligned}
$$

onde: $\bar{q}=K_{T}^{-1} P$.

\section{6- FORMULAÇÃO DE SISTEMAS ESTENDIDOS}

$A$ adição da restrição det $K_{T}$ na equação não linear de equilibrio, dada pela equação (4.17), leva ao seguinte sistema estendido: 


$$
\bar{G}(q, \lambda)=\left\{\begin{array}{c}
G(q, \lambda) \\
\operatorname{det} K_{T}(q, \lambda)
\end{array}\right\}=0
$$

$A$ restrição det $K_{T}$ é a base para uma investigação de pontos singulares e muitas vezes a equação (4.42) é combinada com métodos do tipo comprimento de arco, resultando no seguinte sistema:

$$
\tilde{G}(q, \lambda)=\left\{\begin{array}{c}
G(q, \lambda) \\
f(q, \lambda) \\
\operatorname{det} K_{T}(q, \lambda)
\end{array}\right\}=0
$$

onde $f(q, \lambda)$ é uma equação de restrição associada ao procedimento de continuação.

Em lugar de resolver o sistema dado na equação (4.43) é possível construir um método da bisseção, no qual a direção e o comprimento do arco no método da continuação mudam de acordo com o sinal do det $\mathrm{K}_{T}$. Neste caso det $K_{T}$ verifica se na curva solução um ponto singular foi passado durante o procedimento. De acordo com WRIGGERS (1990) ○ algoritmo associado a esta situação, dado pelo quadro 4.2 , é de simples implementação. No entanto, converge linearmente para o ponto singular.

* Caminhando na trajetória de solução não-linear até det $K_{T}$ mudar de sinal

1 Faça $i=1,2, \ldots$ até convergir.

2 Mudar direção da carga e comprimento de arco: $d s_{i}=d s_{i-1} / 2$

3 Calcular nova solução com algoritmo do quadro 4.1 
3.1 Verificar o critério de convergência: se det $\mathrm{K}_{\mathrm{T}}<\mathrm{TOL} \Rightarrow$ terminar

3.2 Se o sinal de det $K_{T}$ mudar, novamente voltar ao passo 2

Senão, mudar comprimento de $\operatorname{arco:} d s_{i+1}=d s_{i} / 2$ e voltar ao passo 3.

\section{QUADRO 4.2- Algoritmo da bisseção para computar pontos singulares- WRIGGERS(1990)}

Assim, o método de Newton para o sistema de equações (4.42) pode ser obtido pela linearização da equação de restrição det $\mathrm{K}_{\mathrm{T}}=0$ de acordo com a relação dada por:

$$
\left.\frac{\mathrm{d}}{\mathrm{d} \varepsilon}\left[\operatorname{det} \mathrm{K}_{\mathrm{T}}(\mathrm{q}+\varepsilon \Delta \mathrm{q})\right)\right|_{\varepsilon=0}=\operatorname{det} \mathrm{K}_{\mathrm{T}} \operatorname{tr}\left(\mathrm{K}_{\mathrm{T}}^{-1} \nabla_{\mathrm{q}} \mathrm{K}_{\mathrm{T}} \Delta \mathrm{q}\right)
$$

Pode-se notar que o valor desta expressão requer a consideração da derivada direcional da matriz de rigidez tangente $\nabla_{\mathrm{q}} \mathrm{K}_{\mathrm{T}}$. Logo, considerando-se cargas conservativas, aplicando-se o método de Newton para a equação (4.42) resulta:

$$
\begin{aligned}
& K_{T i} \Delta q_{i+1}-P \Delta \lambda_{i+1}=-G\left(q_{i}, \lambda_{i}\right) \\
& \operatorname{tr}\left(K_{T}^{-1} \nabla_{q} K_{T} \Delta q_{i+1}\right)=-1 \\
& q_{i+1}=q_{i}+\Delta q_{i+1} \\
& \lambda_{i+1}=\lambda_{i}+\Delta \lambda_{i+1}
\end{aligned}
$$


WRIGGERS (1990) afirma que este algoritmo, não é muito ajustável para implementação em elementos finitos, visto que a operação com as duas matrizes não pode ser implementada em um padrão de elemento por elemento. De fato, a formulação da equação (4.44b) depende da derivada $\nabla_{\mathrm{q}} \mathrm{K}_{\mathrm{T}}$ que é uma operação um tanto trabalhosa.

Uma outra aproximação para a construção de sistemas estendidos, dados pela equação (4.42), para consideração de pontos singulares, baseiase no problema de autovalor:

$$
\left(\mathrm{K}_{\mathrm{T}}-\mathrm{WI}\right) \varphi=0
$$

O problema de autovalor é equivalente à restrição det $\mathrm{K}_{\mathrm{T}}=0$, visto que nos pontos singulares um autovalor $w$ de $K_{T}$ é zero, o que leva a ter $\mathrm{K}_{\mathrm{T}} \varphi=0$, que corresponde à condição dada pela equação (4.40a). Logo, o sistema estendido, dado pela equação (4.42), associado a esta restrição passa a ser dado por:

$$
\hat{G}(q, \lambda, \varphi)=\left\{\begin{array}{c}
G(q, \lambda) \\
K_{T}(q, \lambda) \varphi \\
\ell(\varphi)
\end{array}\right\}=0
$$

onde $\ell(\varphi)$ indica uma função normalizada a qual restringe a magnitude do autovetor de $K_{T}, \varphi \in R^{N}$, no ponto singular. Neste caso, a expressão escolhida para $\ell(\varphi)$ é dada por:

$$
\ell(\varphi)=\|\varphi\|-1=0
$$

Os sistemas estendidos dados pela equação (4.46) estão associados com $2 n+1$ incógnitas e desta maneira o esforço numérico para resolver tais sistemas é aparentemente maior, porém esta dificuldade pode ser contornada com o uso do método da partição. 
Com o objetivo de desenvolver um método eficiente para cálculo de pontos singulares utilizando-se (4.46), é possível derivar um método iterativo do tipo Newton com convergência quadrática.

\section{7- MÉTOdO DE NEWTON PARA SOLUÇÃO DE SISTEMAS ESTENDIDOS}

O sistema de solução incremental, para equações não-lineares, utilizando-se método de Newton leva à seguinte formulação:

$$
\begin{aligned}
& \hat{\mathrm{K}}_{\mathrm{Ti}} \Delta \mathrm{d}_{\mathrm{i}}=-\hat{\mathrm{G}}\left(\mathrm{d}_{\mathrm{i}}\right) \\
& \mathrm{d}_{\mathrm{i}+1}=\mathrm{d}_{\mathrm{i}}+\Delta \mathrm{d}_{\mathrm{i}}
\end{aligned}
$$

onde $\hat{K}_{T i} \Delta d_{i}=\left.\frac{d}{d \varepsilon}\left[\hat{G}\left(d_{i}+\varepsilon \Delta d_{i}\right)\right]\right|_{\varepsilon=0} e d_{i}=\left\{\begin{array}{l}q \\ \lambda \\ \varphi\end{array}\right\}_{i}$.

Assim, da linearização da equação (4.46) resulta:

$$
\left[\begin{array}{ccc}
\mathrm{K}_{T} & 0 & -\mathrm{P} \\
\nabla_{\mathrm{q}}\left(\mathrm{K}_{\mathrm{T}} \varphi\right) & \mathrm{K}_{\mathrm{T}} & \nabla_{\lambda}\left(\mathrm{K}_{T} \varphi\right) \\
0^{\top} & \frac{\varphi^{\top}}{\|\varphi\|} & 0
\end{array}\right]\left\{\begin{array}{c}
\Delta q \\
\Delta \varphi \\
\Delta \lambda
\end{array}\right\}=-\left\{\begin{array}{c}
\mathrm{G}(\mathrm{q}, \lambda) \\
\mathrm{K}_{T}(\mathrm{q}, \lambda) \varphi \\
\|\varphi\|-1
\end{array}\right\}
$$

onde as derivadas direcionais de $\mathrm{K}_{\mathrm{T}}$ são dadas pelas equações:

$$
\begin{aligned}
& \nabla_{\mathrm{q}}\left(\mathrm{K}_{T} \varphi\right) \Delta q=\left.\frac{\mathrm{d}}{\mathrm{d} \varepsilon}\left[\mathrm{K}_{\mathrm{T}}(\mathrm{q}+\varepsilon \Delta \mathrm{q}, \lambda) \varphi\right]\right|_{\varepsilon=0} \\
& \nabla_{\lambda}\left(\mathrm{K}_{T} \varphi\right) \Delta \lambda=\left.\frac{\mathrm{d}}{\mathrm{d} \varepsilon}\left[\mathrm{K}_{T}(\mathrm{q}, \lambda+\varepsilon \Delta \lambda) \varphi\right]\right|_{\varepsilon=0}
\end{aligned}
$$


Utilizando-se o método da partição para resolução do sistema de equações dado pela equação (4.49), tem-se que: da primeira equação de (4.49) o incremento do deslocamento é dado por $\Delta q=\Delta \lambda \Delta q_{P}+\Delta q_{G}$, e substituindo-o na segunda obtém-se:

$$
\mathrm{K}_{\mathrm{T}} \Delta \varphi=-\Delta \lambda\left[\nabla_{\mathrm{q}}\left(\mathrm{K}_{\mathrm{T}} \varphi\right) \Delta \mathrm{q}_{\mathrm{P}}+\nabla_{\lambda}\left(\mathrm{K}_{\mathrm{T}} \varphi\right)\right]-\left[\nabla_{\mathrm{q}}\left(\mathrm{K}_{\mathrm{T}} \varphi\right) \Delta \mathrm{q}_{\mathrm{G}}+\mathrm{K}_{\mathrm{T}} \varphi\right]
$$

Deste modo, definindo-se os vetores $h_{1}$ e $h_{2}$ por:

$$
\begin{aligned}
& \mathrm{h}_{1}=\nabla_{\mathrm{q}}\left(\mathrm{K}_{\mathrm{T}} \varphi\right) \Delta \mathrm{q}_{\mathrm{p}}+\nabla_{\lambda}\left(\mathrm{K}_{\mathrm{T}} \varphi\right) \\
& \mathrm{h}_{2}=\nabla_{\mathrm{q}}\left(\mathrm{K}_{\mathrm{T}} \varphi\right) \Delta \mathrm{q}_{\mathrm{G}}+\mathrm{K}_{\mathrm{T}} \varphi,
\end{aligned}
$$

tem-se que o incremento total do autovetor $\varphi$ é dado por:

$$
\Delta \varphi=\Delta \lambda \Delta \varphi_{1}+\Delta \varphi_{2}
$$

onde:

$$
\Delta \varphi_{1}=-h_{1} K_{T}^{-1} \text { e } \Delta \varphi_{2}=-h_{2} K_{T}^{-1}
$$

Assim, levando-se a expressão de $\Delta \varphi$ na terceira de (4.49) resulta:

$$
\Delta \lambda=\frac{-\varphi^{\top} \Delta \varphi_{2}+\|\varphi\|-\|\varphi\|^{2}}{\varphi^{\top} \Delta \varphi_{1}}
$$

Em problemas onde a carga é independente da deformação (carga conservativa), por exemplo carga permanente, o termo $\nabla_{\lambda}\left(K_{T} \varphi\right)$ é igual a zero.

Então, com o sistema resolvido, pode-se construir um algoritmo para o procedimento de solução da equação (4.49), que corresponde à equação 
(4.46) linearizada. Considerando-se apenas um passo de iteração, o procedimento é apresentado no quadro 4.3.

1 Resolver:

$$
\begin{aligned}
& \mathrm{K}_{\mathrm{T}} \Delta \mathrm{q}_{\mathrm{P}}=\mathrm{P} \\
& \mathrm{K}_{\mathrm{T}} \Delta \mathrm{q}_{\mathrm{G}}=-\mathrm{G}
\end{aligned}
$$

2 Calcular dentro de um "loop" para todos os elementos

$$
\begin{aligned}
& \mathrm{h}_{1}=\nabla_{\mathrm{q}}\left(\mathrm{K}_{\mathrm{T}} \varphi\right) \Delta \mathrm{q}_{\mathrm{P}}+\nabla_{\lambda}\left(\mathrm{K}_{\mathrm{T}} \varphi\right) \\
& \mathrm{h}_{2}=\mathrm{K}_{\mathrm{T}} \varphi+\nabla_{\mathrm{q}}\left(\mathrm{K}_{\mathrm{T}} \varphi\right) \Delta \mathrm{q}_{\mathrm{G}}
\end{aligned}
$$

3 Resolver

$$
\begin{aligned}
& \mathrm{K}_{\mathrm{T}} \Delta \varphi_{1}=-\mathrm{h}_{1} \\
& \mathrm{~K}_{\mathrm{T}} \Delta \varphi_{2}=-\mathrm{h}_{2}
\end{aligned}
$$

4 Calcular novos incrementos

$$
\begin{aligned}
& \Delta \lambda=\frac{-\varphi^{\top} \Delta \varphi_{2}+\|\varphi\|-\|\varphi\|^{2}}{\varphi^{\top} \Delta \varphi_{1}} \\
& \Delta q=\Delta \lambda \Delta q_{P}+\Delta q_{G} \\
& \Delta \varphi=\Delta \lambda \Delta \varphi_{1}+\Delta \varphi_{2}
\end{aligned}
$$

5 Atualizar deslocamentos, autovetores e parâmetro de carga

$$
\begin{aligned}
& q=q+\Delta q \\
& \varphi=\varphi+\Delta \varphi \\
& \lambda=\lambda+\Delta \lambda
\end{aligned}
$$


Para construir uma aproximação numérica que conserve a taxa quadrática de convergência do Método de Newton, tem-se que o vetor $K_{T} \varphi$ pode ser representado pela derivada direcional do resíduo $G$ na direção de $\varphi$, de acordo com a seguinte expressão:

$$
\mathrm{K}_{\mathrm{T}} \varphi=\nabla_{\mathrm{q}} \mathrm{G}(\mathrm{q}, \lambda) \varphi=\left.\frac{\mathrm{d}}{\mathrm{d} \varepsilon} \mathrm{G}(\mathrm{q}+\varepsilon \varphi, \lambda)\right|_{\varepsilon=0}
$$

Explorando-se a simetria da segunda derivada de G e o fato de $\varphi$ e $\Delta q$ serem vetores independentes, pode-se expressar a derivada direcional de $\mathrm{K}_{T} \varphi$ na direção de $\Delta \mathrm{q}$ da seguinte forma:

$$
\begin{aligned}
\nabla_{\mathrm{q}}\left(\mathrm{K}_{\mathrm{T}} \varphi\right) \Delta \mathrm{q} & =\nabla_{\mathrm{q}}\left[\nabla_{\mathrm{q}} \mathrm{G}(\mathrm{q}, \lambda) \varphi\right] \Delta \mathrm{q} \\
& =\nabla_{\mathrm{q}}\left[\nabla_{\mathrm{q}} \mathrm{G}(\mathrm{q}, \lambda) \Delta \mathrm{q}\right] \varphi
\end{aligned}
$$

Assim, pode-se utilizar a definição de derivada direcional para obter a seguinte expressão que substitui a (4.50):

$$
\nabla_{\mathrm{q}}\left[\mathrm{K}_{\mathrm{T}} \varphi\right] \Delta \mathrm{q}=\left.\frac{\mathrm{d}}{\mathrm{d} \varepsilon}\left[\mathrm{K}_{\mathrm{T}}(\mathrm{q}+\varepsilon \varphi)\right] \Delta \mathrm{q}\right|_{\varepsilon=0}
$$

A equação acima pode ser escrita num formato alternativo, mais agradável para aproximação numérica, dado por:

$$
\nabla_{\mathrm{q}}\left[\mathrm{K}_{\mathrm{T}} \varphi\right] \Delta \mathrm{q}=\lim _{\varepsilon \rightarrow 0} \frac{1}{\varepsilon}\left[\mathrm{K}_{\mathrm{T}}(\mathrm{q}+\varepsilon \varphi) \Delta \mathrm{q}-\mathrm{K}_{\mathrm{T}}(\mathrm{q}) \Delta \mathrm{q}\right]
$$

Com uma escolha adequada do parâmetro $\varepsilon$, obtém-se:

$$
\nabla_{\mathrm{q}}\left[\mathrm{K}_{\mathrm{T}} \varphi\right] \Delta \mathrm{q} \cong \frac{1}{\varepsilon}\left[\mathrm{K}_{\mathrm{T}}(\mathrm{q}+\varepsilon \varphi) \Delta \mathrm{q}-\mathrm{K}_{\mathrm{T}}(\mathrm{q}) \Delta \mathrm{q}\right]
$$


A aplicação desta aproximação para a derivada direcional, no algoritmo do quadro 4.3 leva às seguintes expressões para os vetores $h_{1}$ e $h_{2}$ :

$$
\begin{aligned}
& \mathrm{h}_{1} \cong \frac{1}{\varepsilon}\left[K_{T}(q+\varepsilon \varphi) \Delta q_{P}-P\right] \\
& h_{2} \cong \frac{1}{\varepsilon}\left[K_{T}(q+\varepsilon \varphi) \Delta q_{G}+G\right]+K_{T} \varphi
\end{aligned}
$$

Observa-se que neste estágio, $P$ e $G$ já estão calculados. Consequentemente, a consideração de $h_{i}(i=1,2)$ através das expressões dadas pela equação (4.58) envolve somente um cálculo adicional da matriz de rigidez, isto é, $K_{T}(q+\varepsilon \varphi)$.

Segundo WRIGGERS (1990), a escolha adequada do parâmetro $\varepsilon$ em (4.58) é fundamental para o sucesso do método. Esta escolha depende dos vetores q e $\varphi$ e da precisão do computador. Uma estimativa para $\varepsilon$ pode ser encontrada em DENNIS (1983) ${ }^{1}$ apud WRIGGERS (1990) que propõe a seguinte fórmula:

$$
\varepsilon=\max _{K K \mathrm{~K}<\mathrm{n}} q_{K} \eta_{\mathrm{TOL}}
$$

onde $q_{k}$ é a k-ésima componente do vetor deslocamento, $q \in R^{N}$, e $\eta_{T O L}$ é uma constante de precisão da máquina.

\section{8- ALGORITMO GERAL PARA CONSIDERAÇÃo DA ESTABILIDADE EM PROBLEMAS NÃO-LINEARES}

\footnotetext{
1 DENNIS J.E.; SCHNABEL R.B. (1983). Numerical Methods for Unconstrained Optimization and Nonlinear Equations, Prentice Hall, Englewood Cliffs, New Jersey.
} 
Visto que os sistemas estendidos fornecem uma ferramenta para cálculo direto de pontos singulares, é vantajoso combiná-los com os procedimentos de continuação. Dentro deste algoritmo geral, um método de comprimento de arco é usado para seguir no ramo de solução. Portanto, é necessário ligarem os dois processos de solução.

Uma aproximação simples a ser empregada baseia-se na inspeção do determinante da matriz de rigidez tangente, det $\mathrm{K}_{\mathrm{T}}$. Se o determinante de $\mathrm{K}_{\mathrm{T}}$ passar por um ponto de máximo ou mínimo, pode-se ligar o método comprimento de arco ao sistema estendido para computar um ponto singular próximo na curva carga/deslocamento. Desta maneira passos grandes comparados com um método de comprimento de arco são possíveis, ver figura 4.10 .

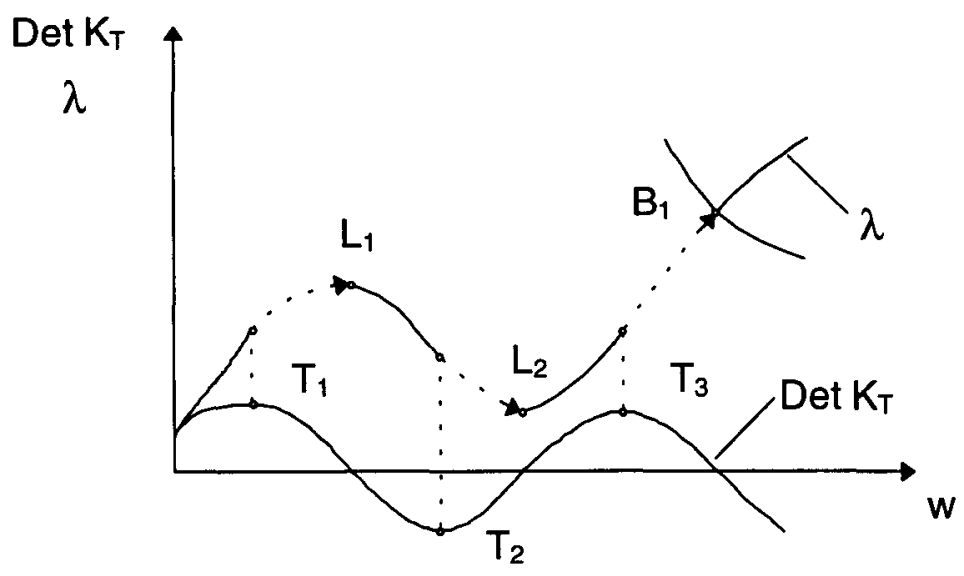

FIGURA 4.10- Inspeção do det $K_{T}$

Quando se liga o método de comprimento de arco ao sistema estendido necessita-se de um valor inicial para o vetor $\varphi$, que representa 0 autovetor de $K_{T}$ no ponto singular. Segundo WRIGGERS (1990), a melhor escolha para $\varphi$ é o autovetor de $\mathrm{K}_{\mathrm{T}}$ no estado atual, sendo calculado por uma iteração inversa. Sabendo-se que este método frequentemente é de convergência rápida, o primeiro passo desta iteração produz um vetor $\varphi \circ$ 
qual é de exatidão suficiente. Portanto esta operação requer somente uma substituição no passo anterior. Se pontos limites são esperados, uma boa aproximação para $\varphi$ é o campo dos deslocamentos, $q_{(1)}$, do primeiro passo de Newton.

Deste modo, uma vez que $\varphi$ é conhecido torna-se fácil caracterizar os pontos singulares utilizando-se o critério dado pela equação (4.41).

Portanto um algoritmo geral para caracterização de pontos singulares nas curvas não-lineares carga/deslocamento pode ser construído, como mostra o quadro 4.4. Assim, iniciando-se de um ponto singular o procedimento comprimento de arco pode ser usado para seguir o ramo póscrítico. No caso de pontos de bifurcação um procedimento de mudança de ramo é exigido se um ramo secundário for investigado. Para este objetivo o autovetor $\varphi$ associado com a carga de flambagem é necessário, sendo este considerado automaticamente pelo sistema estendido. Portanto, se inicialmente sabe-se que se trata de um problema de bifurcação simples, não é necessário uma solução adicional de um problema de autovalor; pode-se partir de um estado inicial já deslocado, como será mostrado no capítulo 5. Os problemas de barras e chapas comprimidas e cascas em regime de membrana são casos que apresentam problema de bifurcação simples.

O quadro abaixo, mostra o algoritmo geral para caracterização de pontos singulares.

1 Cálculo dos estados de equilíbrio com o método de comprimento de arco ao longo da trajetória de solução, utilizando-se o algoritmo do quadro 4.1

$$
G(q, \lambda)=R(q)-\lambda P
$$

2 Observar o determinante de $\mathrm{K}_{\mathrm{T}}$ 
2.1 Se o determinante passar por um máximo ou mínimo: ir ao passo 3

3 Consideração direta do ponto singular

3.1 Cálculo do vetor $\varphi$ via primeiro passo de uma iteração inversa:

$$
\varphi_{\circ}=\mathrm{K}_{\top}^{-1} \mathrm{I}
$$

3.2 Empregar o método de Newton para resolver o sistema estendido, usando o algoritmo do quadro 4.2

$$
\hat{\mathrm{G}}(q, \lambda, \varphi)=0
$$

4 Critério para caracterizar o ponto singular

$$
\begin{array}{ll}
\varphi^{\top} P \neq 0 \rightarrow \text { ponto limite: } & \text { voltar ao passo } 1 \\
\varphi^{\top} P=0 \rightarrow \text { ponto de bifurcação: } & \text { ir ao passo } 5
\end{array}
$$

5 Ponto de bifurcação, usar algoritmo para procedimento de mudança de ramo

5.1 Salvar informação para reiniciar o problema

5.2 Se o cálculo do ramo secundário é desejado: voltar ao passo 1

5.3 Senão, se o sistema estendido não for usado, resolver problema de autovalor:

$$
\left(K_{T}-w I\right) \varphi=0
$$

5.4 Adicionar autovetor $\varphi$ ao vetor deslocamento $\bar{q}$, onde $\bar{q}$ é definido por: $\bar{q}=K_{T}^{-1} P$ 


$$
q=\bar{q}+\xi \frac{\varphi}{\|\varphi\|}
$$

5.5 Calcular um estado de equilíbrio no ramo secundário usando método comprimento de arco

$$
G(q \lambda)=R(q)-\lambda P=0
$$

5.6 Se o ramo secundário é alcançado: ir ao passo 6

5.7 Senão escolher novo fator escalar $\xi$ : voltar ao passo 5.4

6 Cálculo dos estados de equilíbrio no ramo secundário usando método comprimento de arco

7 Se outro ramo tem que ser calculado: Recomeçar e voltar ao passo 5.2

8 Se todos os ramos foram calculados: terminar

QUADRO 4.4- Algoritmo para cálculo de pontos singulares e de ramos secundários WRIGGERS (1990) 


\section{CAPÍTULO 5- EXEMPLOS DE APLICAÇÃO}

Uma vez que o estudo apresentado anteriormente já se encontra em parte implementado em códigos de cálculo no sistema LUSAS, os exemplos apresentados nesta seção são gerados empregando-se este sistema. Os elementos finitos utilizados estão disponíveis em LUSAS (1995).

\section{1- EXEMPLO DE NLG EM TRELIÇA ESPACIAL}

A estrutura considerada neste exemplo é uma treliça espacial de base quadrada, com lado igual a $4.0 \mathrm{~m}$ e altura total de $20.0 \mathrm{~m}$, apresentada em RUBERT (1993). As barras que formam as colunas têm área da seção transversal igual a $35.0 \mathrm{~cm}^{2}$ e as demais $7.5 \mathrm{~cm}^{2}$; o material empregado possui módulo de elasticidade $E=21000 \mathrm{kN} / \mathrm{cm}^{2}$. A treliça é simétrica, sendo uma face típica ilustrada pela figura 5.1c. O carregamento total aplicado é formado por uma força vertical de $3000.0 \mathrm{kN}$ e outra horizontal de $150 \mathrm{kN}$ no ponto 9, nos pontos 6 e 8 uma força horizontal de $37.5 \mathrm{kN}$, como mostrado na figura 5.1c.

Com o objetivo de exemplificar o efeito não-linear geométrico realizaram-se para a estrutura acima descrita duas análises, uma linear $e$ outra não-linear geométrica. 

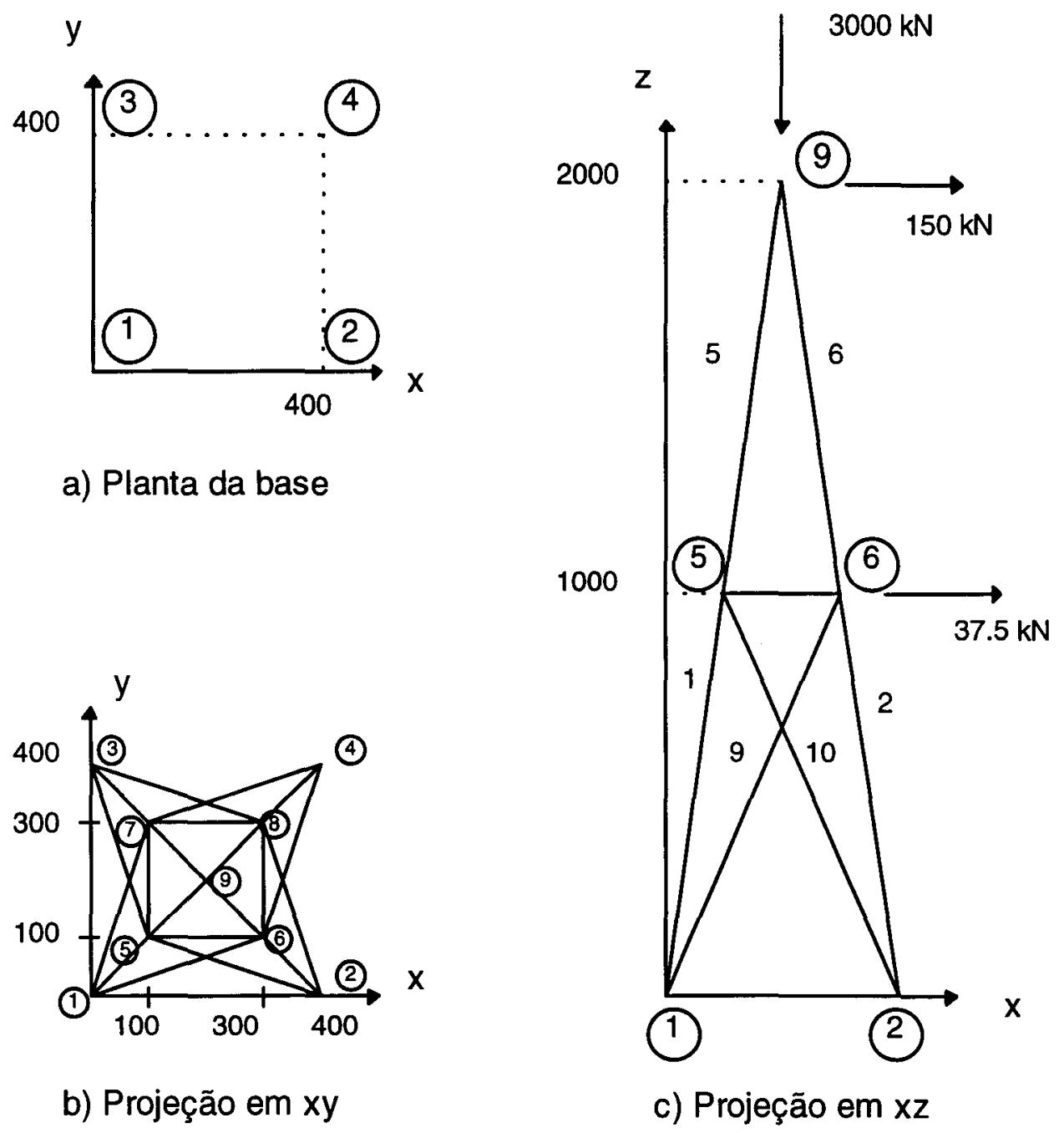

FIGURA 5.1- Treliça espacial de base quadrada - RUBERT (1993)

Nas duas análises a estrutura foi discretizada empregando-se o elemento finito de barra em três dimensões, BRS2, que possui 3 graus de liberdade por nó, que são os deslocamentos na direção x, y e z.

$\mathrm{Na}$ análise não-linear utilizou-se a formulação Lagrangiana Total, uma vez que a Lagrangiana Atualizada não é disponível no LUSAS (1995) para o elemento finito empregado. $O$ procedimento adotado para solução do sistema de equações não-lineares foi o de Newton-Raphson clássico. 0 critério de convergência adotado em cada iteração foi baseados nas normas 
de deslocamento e de forças residuais, ambas limitadas em $1 \%$. 0 carregamento total foi aplicado em cem etapas.

Os resultados obtidos em termos de trajetória de equilíbrio estão apresentados na figura 5.2 .

As curvas carga vertical/deslocamento horizontal para o ponto 9 correspondentes às análises linear e não-linear, permitem verificar o efeito não-linear geométrico. Pode-se observar que o deslocamento do ponto 9 é aproximadamente $85 \%$ maior do que o obtido na análise linear.

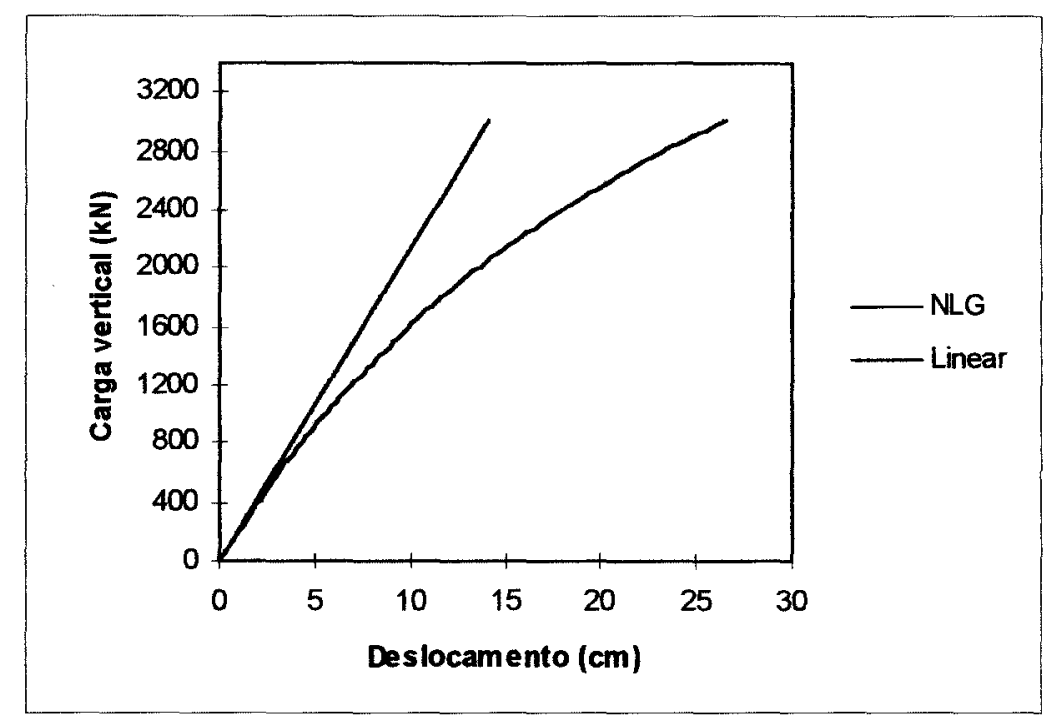

FIGURA 5.2- Deslocamento horizontal do ponto 9

\section{2- EXEMPLO DE CARGA LIMITE - TRELIÇA SIMPLES}

Neste caso, a estrutura considerada consiste de uma treliça plana de uma barra, cujas características geométricas e do material estão apresentadas na figura 5.3 . 


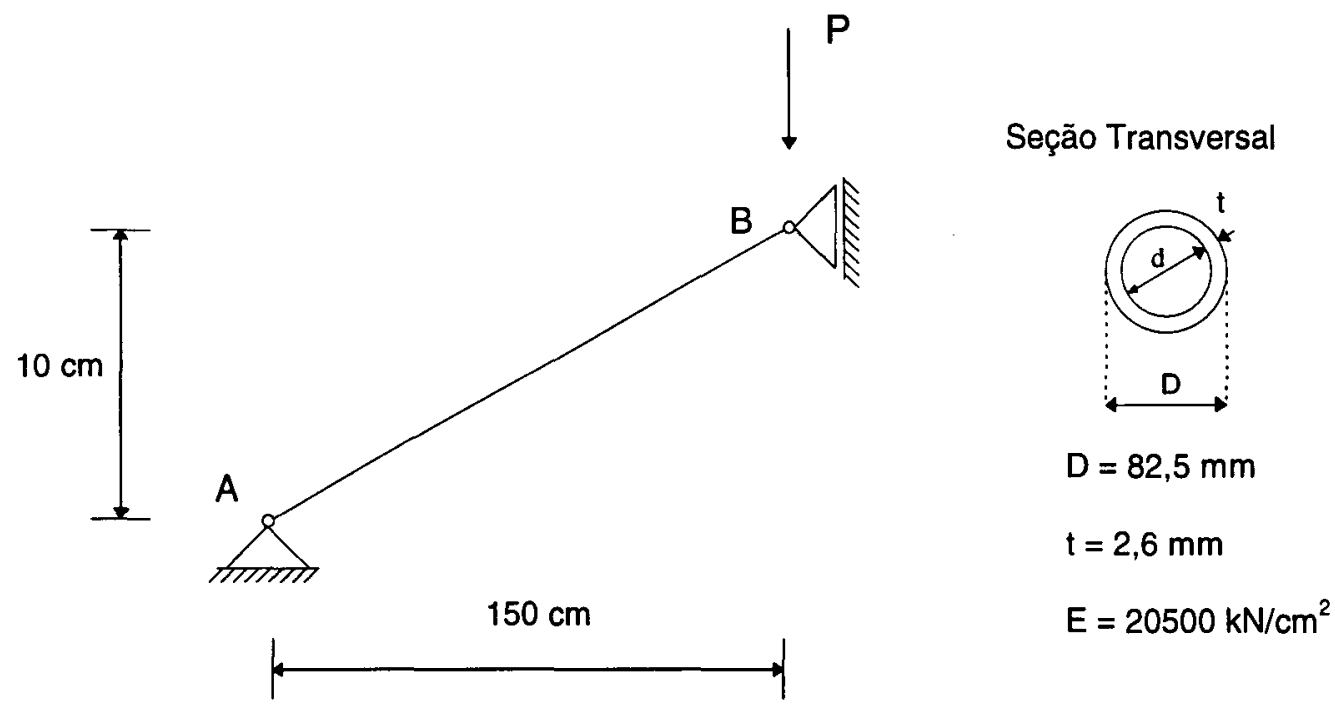

FIGURA 5.3- Treliça simples

Neste exemplo realizaram-se duas análises: uma considerando deslocamento controlado e outra com controle de carga no processo incremental-iterativo.

$\mathrm{Na}$ análise com deslocamento controlado empregou-se um elemento finito bidimensional isoparamétrico de barra, (BAR3), com 3 nós e dois graus de liberdade em deslocamentos por nó. Nesta análise o controle foi sobre o deslocamento vertical do ponto $B$, mostrado na figura 5.3. $O$ incremento inicial do deslocamento do ponto $B$ foi de $0,5 \mathrm{~cm}$.

$\mathrm{Na}$ análise com carregamento imposto empregou-se um elemento finito isoparamétrico de barra de eixo reto, (BAR2), com 2 nós e 2 graus de liberdade em deslocamentos por nó. $O$ incremento de carga inicial foi de $0,4 \mathrm{kN}$ na direção de $P$.

Nas duas análises empregou-se a formulação Lagrangiana Total e o método de Newton-Raphson clássico. $O$ critério de convergência adotado foi o da norma dos deslocamento limitada em $0,1 \%$ e de forças residuais em $1 \%$. 


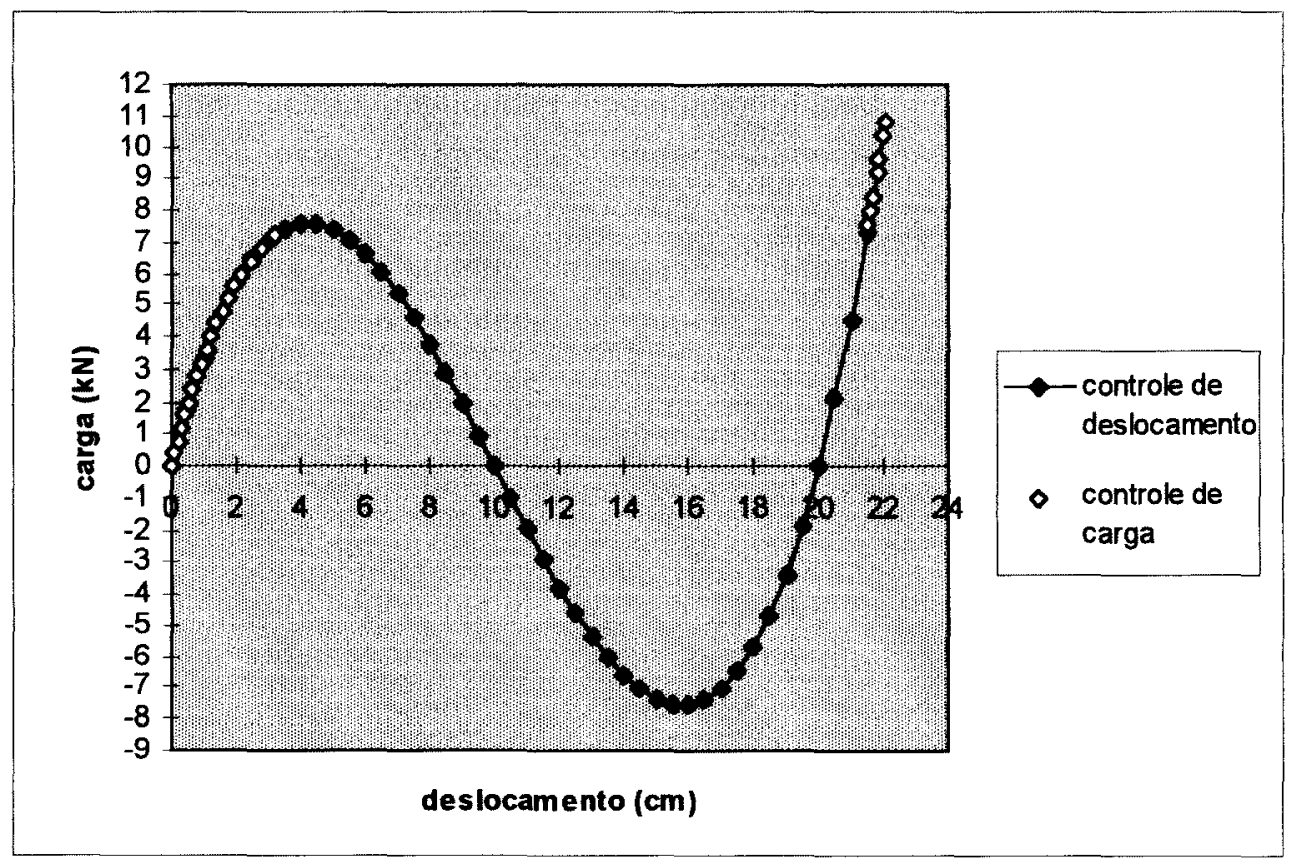

FIGURA 5.4- Deslocamento vertical do ponto B

A figura 5.4 apresenta o resultado das duas análises em questão para o deslocamento vertical do ponto B limitado em $22 \mathrm{~cm}$.. Em particular observa-se o comportamento "snap through" na curva carga/deslocamento quando a carga crítica foi atingida no caso de controle de carga. $\mathrm{Na}$ situação de deslocamento controlado, ao contrário, a curva carga/deslocamento é completa.

Observa-se também que o resultado obtido na análise com deslocamento controlado é idêntico ao analítico apresentado no capítulo 4.

\section{3- EXEMPLO DE CARGA CRÍTICA - BARRA COMPRIMIDA AXIALMENTE}

A estrutura considerada consiste de uma barra esbelta submetida a uma carga $\mathrm{P}$ centrada, de compressão. A barra possui área transversal de $1.0 \mathrm{~cm}^{2}$, comprimento inicial de $2.5 \mathrm{~m}$ e material com módulo de elasticidade $E=20500 \mathrm{kN} / \mathrm{cm}^{2}$. 


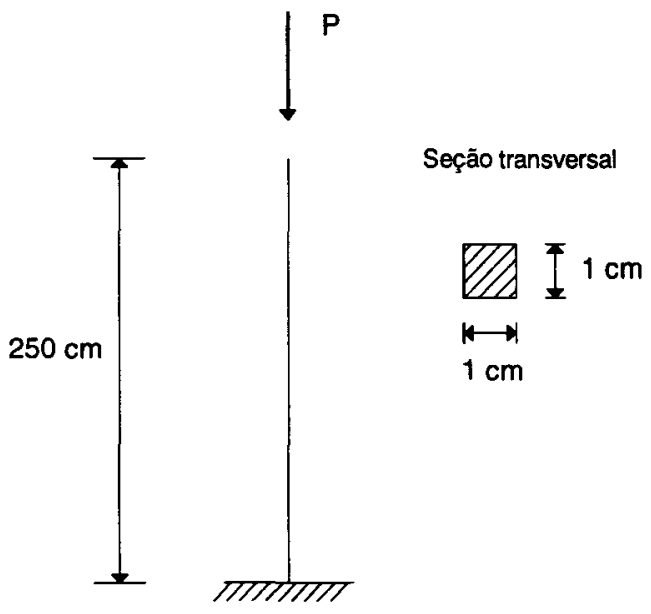

FIGURA 5.5- Barra sujeita à compressão centrada

Neste exemplo realizaram-se duas análises, a primeira considerando a barra perfeitamente na vertical e a segunda admitindo-se uma certa imperfeição inicial representada por uma inclinação de aproximadamente $0.23^{\circ}$ com a vertical.

Nas duas análises planas, empregou-se o elemento finito de viga, com 3 nós e 3 graus de liberdade por nó (dois deslocamentos independentes no plano da barra e uma rotação no. plano). A formulação utilizada foi a Lagrangiana Atualizada. $O$ incremento inicial de carga foi $10 \mathrm{kN}$.

A análise sem imperfeição inicial, apresenta um ponto de bifurcação quando a carga aplicada atinge o valor crítico. Neste caso, no procedimento de solução numérica, deve-se utilizar um algoritmo semelhante ao apresentado no quadro 4.4 do capítulo 4 , de modo que se possa determinar o ponto de bifurcação e a partir deste prosseguir a simulação num ramo secundário se for de interesse. O sistema LUSAS permite realizar este procedimento. Neste exemplo, o método utilizado para encontrar o ponto de bifurcação foi o da bisseção, apresentado anteriormente. 
É importante observar que a análise pós-crítica ou no ramo secundário de equilíbrio somente é possivel se o ramo primário estiver determinado, isto é se o valor crítico for conhecido.

No ramo primário de equiliíbrio adotou-se um fator inicial de carregamento igual a 1 , com norma dos deslocamentos fixada em $0,01 \%$. No ramo secundário, o valor inicial do comprimento de arco foi 0,005 , o fator inicial de carregamento foi 6,8749 que é o valor correspondente ao fator de carga no ponto de bifurcação na análise do ramo primário. $O$ critério de parada foi fator de carga máximo de 50 ou número máximo de incrementos igual a 800 .

A análise, considerando-se uma imperfeição inicial, foi realizada com objetivo de ilustrar o caso em que se sabe a priori que o problema é de bifurcação simples (no sistema sem imperfeição) e que neste caso, pode-se partir de um estado já deslocado e realizar apenas uma análise não-linear geométrica simples. $O$ incremento de carga inicial foi de $2 \mathrm{kN}$, o critério de convergência foi o da verificação da norma dos deslocamentos limitada em $0,01 \%$.

A figura 5.6 ilustra graficamente, em forma adimensional, os resultados obtidos. No eixo vertical estão os valores da carga aplicada dividida pelo valor da carga crítica, no caso $68,458 \mathrm{~N}$, e no eixo horizontal os valores do deslocamento horizontal do extremo superior da barra dividido pelo comprimento inicial. 


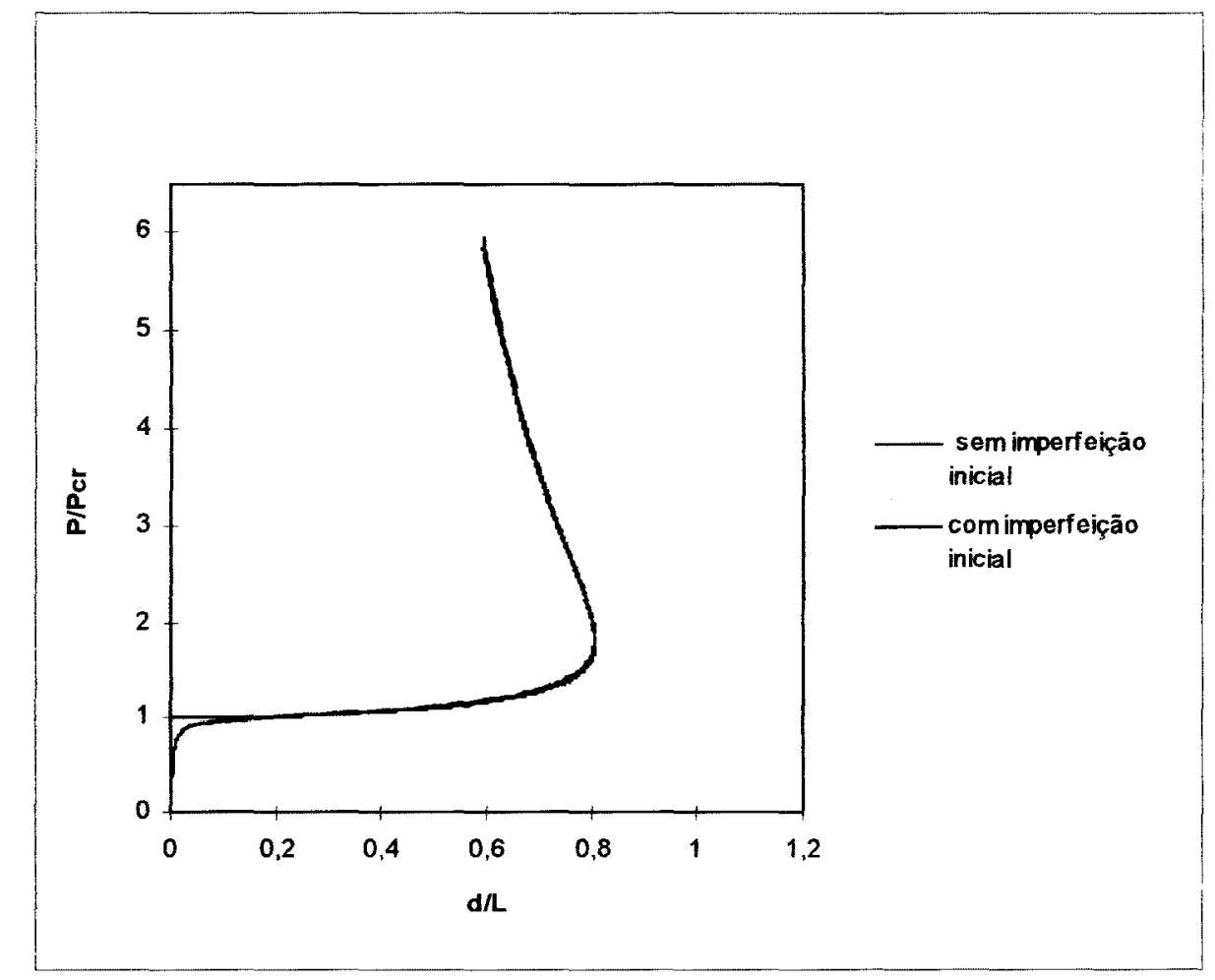

FIGURA 5.6- Comportamento não-linear geométrico de uma barra sujeita à compressão centrada 


\section{CAPÍTULO 6- CONSIDERAÇÕES FINAIS E CONCLUSÕES}

Neste trabalho são apresentados os conceitos teóricos envolvidos no estudo do comportamento não-linear geométrico das estruturas, utilizandose o Princípio dos Trabalhos Virtuais em combinação com o Método dos Elementos Finitos.

A descrição do movimento das partículas de um meio contínuo pode ser feita tomando-se como referência as configurações indeformada (inicialmente ocupada) ou deformada (atualmente ocupada). Quando a descrição se refere à configuração indeformada ela é dita material e quando se refere à deformada é dita espacial.

Em função da descrição adotada para o movimento obtêm-se as diferentes formas para a representação do equilíbrio da estrutura na posição deslocada. Isto é, ao utilizar-se a descrição material a formulação obtida é chamada de Lagrangiana; já a partir da descrição espacial obtém-se a formulação Euleriana. No caso da análise estrutural não-linear geométrica, a formulação Lagrangiana é mais conveniente, visto que a configuração deformada é desconhecida. Deste modo, numa análise numérica uma maneira de aproximar-se da formulação Euleriana é realizar uma atualização contínua da configuração de referência, em intervalos de tempos muito pequenos, que constitui a chamada formulação Lagrangiana Atualizada. 
De modo mais específico, no âmbito de um procedimento incremental-iterativo, na descrição Lagrangiana Atualizada a configuração de referência pode ser tanto a última configuração equilibrada obtida no final de cada passo de carga, quanto a configuração correspondente à última iteração. Aliás, em diversas referências bibliográficas a Formulação Lagrangiana Atualizada, com atualização da configuração de referência a cada iteração, tal como a apresentada em WRIGGERS (1990) e no sistema LUSAS, é normalmente denominada como forma Euleriana.

Observou-se também, que a formulação Lagrangiana Total é a mais utilizada nas análises, apesar de verificar-se neste trabalho que a expressão da matriz de rigidez tangente é mais simples quando se emprega a formulação Lagrangiana Atualizada.

A questão da estabilidade do equilíbrio foi tratada neste trabalho com o objetivo de esclarecer os critérios matemáticos encontrados na literatura, para conceituação e caracterização de pontos singulares. Um outro aspecto de interesse abordado foi relativo à implementação desses critérios em algoritmos para análise numérica. Para tal, utilizou-se como referência o texto apresentado em WRIGGERS (1990) e suas indicações.

Embora as formulações estudadas neste trabalho não tenham sido implementadas em código de cálculo, por uma questão de prazo para sua conclusão, os exemplos gerados no sistema LUSAS permitiram ilustrar de forma satisfatória os conceitos apresentados.

O exemplo 1 ilustrou o efeito da consideração da não-linearidade geométrica na análise do comportamento de uma treliça espacial, comparando-se o resultado com o de uma análise linear. Verificou-se, neste caso, que o deslocamento horizontal do topo da treliça na análise não-linear foi $85 \%$ maior do que o obtido na análise linear. 
O exemplo 2 procurou dar ênfase ao conceito de ponto limite procurando determinar a carga limite de uma treliça plana simples. Neste exemplo realizaram-se duas análises não-lineares, uma considerando-se um procedimento com deslocamentos controlados e a outra com carga controlada. Através do controle de carga verificou-se que a curva carga/deslocamento apresenta um salto quando a carga atinge o valor da carga limite. O mesmo não ocorre no caso de deslocamentos controlados. Nesse caso, é possível descrever toda a curva carga/deslocamento inclusive em seu ramo instável.

O exemplo 3 ilustrou o conceito de ponto de bifurcação para uma barra sujeita à compressão centrada. Realizaram-se neste exemplo duas análises, uma considerando a barra totalmente na vertical e outra com pequena imperfeição inicial, isto é inclinação de aproximadamente $0.23^{\circ}$ com a vertical. No primeiro caso é necessário resolver problema de autovalor e utilizar métodos para continuação da análise no ramo secundário. No segundo, realiza-se apenas uma análise não-linear geométrica completa.

De um modo geral, pode-se afirmar que o objetivo proposto inicialmente foi alcançado com êxito.

Como sugestões para continuidade deste trabalho destacam-se: a implementação em código de cálculo dos algoritmos apresentados, a consideração de grandes deformações no âmbito da elasticidade não-linear e aplicação visando a análise de outros tipos de sistemas estruturais. 


\section{REFERÊNCIAS BIBLIOGRÁFICAS}

BATHE, K.-J. (1982). Finite element procedures in engineering analysis. Englewood Cliffs, Prentice Hall.

BERGAN, P.G. (1980). Solution algorithms for nonlinear structural problems. Computers and Structures, v.12, p.497-509.

BREZZI, F. (1981). Behaviour of finite element solutions near a bifurcation point. In: WUNDERLICH, W.; STEIN, E.; BATHE, K.-J., eds. Nonlinear finite element analysis in structural mechanics. Berlin, Springer-Verlag. p.109-121.

CARRERA, E. (1994). A study on arc-length-type methods and their operation failures illustrated by a simple model. Computers and Structures, v.50, n.2, p.217-229.

CESCOTTO, S.; FREY, F.; FONDER, G. (1979). Total and updated lagrangian descriptions in nonlinear structural analysis: a unified approach. In: GLOWINSKI, R.; RODIN, E.Y.; ZIENKIEWICS, O.C., eds. Energy methods in finite element analysis. Chichester, John Wiley, p.283-296. 
CHEN, C.-N. (1996). A finite element study on bifurcation and limit point buckling of elastic-plastic arches. Computers and Structures, v.60, p.189-196.

COIMBRA, A.L. (1981). Novas lições de mecânica do contínuo. ed. Edgard Blücher Ltda.

CORRÊA, M.R.S. (1991). Aperfeiçoamento de modelos usualmente empregados no projeto de sistemas estruturais de edifícios. São Carlos. Tese (Doutorado)- Escola de Engenharia de São Carlos, Universidade de São Paulo.

CRANDALL, M.G.; RABINOWITZ, P.H. (1973). Bifurcation, pertubation of simple eigenvalues, and linearized stability. Archive Rational Mechanics Analysis. v.52, p.161-180.

CRISFIELD, M.A. (1991). A fast incremental/iterative solution procedure that handles "snap-through". Computers and Structures, v.13, p.55-62.

CRISFIELD, M.A. (1991). Non-linear finite element analysis of solids and structures. New York, John Wiley. v.1

DECKER, D.W.; KELLER, H.B. (1980). Multiple limit point bifurcation. Journal of Mathematical Analysis and Applications, v.75, p.417-430.

DECKER, D.W.; KELLER, H.B. (1981). Convergence acceleration for newton's method at singular points.. SIAM Journal Numerical Analysis, v.19, n.1, p.219-229.

DELL'ACQUA, L.C. (1978). Instabilita'delle strutture. 2.ed. Milano, CLUP. 
DIOGO, L.A.C. (1988). Sobre a matriz de rigidez tangente das barras de treliças planas sujeitas a rotações grandes. Anais EPUSP, Engenharia Civil, Engenharia de Estruturas e Fundações, v.1, série A, pt.2, p.9911002.

GADALA, M.S.; DOKAINISH, M.A.; ORAVAS, G.AE. (1984). Formulation methods of geometric and material nonlinearity problems. International Journal for Numerical Methods in Engineering, v.20, p.887-914.

GADALA, M.S.; ORAVAS, G.AE. (1984). Survey of formulation methods and solution techniques. Computers and Structures, v.19, n.5/6, p.865-877.

HEIJER, C.D.; RHEINBOLDT, W.C. (1981). On steplength algorithms for a class of continuation methods. SIAM Journal Numerical Analysis, v.18, n.5, p.925-947.

HINTON, E. (1993). Introduction to nonlinear finite element. Glasgow, UK, NAFEMS.

JEPSON, A.; SPENCE, A. (1985). Folds in solutions of two parameter systems and their calculation: part I. SIAM Journal Numerical Analysis, v.22, n.2, p.347-368.

KAO, R. (1974). A comparison of Newton-Raphson methods and incremental procedures for geometrically nonlinear analysis. Computers and Structures, v.4, p.1091-1097

KANCHI, M.B. (1993). Matrix methods of structural analysis. 2.ed. New York, John Wiley. 
KOUHIA, R.; MIKKOLA, M. (1989). Tracing the equilibrium path beyond simple critical points. International Journal for Numerical Methods in Engineering, v.28, p.2923-2941.

LIPSCHUTZ, S. (1994). Álgebra linear: teoria e problema. 3.ed. São Paulo, Makron Books. (coleção Schaum).

LUSAS (1995). Finite element sytem, version 11. Survey, UK, FEA.

MALERBA, P.G. (1990). Introduzione all analisi di strutture in cemento armato. In: CORSO DI AGGIORNAMENTO SU INTRODUZIONE AL METODO DEGLI ELEMENTI FINITI, Politecnico di Milano, 5-9 nov.

MALLETT, R.H.; MARCAL, V. (1968). Finite element analysis of nonlinear structures. Journal of the Structural Division, v.94, n.ST9, p.2081-2105.

MALVERN, L.E. (1969). Introduction to the mechanics of a continuous medium. Englewood Cliffs, Prentice Hall.

MAZZILLI, C.E.N. (1986). Comportamento não-linear de pórticos planos em regime elástico linear. In: CONGRESSO LATINO-AMERICANO SOBRE MÉTODOS COMPUTACIONAIS EM ENGENHARIA, 7., São Carlos. Anais. v.1, p.381-395

MAZZILLI, C.E.N. (1988). Considerações sobre não-linearidade geométrica em estruturas reticuladas planas. Anais EPUSP, Engenharia Civil, Engenharia de Estruturas e Fundações, v.1, série A. pt.2, p.653-676. 
MAZZILLI, C.E.N. (1996). Some interesting aspects on the elastic instability of simple trusses. In: TAROCO, E.; VENÂNCIO FILHO, F.; BEVILACQUA, L., ed. Recent developments in solid mechanics (Proc. of Symposium to the 70th birthday of Wolf Altman, Rio de Janeiro, 31/07-02/08-1996). Rio de Janeiro, LNCC/ CNPq. p.93-97.

MOORE, G.; SPENCE, A. (1980). The calculation of turning points of nonlinear equations. SIAM Journal Numerical Analysis, v.17, n.4, p.567-576.

NOGUCHI, H.; HISADA, T. (1995). Integrated FEM formutation for total/ updated lagrangian method in geometrically nonlinear problems. JSME International Journal, v.38, série A, n.1, p.23-29.

OÑATE, E.; MATIAS, W.T. (1996). A critical displacement approach for predicting structural instability. Computer Methods in Applied Mechanics and Engineering, v.134, p.135-161.

ORAN, C. (1973). Tangent stiffiness in plane frames. Journal of the Structural Division, v.99, n.ST6, p. 973-985.

PETERSON, A.; PETERSSON, H. (1985). On finite element analysis of geometrically nonlinear problems. Computer Methods in Applied Mechanics and Engineering, v.51, p.277-286.

PIMENTA, P.M. (1986). Aspectos da análise não-linear de estruturas reticuladas. In: CONGRESSO LATINO-AMERICANO SOBRE MÉTODOS COMPUTACIONAIS EM ENGENHARIA, 7., São Carlos. Anais. v.1, p.449-464

PIMENTA, P.M. (1988a). Análise não-linear de pórticos planos. Anais EPUSP, Engenharia Civil, Engenharia de Estruturas e Fundações, v.1, série A, pt.1, p.561-582. 
PIMENTA, P.M. (1988b). A matriz de rigidez tangente do elemento de pórtico plano: teoria de Timoshenko. Anais EPUSP, Engenharia Civil, Engenharia de Estruturas e Fundações, v.1, série B, pt.1, p.1027-1034.

PIMENTA, P.M. (1988c). Um método geral para a dedução da matriz de rigidez tangente de elementos finitos. Anais EPUSP, Engenharia Civil, Engenharia de Estruturas e Fundações, v.1, série B, pt.1, p.1005-1023.

RAM, E. (1981). Strategies for tracing the nonlinear response near limit points. In: WUNDERLICH, W.; STEIN, E.; BATHE, K.-J, eds. Nonlinear finite element analysis in structural mechanics. Berlin, Springer-Verlag.

RHEINBOLDT, W.C. (1981). Numerical analysis of continuation methods for nonlinear structural problems. Computers and Structures, v.13, p.103-113.

RHEINBOLDT, W.C. (1978). Numerical methods for a class of finite dimensional bifurcation problems. SIAM Journal Numerical Analysis, v.15, n.1, p.1-11.

RIKS, E. (1972). The application of Newton's method to the problem of elastic stability. Journal Applied Mechanics, v.39, p. 1060-1066.

RIKS, E. (1978). An incremental approach to the solution of snapping and buckling problems. International Journal Solids Structures, v.15, p.529-551.

RIKS, E. (1978). Some computational aspects of the stability analysis of nonlinear structures. Computer Methods in Applied Mechanics and Engineering, v.47, p.219-259. 
RUBERT, J.B. (1993). Estudo do desempenho de algoritmos numéricos na solução de sistemas não-lineares de estruturas formadas por barras de treliça. São Carlos. Dissertação (Mestrado) - Escola de Engenharia de São Carlos, Universidade de São Paulo.

SCHWEIZERHOF, K.H.; WRIGGERS, P. (1986). Consistent linearization for path following methods in nonlinear FE analysis. Computer Methods in Applied Mechanics and Engineering, v.59, p.261-279.

SIMO, J.C.; WRIGGERS, P.(1990). A general procedure for the direct computation of turning and bifurcation points. International Journal for Numerical Methods in Engineering, v.30, p.155-176.

SIMPSON, R.B. (1975). A method for the numerical determination of bifurcation states of nonlinear systems of equations. SIAM Journal Numerical Analysis, v.12, n.3, p.439-451.

SOUZA LIMA, V.M.; VENÂNCIO FILHO, F. (1982). Considerações sobre a não-linearidade geométrica de estruturas reticuladas. in: ESCOLA DE MATEMÁTICA APLICADA, 3., Laboratório de Computação Científica (CNPq), Rio de Janeiro. p1-38.

SOUZA LIMA, V.M.; VENÂNCIO FILHO, F. (1984). A noção de rigidez tangente no estudo da não-linearidade geométrica de estruturas reticuladas. In: CONGRESSO LATINO-AMERICANO SOBRE MÉTODOS COMPUTACIONAIS EM ENGENHARIA, 5., Salvador. p.1-24

STAKGOLD, I. (1971). Branching of solutions of nonlinear equations. SIAM Review, v.13, n.3, p.289-332. 
SZILARD, R. (1985). Critical load and post-buckling analysis by FEM using energy balancing technique. Computers and Structures, v.20, n.1-3, p.277-286.

SZILARD, R. (1986). An energy balancing strategy for solution of combined geometrical and material nonlinearity problems. Computers and Structures, v.23, n.2, p.147-162.

UNIVERSIDADE DE SÃO PAULO. Escola de Engenharia de São Carlos. Serviço de Biblioteca (1997). Diretrizes para elaboração de dissertações e teses na EESC-USP. 2.ed. São Carlos. 58p.

ZIENKIEWICZ, O.C.; TAYLOR, R.L. (1989). The finite element method. 4.ed. London, McGraw-Hill.

YAGHMAI, S.; POPOV, E.P. (1971). Incremental analysis of large deflections of shells of revolution. International Journal Solids Structures, v.7, p.1375-1393.

WASZCZYSZYN, Z. (1983). Numerical problems of nonlinear stability analysis of elastic structures. Computers and Structures, v.17, n.1, p.13-24.

WEINITSCHKE, H.J. (1985). On the calculation of limit and bifurcation points in stability problems of elastic shells. International Journal Solids Structures, v.21, n.4, p.79-95.

WEN, R.K.; RAHIMZADEH, J. (1983). Nonlinear elastic frame analysis by finite element. Journal of Structural Engineering. v.109, n.8, p.19521971. 
WERNER, B.; SPENCE, A. (1984). The computation of symetriy-breaking bifurcation points. SIAM Journal Numerical Analysis, v.21, n.2, p.388399.

WONG, M.B.; TIN-LOI, F. (1990). Geometrically nonlinear analysis of elastic framed structures. Computers and Structures, v.34, n.4, p.633640 .

WRIGGERS, P.; WAGNER, W., MIEHE, C. (1988). A quadratically convergent procedure for the calculation of stability points in finite element analysis. Computer Methods in Applied Mechanics and Engineering, v.70, p.329-347.

WRIGGERS, P. (1990). Lectures on continuum mechanics, nonlinear finite element techniques and computacional stability. Udine, Italy, CISM. (Notas do curso de análise não-linear). 


\section{ANEXO A}

\section{ELEMENTOS DE ANÁLISE FUNCIONAL NECESSÁRIOS PARA A DEFINIÇÃO DE CRITÉRIOS PARA CARACTERIZAÇÃO DE PONTOS SINGULARES}

O sistema de equações (4.17) resulta de uma aproximação por elementos finitos e, portanto, gera soluções que pertencem a um espaço de dimensão finita. Além disso, espera-se que esta sequência de soluções aproximadas, em decorrência de refinamentos da discretização, tenha propriedades de convergência para a solução exata do modelo matemático.

De um ponto de vista matemático, as soluções aproximadas com as características acima são elementos de um espaço vetorial normado (em particular com produto interno) de Banach (no qual toda sequência de soluções é convergente).

Num tratamento mais formal, considerem-se primeiramente dois espaços de Banach X e Y de dimensão finita, esquematizados na figura 1, e admita-se que haja uma transformação linear $\mathrm{T}$ que leva elementos de $\mathrm{X}$ a elementos em $Y$ ou seja: para qualquer $x \in X, y=T x \in Y$. Em seguida, considerem-se outros dois espaços de Banach $X^{\star}$ e $Y^{\star}$, duais de $X$ e $Y$, respectivamente, e $T^{\star}$ um operador linear dual que leva elementos de $Y^{*}$ a elementos em $\mathrm{X}^{*}$, tal que: $\mathrm{x}^{*}=T^{*} \mathrm{y}^{*}$, qualquer $\mathrm{y}^{*} \in \mathrm{Y}^{*}$. 
Os produtos de dualidade entre elementos dos espaços $X$ e $Y$ com elementos dos seus respectivos espaços duais, são representados genericamente por $\left\langle x, x^{*}\right\rangle$, sendo $x \in x^{*}$, respectivamente, elementos dos espaços primal e dual.

Isto posto, o operador $\mathrm{T}^{\star}$ é definido como adjunto se a seguinte propriedade é verificada: $\left\langle x, T^{*} y^{*}\right\rangle=\left\langle T x, y^{*}\right\rangle$ para todo $x \in X, x^{*} \in X^{*}, y \in$ $Y$ e $y^{*} \in Y^{*}$, ver figura A.1.

Deste modo, considerando-se que $y=T x$ e $x^{*}=T^{*} y^{*}$, tem-se:

$$
\left\langle x, x^{*}\right\rangle=\left\langle y, y^{*}\right\rangle
$$

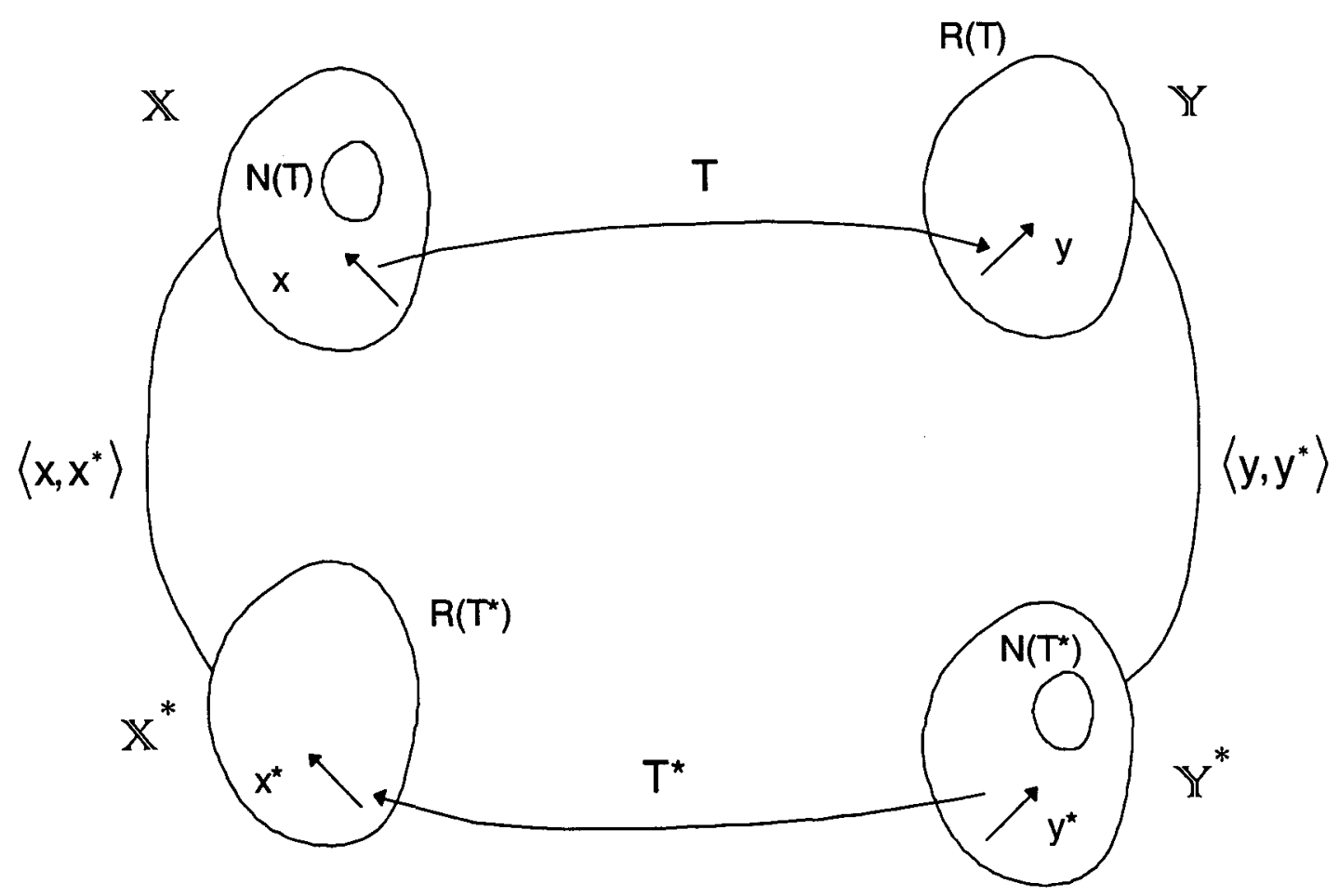


Define-se como núcleo da aplicação linear $T$ ○ subespaço de $X$ formado pelos seus elementos que são levados ao vetor nulo de Y. Admitese neste estudo, que o núcleo da transformação $T$, representado por $N(T)$, é gerado por um subespaço de $m$ vetores linearmente independentes de módulo unitário. Admite-se, também, que a imagem de $T, R(T)$, é um subespaço vetorial de dimensão $m$.

Também a transformação adjunta tem um núcleo $\mathrm{N}\left(\mathrm{T}^{\star}\right)$ e uma imagem $R\left(T^{\star}\right)$, ambos de dimensão $m$. Note-se ainda que o conjunto de vetores $\mathrm{x}^{*} \in \mathrm{X}^{*}$ tais que,$\left\langle\mathrm{x}, \mathrm{x}^{*}\right\rangle=0$ para $\forall \mathrm{x} \in \mathrm{X}$, pertence ao complemento ortogonal de $\mathrm{X}$, representado por $\mathrm{X}^{\perp}$. Valem, em consequência, as seguintes propriedades:

$$
\begin{aligned}
& N(T)=R\left(T^{*}\right)^{\perp} \\
& R(T)=N\left(T^{*}\right)^{\perp} \\
& N\left(T^{*}\right)=R(T)^{\perp} \\
& R\left(T^{*}\right)=N(T)^{\perp}
\end{aligned}
$$

De fato, por exemplo, seja $v \in N(T)$ então $T v=0$. Assim, é possivel tomar um elemento qualquer de $\mathrm{X}^{\star}$ e operar o produto de dualidade com TV. Desse modo, $\left\langle x^{*}, T v\right\rangle=0$, mas considerando-se o operador adjunto $\left\langle x^{*}, T v\right\rangle=\left\langle T^{*} x^{*}, v\right\rangle$ e então $\left\langle T^{*} x^{*}, v\right\rangle=0$. Como $T^{*} x^{*} \in R\left(T^{*}\right)$, logo $v \in R\left(T^{*}\right)^{\perp}$, de onde resulta a primeira propriedade.

As outras propriedades demonstram-se de maneira análoga.

Para efeito de exemplificação, na Mecânica do Contínuo, do operador adjunto considere-se que os espaços $\mathrm{X}, \mathrm{Y}, \mathrm{X}^{*}$ e $\mathrm{Y}^{*}$ sejam respectivamente 
os espaços das tensões, das forças, das deformações $e$ dos deslocamentos. Pode-se observar que a relação entre tensões e forças é feita por equações de equilíbrio e entre deslocamentos e deformações por equações de compatibilidade.

Assim, os elementos do espaço das deformações são colocados em correspondência com os elementos do espaço dos deslocamentos através de uma transformação linear $\mathrm{T}$.

Os espaços das forças e das tensões são duais aos dos deslocamentos e de deformações, respectivamente, e são também espaços de Banach. Por definição, a transformação linear $T^{*}$ associa elementos do espaço das tensões com elementos do espaço vetorial das forças. Como se mostrará em seguida, $T^{*}$ é denominada transformação adjunta e no caso particular da Mecânica ela coincide com a transposta de $T$.

A figura A.2 mostra num plano três pontos vizinhos, $P, Q$ e $R$ nas posições indeformada e deformada. Admitem-se deformações e deslocamentos pequenos.

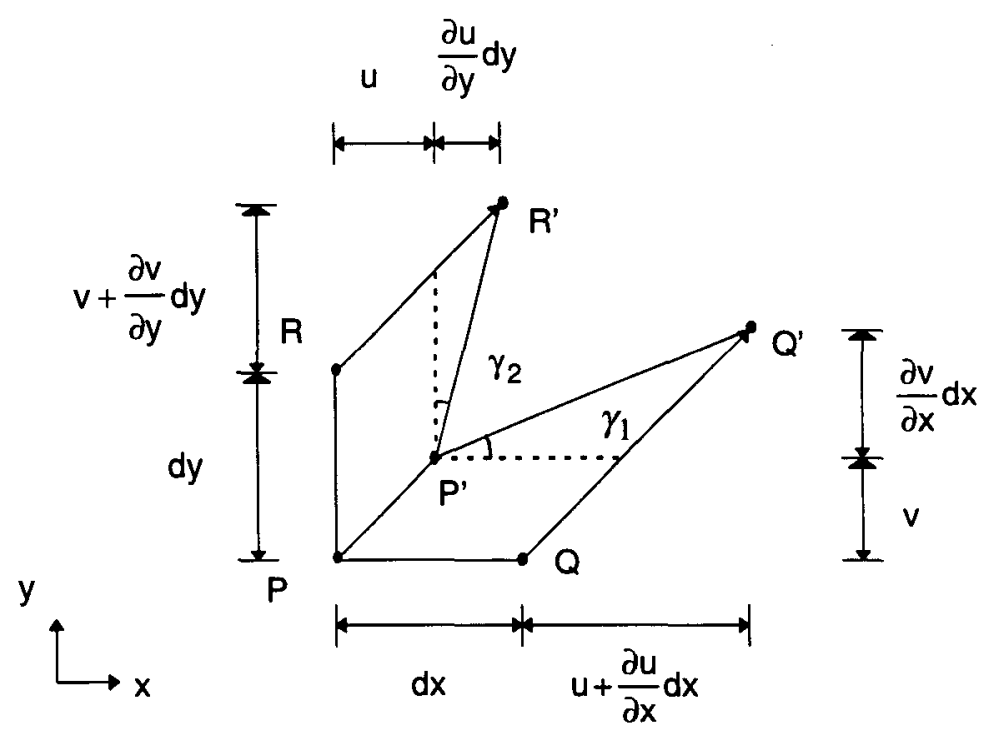

FIGURA A.2- Posição inicial e deslocada de três pontos próximos 
Assim, por meio de equações de compatibilidade tem-se:

$$
\begin{aligned}
& \varepsilon_{x}=\frac{\partial u}{\partial x} \\
& \varepsilon_{y}=\frac{\partial v}{\partial y} \\
& \gamma_{x y}=\frac{\partial v}{\partial x}+\frac{\partial u}{\partial y}
\end{aligned}
$$

ou matricialmente:

$$
\left[\begin{array}{cc}
\frac{\partial}{\partial x} & 0 \\
\frac{\partial}{\partial y} & \frac{\partial}{\partial x} \\
0 & \frac{\partial}{\partial y}
\end{array}\right]\left\{\begin{array}{l}
u \\
v
\end{array}\right\}=\left\{\begin{array}{c}
\varepsilon_{x} \\
\gamma_{x y} \\
\varepsilon_{y}
\end{array}\right\}
$$

Logo, neste caso, o operador T é dado por:

$$
T=\left[\begin{array}{cc}
\frac{\partial}{\partial x} & 0 \\
\frac{\partial}{\partial y} & \frac{\partial}{\partial x} \\
0 & \frac{\partial}{\partial y}
\end{array}\right]
$$

A figura A.3 mostra um elemento infinitesimal, de lados $d x$ e dy, sujeito ao estado plano de tensão, cujas componentes estão representadas em cada face do elemento. A força por unidade de volume apresenta componentes $f_{1} \in f_{2}$, respectivamente, nas direções $x \in y$. 


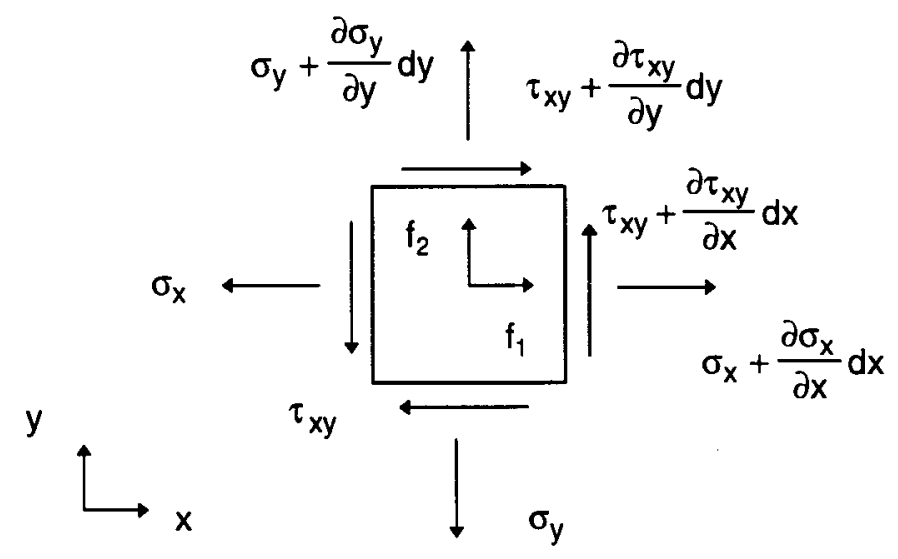

FIGURA A.3- Diferenciais de tensão em equilíbrio

Assim, par0a o caso mostrado na figura A.3 utilizando-se equações de equilíbrio tem-se:

$$
\begin{aligned}
& \frac{\partial \sigma_{x}}{\partial x}+\frac{\partial \tau_{x y}}{\partial y}+f_{1}=0 \\
& \frac{\partial \tau_{x y}}{\partial x}+\frac{\partial \sigma_{y}}{\partial y}+f_{2}=0
\end{aligned}
$$

ou matricialmente:

$$
\left[\begin{array}{ccc}
\frac{\partial}{\partial x} & \frac{\partial}{\partial y} & 0 \\
0 & \frac{\partial}{\partial x} & \frac{\partial}{\partial y}
\end{array}\right]\left\{\begin{array}{l}
\sigma_{x} \\
\tau_{x y} \\
\sigma_{y}
\end{array}\right\}=\left\{\begin{array}{l}
-f_{1} \\
-f_{2}
\end{array}\right\}
$$

Neste caso o operador $T^{*}$ é dado por:

$$
T^{*}=\left[\begin{array}{ccc}
\frac{\partial}{\partial x} & \frac{\partial}{\partial y} & 0 \\
0 & \frac{\partial}{\partial x} & \frac{\partial}{\partial y}
\end{array}\right]=T^{\top}
$$

